\title{
A VLSI DESIGN FOR AN EFFICEENT MULTIPROCESSOR CACHE MEMORY
}

\section{CENTRE FOR NEWFOUNDLAND STUDIES}

\section{TOTAL OF 10 PAGES ONLY MAY BE XEROXED}

(Without Author's Permission) 




\section{A VJSI DESIGN FOR AN EFFICIENT}

\section{MULTIPROCESSOR CACHE MEMORY}

BY

i.

Nino Luo

A thesis

submithed to the School of Graduate Studlies

in partial fulfillment of the recyuirements for

the degree of Master of Science

Department of Computer Science

Ninerial l'uiversity of Newfondland

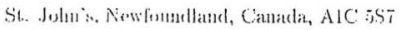

Xus..Hin. $\angle 8,1980$ 
The author has granted an irrevocable nonexclusive licence allowing the National Library of Canada to reproduce, loan, distribute or sell copies of his/her thesis by any means and in any form or format, making this thesis available to interested persons.

The author retains ownership of the copyright in his/her thesis. Neither the thesis nor substantial extracts from it may be printed or otherwise rerroduced without his/her permission.
L'auteur a accordé une licence irrévocable et non exclusive permettant à la Bibliothèque nationale du Canada de reproduire, prêter, distribuer ou vendre des copies de sa thèse de quelque manière et sous quelque forme que ce soit pour mettre des exemplaires de cette thèse à la disposition des personnes intéressées.

L'auteur conserve la propriété du droit d'auteur qui protège sa thèse. Ni la thèse ni des exiraits substantiels de celle-ci ne doivent être imprimés ou autrement reproduits sans son autorisation. 


\begin{abstract}
This thesis proposes a cache memory, uscd for a 32-bit processor systen, which consists of four components: the Dircetory, Line Replacement Unit (LRU), Gache Menory, and Control Unit. An 8-way set-associative mapping method is cmployed in the directory. The Line Replacement Unit is based on the least recently used line replacement algorithm. The cache memory unit has a capacity of $8 \mathrm{k}$ bytes, 32 bytes in each line, and it is directly accessible to $1,2,3$, or 4 bytes (one word) once by the associated processor. This cache menory is designed for a multiple processor system as well as in single processor system; a write-through algorithm and an updating algorithm are combined together to kecp the information in main memory consistent with that of the cache and to make the multicaches coherent. The hit ratios are predicted to be over 95 percent. A two-phase clock of 40 ns is employed to pipeline this cache, and it can turn out a result in 20ns during read operations without line misses. This cache is inıplemented into a single chip, and is designed so that it is possible to build cache systems of varioue sizes using these chips, without decreasing the system speed. This caclie memory has been laid ont as a single integrated circuit using 3 Micron NTCMOS teclınology, and its electrical and logical behavior has been simulated.
\end{abstract}




\section{Acknowledgments}

First of all, I would like to express my sincere gratitude to my advisor Dr. Paul Gillard for his supervision, guidance, suggestions, encouragement, and patience, which have greatly helped me to complete this thesis.

I would like to thank Dr. Wlodek Zuberek, who has contributed his support nnd advice townrd the developinent of this thesis, and the other staff membors of Department of Computer Science, who have provided me with their technical support. Especially the support and patience of my wife was of great help.

I an very grateful to Department of Compuler Science and School of Graduate Studies, Memorial University of Newfoundland, for providing me with an opportunity for graduate studies and financial support in the form of a fellowship and tcaching assisl.antship during my study. 
To the Memory of My Father and to My Mother 


\section{Contents}

1 INTRODUCTION 1

2 BASICS OF CACHE MEMORY 6

2.1 Overview of the Memory llierarchy ............ 7

2.2 The Concept of Cache Menory ... . . . . . . . . . . 8

2.3 The Basic Structure of Cache Mernory ... . . . . . . . . 10

2.4 The bine Size Choice ................... I2

2.5 A Survey of Cache Design ... . . . . . . . . . 16

3 IMPLEMENTATION OF THE CACHE ALGORITHMS 22

3.1 Gache Design Yaraneters . . . . . . . . . . . . . . . 22

3.2 The Structure of the Cache Memory ... . . . . . . . . 31

3.3 The Address Space Mapping . . . . . . . . . . . . . . . 34

3.4 The Set-associative Mapping . . . . . . . . . . . . . . 36

3.5 Implementation of the Directory . . . . . . . . . . 37

3.5.1 The Line Slot of the Directory .............. 40

3.5.2 The Address Register . . . . . . . . . . . . . . 45

3.5.3 The 32-bit Decoder for Set Selection ... . . . . . . . 47

3.5.4 The Line Number Generator . . . . . . . . . . . . . . . 49

3.6 The Line Replacement Unit . . . . . . . . . . . . . . 55

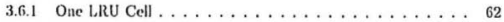

3.6.2 One Bit of the LRU Cell . . . . . . . . . . . 65 
4 THE MEMORY AND CONTROL UNITS

4.1 Structure of the Memory . . . . . . . . . . . . . . 70

4.1.1 The Cacle Memory Register . . . . . . . . . . 72

4.1.2 The 256-bit Row Decoder . . . . . . . . . . . . . 80

4.1.3 The Cache Memory . . . . . . . . . . . . . . . . 811

4.1 .1 The Data Bus Control Circuit . . . . . . . . . . . . . 81

4.2 The System Control Unit. . . . . . . . . . . . . . . 94

4.2.1 The Regular Read/Write Operations .......... M

4.2 .2 The Update Operations $\ldots \ldots \ldots \ldots \ldots$

4.2 .3 The Miss Operations . . . . . . . . . . . . 142

5 USE IN A MULTIPROCESSOR SYSTEM 111

5.1 The Coherence Solution Strategy . . . . . . . . . . . 111

5.1.1 The Protocols between the Bus and the Cache . . . . . . . 117

5.1.2 The Protocols between the Processot and the Cathe . . . . I22

5.2 External Interface . . . . . . . . . . . . . . 126

5.2 .1 The Interface Signals . . . . . . . . . . . . I26i

5.2 .2 The Timing Operations . . . . . . . . . . . . I:30

5.3 Consideration of the System Bus and Main Memory . . . . . . . 135i

5.4 Simulations of the Cache-based Multiprocessor . . . . . . . . . . 1399

5.5 Testing the Cache Menory . . . . . . . . . . . . . A

$\begin{array}{lll}6 & \text { CONCLUSIONS } & 147\end{array}$ 


\section{List of Figures}

$1 \wedge$ Von Neumanu Computer Organization . . . . . . . . . . 2

2 ^ Typical Menory Hicrarchy ............... 8

3 The Basic Cache Menory Structure . . . . . . . . . . . . . 32

4 The Sel-issociative Mapping . . . . . . . . . . 35

5 The Directory $\ldots \ldots \ldots \ldots \ldots \ldots \ldots \ldots$

6 Simelation of the Tag Aray ................ 41

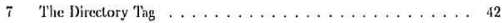

8 'The Tag Bit and Valid Bit of the Directory . . . . . . . . . . 12

9 One Bit of the Address Register . . . . . . . . . . . . . . 46

10 The D-type Rising-edge-triggered Flip-flop . . . . . . . . . . 46

11 The 32 -bit Directory Decoder ............... di

12 The 16-bit Decoder .................... 48

13 Simulation of the 32-bit Decoder ............. 50

14 The Line Number Generator . . . . . . . . . . . . 51

15 Layout of the Line Number Generator . . . . . . . . . . 52

16 Simulation of the Line Number Generator ... . . . . . . . 53

17 Structure of the LRU Unit . . . . . . . . . . . . . . 56

18 The One-shot Circuit . . . . . . . . . . . . . 6 60

19 Structure of an LRU Cell . . . . . . . . . . . . . 6 63

20 An Exanple of the LRU Algorithı . . . . . . . . . . . 64 
21 Layout of the LRU Cell ................... 66

22 One Bit of the LRU Cell . . . . . . . . . . . . 67

23 Simulation of the LRU Unit . . . . . . . . . . 68

24 Structure of the Cache Memory ............. it

25 One Bit of the Memory Register . . . . . . . . . . . 72

26 The Register/Counter Logical Circuit.............. it

27 Layout of the Mernory Colurnn Control . . . . . . . . . . . 76

28 Simulation of the Memory Column Control . . . . . . . . . . 77

29 Circuit of the Transfer Decomposer . . . . . . . . . 78

30 The 256 -bit Memory Decoder . . . . . . . . . . . . 79

31 Simulation of the 256-bit Row Decoder . . . . . . . . . 81

32 The Memory Arrays ................... 82

33 Four Bits of the Mernory . . . . . . . . . . . 83

34 The Dala Bus Control Circuit ................. 84

35 One Bit of the Gate Circuit . . . . . . . . . 85

36 The Gate Control Logic . . . . . . . . . . . . . . 88

37 Layout of the Gate Logic . . . . . . . . . . . . . . . 89

38 Simulation of the Gate Logic . . . . . . . . . . . . . 90

39 The Bus Write/Read Operation Control ............ 91

40 Simulation of the Writc Control . . . . . . . . . . . . 92

41 Sinulation of the Read Control .............. 93

42 The Single Following Pulse Producer . . . . . . . . . . 95 
43 The Clock Pulse Generator . . . . . . . . . . . . . . . 96

14 A Timing Diagram for the Clock Pulse Generator ......... 996

15 The Circuit of the Bus Update Watcher . . . . . . . . . . . . 99

16 the Update-Write Generator . . . . . . . . . . . . . 102

17 The Miss Circuit . . . . . . . . . . . . . . . . 102

18 The Circuit for the Bus Control Sigual Generator . . . . . . . 105

19 The Update Request Clear Circuit ... . . . . . . . . 107

50 The Read Valid Circuit for Read Miss . . . . . . . . . . . . 109

51 A 'lypical Cache-based Multiprocessor System . . . . . . . . . 112

52 Communication between the Cache and Bus for a Write Operation. I18

53 Communication between the Cache and Bus for a Line Miss ... . 120

54 Conmunication for an Update Opcration . . . . . . . . . . 121

55 The Processor Sulsystem . . . . . . . . . . . . . . . . . . 124

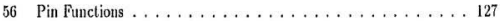

57 A Tiuning Diagram for Read/Write Operations . . . . . . . . . 131

58 A 'Timing Diagram for Read Operations with a Line Miss . . . . . 132

59 A Timing Diagram for Write Opcrations with a Line Miss . . . . I33

60 The $\mathrm{N}$-User 1 -Server Bus Arbiter . . . . . . . . . . . 136

61 'llie Arbitration Unit . . . . . . . . . . . . . . . . . 137

62 The Shared Main Mennory Partitioned into 8 Modules . . . . . . . 139

63 Simulations for the Multiprocessor System . . . . . . . . . . 143

64 The Testing Circuil for Shifling-out Cache Line Addresses . . . . 146 


\section{List of Tables}

1 The Design Target Miss Ratios . . . . . . . . . . If

2 The Relevant Cache-mapping-type Ratio . . . . . . . . 15

3 The Truth Table Relating the "Hot Code" and the Binary Code . . 54

4 Time Delay for the Directory $\ldots \ldots \ldots \ldots \ldots \ldots \ldots$

5 The Gate Control Functions . . . . . . . . . . 85 


\section{INTRODUCTION}

In 1945, Jolu Von Neumann made proposals for a digital electronic computer structure. In his proposals, the basic logical structure of a digital computer system has the following characteristics:

1. It has ant input medium, by meatns of which an csscntially unlimitod number of operands or instructions may be entered.

2. It has stornge, from which operands or instructions maty be obtained and into which result $\mathrm{s}$ may be entercd, in any desired order.

3. It has a calculating unil, capable of carrying out arithmetic and logical operations on any operands taken from storage.

4. It has an output medium, by means of which an essentially unlinited number of results may be delivered to the users.

5. It has a control unit, capable of interpreting instructions obtained from memory or storage, and capable of clioosing between alternate courses of action on the basis of computed results.

In general, a computer which meets the criteria defined as the Von Neumann structure is organisod as shown in Fig. 1. Although the components of the five parts of the hasic struct ure and the technologies used may vary wideiy, the functions of the parts nuing be clearly identified in virtualiy any digital computer. 


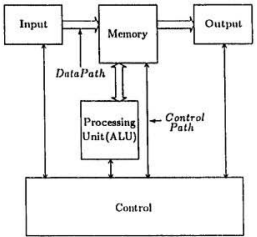

Figure 1: A Von Neumann Computer Organization

Memory is the source of all information, data, and instructions, llowing lo ur from the four other parts. The data and instructions are stored in the memory cells. each of which is associated with a location, or address. The colls can be acensert by other parts of the computer by means of these addresses.

The main functions of input and output, as indicated by therit nams. are lo derive information from and to deliver the results and oflher inlormil iun tw Ih.. outside world. They have also two subsidiary functions, buffering atur flatin con version. The buffering function provides an interface and synchurouizatiun lu-twex+11 the processing part of the computer and the ontside world. The consersion funt:tion can convert the data type in the processing unit into forms userl untsirle the computer system. 
The processing part of a computer, referred to as the arithmetic-logic unit, implements the various aritlımetic and logic operations on operands obtained from the memory. The results, after these operations, are lypically stored back in the memory.

The control unit obtains instructions from the memory, decodes them, and, depending on their meaning, sends the appropiate control signals to other parts of the conputer so that the desired operations will be accornplished. It also makes decisions atbout what action must be taken after receiving the results of various tests on date made by the arithmetic-loyic unit. The combination of the arithnel ic-logic unit and control unit is known as the central processing unit, or processing elenent in the case of multiple processor systems.

Until the last two decades, alnost all the electronic digital computer systems used this Von Neumann architecture. Even when the underlying architectures of the computer systems began to contain a limited amount of parallelism (such as in the CDC6600, for example) it was generally concealed from the users. In this period, the demand for higher specd, larger storage, and roore reliable computer systems wis rapidly increasing because large scale computation applications were vistalized. The dentand was such that, despite many technological advances in clectronics, uniprocessor systcms proved to be inadequate for the most highly computationally intensive problems sitce the point had been reached where conmunication delays betwecn switching elements or integrated circuits play it dominant role in the speed of the computation. Therefore, new ways had to be found to meet 
these requirements. The general approach is based on parallelism, inplying that computer architectures will have to depart from the strict Von Neumann concept. Parallelism in various forms had already appeared in computers produced during the 1960's, and has proved to be an effective approach. In this context, parallelism docs not only mean the replication of logic but also has other neanings, for example, a uniprocessor using a pipelined instruction unit and a pipelined arillmetic unit, as well as the implementation of multiple programs excculed "simultancously", all imply concepts of parallelism. 'Therefore parallelism in a computer systern presently has three meanings:

1. Time interleaving

\section{Resource replication}

\section{Resource sharing}

Time itterleaving introduces a time factor into the concept of parallelism. That is, several process steps are interleaved in time, each using a part of the same hardware at different times. In this case, it is not necessary to have a replication of hardware to increase the performance of a computer system. Pipclining is an example of time-interleaving.

Resource replication is the replication or ardition of leardware units which can operate simultancously on a problem, thereby attaining computation power through replication of logic, rather than relying soldy on fast individhal gittes and 
small dimensions to reduce logic delay in order to oblain high speed. Multiple processes using the same hardware in some time-slice order are an cxample of resource siraring.

Since parallelisu was introduced into compuler architecture, various parallel computer architectures such as vector processors, pipelines, array processors, as well as multiprocessor archilectures, have been developed and used to handle large quantitics of data simultaneously and concurrently with high performance. $1 / 0$ processors have heen used for input and outpul to sperd tip conmunication betwecn the processing elements and extemal stornge or users. 'Thus, in general, parallelism includes not only simultaneity but also concurrency. The fomer means that two or more events occur at the same time and the latter means that two or more events occur tvithin a given interval of time.

On the other hand, memory has been organized in different ways in order to obtain access specds compatible with that of processing elements and to have a larger capacity. In gencral, there are two basic approachcs: one is to organize the menory as a menory liearchy; the other is to decompose the memory into several modules shared by the processors in the system.

These kinds of computer architectures are, more or less, not strictly Vou Neumaun structures; indeed, the multiple processor systems in pew icular have quite different characteristics. 


\section{BASICS OF CACHE MEMORY}

Throughout the history of electronic computers, whenever developments have taken place in computer systems which increase processor speed, there is corresponding pressure to have the memory match this speed and, at the same time, increase its capacity. Therefore, performance improvenents in computers have been associatcd with improvenents in memory capacity and specd. Although both processors and main memory systems have been improved by steadily developing tecluologies and novel architectures, there has been a persistent mismatch between the speed of processors and that of main memory. That is, the main menory is slow relative to the processors. The memory system limits the speed at which input data can be delivered to a processor and the results received from the processor. This is the socalled Von Neunann bottleneck. llence there has been a constant need for steady improvements to main memory subsystems for high overall system pcrformance. Approaches of interest toward improving memory speed and capacity have been the following $[2,5,7]$ :

1. Memory hierarchies and virtual memory

2. Cache memories

3. Devclopment of larger and faster memory chips

4. Memory interleaving 


\subsection{Overview of the Memory Hierarchy}

In order to improve the performance of computer systems, especially single processor systems, there are two approaches to speed up a memory system with a large capacity. One is to develop a higiner speed memory system with a larger capacity, the other is to partition a memory system into an efficient memory hierarchy consisting of several levels of subsystens with various speeds and sizes $[2,3,5]$. The first approach scems more straightforward and simple - to have a fast one-level memory with a large capacily. However, even with improvenents in tednology, a fast menory system with a large capacity is still very expensive, so that it is necessary to use slower memory at a lower cost to create a memory system with a large enongh capacity. In order to give the memory subsystem an adequate effective spced, the memory subsystem can be organized as a hierarchicnl memory system. This kind of memory system can be matched to both the speed and size requirements of the high-speed processor at relatively low cost. $\wedge$ typical hierarchical menory structure is depicted in Pig 2. The top level of the menory hierarchy (near the processor) has the fastest speed but also the highest cost. Therefore, the capacity of this level is made smaller to decrease system costs. For the lower levels, the spect of the subsystem decrenses while the capacily increases. At the level on the bottom of the memory hierarchy, the memory subsystem possesses the largest capacity, but slowest speed, with lowest cost per word stored. In this memory hierarchy, each level is directly connected to the immediately higher level. That is, each memory subsystem can directly communicate with the immediately higher or lower 


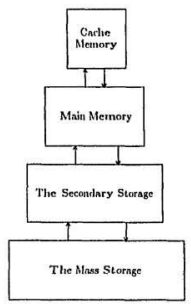

Figure 2: $\Lambda$ Typical Menory Hierarchy

subsystem in the hicrarchy. For example, the pucessors can directly communicate with the first-level menory, e.g. register array or cache memory; and sinilarly the first-level subsystem can communicatc with the sccond-level onc, as shown in Fig. 2, and so on. Generally the top-level subsystem, such as cache memory, is used to attempt to bridge the speed gap between the processors and the lower level subsystem, while the lower level memory subsystems are employed to cularge the capacity of the whole memory system.

\subsection{The Concept of Cache Memory}

The concept of cache memory was proposed by Wilke [1965] in a brief article in which he described a system that contained two kinds of main menory: one was conventional, and the other was unconventional high-speed menory called at that. 
time slave memory, now called cache memory. In 1968, the first real cache memory was implemented on the IBM $360 / 85$. Since then, use of cache memory has rapidly increased on a wide range of computer systems, initially on mainframes, then on minicomputers, and today even on microcomputers.

Cache memory, a relatively small, high speed random access memofy, is designed for transparently bridging the speed gap between the $\mathrm{CPU}$ and main menory, since it typically has a speed compatible with that of the CPU. This means that a cache metnory in a cache-based system is invisible and not directly accessible to users or even to system operators. Typically, the speed of caclie memory is five to ten times faster than that of main momory. Using this kind of memory hierarchy, the computer may seem to have a one-level memory with the capacity of the slow main memory and the speed of the cache memory [2].

The idea of the cache memory, similar to the primary-secondary virtual memory, is to duplicate the active portions of a lower speed memory in a high speed, but smaller, memory. Only the data most likely to be needed in near future by the CPU reside in the cache, and obsolete data are automalically replaced by the newly requested data. In general, the speed of the cache memory is matched to the maximum data rate of the processor so that the processor can access data in the caclie without delay, whenever the data requested by the processor are found in the cache. If the requested data are not in the cache, a cache niss occurs, and a request is made to the main memory for transfer of the requested data to the cache. If the data currently resides in the main memory, it is transferred to 
the cache immediately. If it is not, but is in the secondary memory, a request is issued to bring the requested data from the backing storage. Therefore, when the required references to the memory can be captured by the cache, speed is tot degraded. Otherwise, the performance will be degraded by the time required to transfer data from the main memory to the cache.

The use of cache memories in modern computer systems is based on the locality of memory references - both spatial and temporal $[7,9]$. Spatial locality refers to the property that memory accesses over a short period of time tend to be clustered in space. This type of behavior can be expected based on the common knowledge of typical program behavior: related data items (variables, arrays, etc.) are usually stored together and instructions are mostly executed sequentially. Tenporal locality refers to the property that references to a given locality are lypically clustered in time. This type of behavior can be expected from program loops in which both data and instructions are reused. Therefore, use of a cache memory in a computer system can minimize the interconnection network traffic between the processor and main memory and speed up the system since the access delay of the menory system and the frequency of references to the slower main memory are highly reduced.

\subsection{The Basic Structure of Cache Memory}

The capacity of cache memory is far smaller than that of main memory; that is, the address space of cache memory is far smaller than the address space of main memory, therefore cache memory requires an address mapping mechanism 
to translate the main memory addresses, at a high speed, into the cache memory address where the copies of data in the main memory reside. Also because the most active portions in the main menory are copied in the cache memory, if the caclic memory is full and the associated processor necds data not in the cache memory, sone of the data in the cache will be replaced with the newly requested data from the main memory. There must exist an algorithm which can predict that the data to be replaced will not be used in near future. Since the speed of the cache memory is the key factor in cnelie menory design, this kind of algorithun must be inplemented in hardware. Hence, the basic structure of a cache memory should have at lcast three basic hardware components: an address mapping mechanism, a data replacement unit, and storage for the data in the cache.

The basic functions of a cache memory can generally be described as follows: Each reference from the processor to a memory location is presented to the cache memory. The cache first scarclics the directory of the address mapping mechanism to sce if the requested data reside in the cache memory. If the requested datia are in the cache, the data are operated on to satisfy the processor immediately without disturbing the main memory. If the data are not resident in the caclse, a cache miss occurs which will cause the transfer of the new data from the main memory to the cache. 'Then the requested data can be referenced by the processor. Before transferring a new line to the cache, some data lias to be removed from the caclie menory to make room for the new. Which old data in the cache will be discardect is determined by the data roplacement unit. 'Therefore, the cache-replacement de. 
cision directly affects the performance of the cadie. A good replacement algorithm can make the cache have a somewhat higher performance than a bad algorithm.

Since a cache memory has a high speed compatible with that of the associated processor, all the algorithms of a cache memory have to be implemented in hardware. Therefore, the designers of a cache memory have to consider not only how to implement its functions but also how to implenent these functions with practical hardware.

'Traditionally, a cache system is built with a single cache for both data anel instructions. This cache is called as a unified cache, in which case the C.PU's components have only one cache unit to refer to for both instructions and data. The associated processor shares the same cache for data and instructions, which makes more efficient use of a limited resource and lowers the average miss ratios. Also a cache system can be split into two separate caches: one for data, and the other for instructions. One of the major advantages to splitting data and instructions into two separate caches is that conflicts between simultaneous instruction fetches and data reads and writes are climinated [9].

\subsection{The Line Size Choice}

The performance of most computers depends strongly on the puality of the carlie. design and the way in which it is implemented. Therefore, cache design is a very significant part of computer system design. In order to design a high-performance cache memory, there are several choices to be made and paraneters to be set. 
Designers have to make decisions alout the algorithms (felcl, placement, etc.), about the best sizcs (cache size, line size, etc.), and about the ways of address mapping and maintaining consistency arnong several cachiss in a multiprocessor. Designers also have to make tradeolfs in setting these parameters; e.g. cache size, line size, the set-associativity, and so on. Each of these parameters affects cache performance; choosing different parameters produces different cache performance.

The cache line size is a very important parameter that strongly affects the cache performance, especially the cache miss ratio [11]. Many surveys of cache memory and/or memory hierarchy performance have been made for high performance systems. In these surveys, the cache line size choice, with the overall cache size, has becn shown to strongly alfect the cache niss ratio. Smith suggested in [9] the line size giving the minimum miss ratio for a given cache memory capacity. He also indicatcd that the minimum number of elements per set in order to obtain an acceptable miss ratio is 4 to 8 . Beyond 8 , the miss ratio is likely to decrease very little. After a great number of simulations, Sunith [11] presented practical values for the miss ratio as a function of cacle size and line size which are listed in Table 1. The Design Target Miss Ratios (DTMR) shown in Table 1 are proposed for unificd caches, instruction caches, and data caches, respectively. 'The D'MTR provide designers with a reference to implement a variety of now systens. It can be used to estimate the porformnnce impact of certain design choices. The models of anche nemories for the I'T'Mle assume demand fetch, copy-back caches with a LRU replacement algorithun. Thoy also are full-associative for address mapping, 


\begin{tabular}{|c|c|c|c|c|c|c|}
\hline \multirow{2}{*}{$\begin{array}{c}\text { Cache Type: } \\
\text { Unified }\end{array}$} & \multicolumn{6}{|c|}{ Miss Ratio } \\
\hline & \multicolumn{6}{|c|}{ Line Size: } \\
\hline Size & 4 & 8 & 16 & 32 & 64 & 128 \\
\hline 32 & 0.717 & 0.556 & 0.5 & 0.75 & & \\
\hline 64 & 0.686 & 0.488 & 0.4 & 0.48 & 0.72 & \\
\hline 128 & 0.674 & 0.467 & 0.35 & 0.33 & 0.428 & 0.686 \\
\hline 256 & 0.643 & 0.42 & 0.3 & 0.258 & 0.276 & 0.386 \\
\hline 512 & 0.596 & 0.39 & 0.27 & 0.216 & 0.197 & 0.257 \\
\hline 1024 & 0.473 & 0.309 & 0.21 & 0.162 & 0.137 & 0.151 \\
\hline 2048 & 0.405 & 0.258 & 0.17 & 0.124 & 0.098 & 0.093 \\
\hline 4096 & 0.329 & 0.193 & 0.12 & 0.082 & 0.059 & 0.05 \\
\hline 8192 & 0.232 & 0.135 & 0.08 & 0.05 & 0.033 & 0.025 \\
\hline 16384 & 0.182 & 0.103 & 0.06 & 0.036 & 0.23 & 0.016 \\
\hline 32768 & 0.124 & 0.07 & 0.04 & 0.024 & 0.014 & 0.009 \\
\hline \multicolumn{7}{|l|}{$\begin{array}{l}\text { Cache Type: } \\
\text { Instructions }\end{array}$} \\
\hline 32 & 0.725 & 0.478 & 0.33 & 0.247 & & \\
\hline 64 & 0.674 & 0.438 & 0.3 & 0.222 & 0.191 & \\
\hline 128 & 0.615 & 0.397 & 0.27 & 0.197 & 0.164 & 0.157 \\
\hline 256 & 0.592 & 0.373 & 0.25 & 0.177 & 0.138 & 0.129 \\
\hline 512 & 0.562 & 0.348 & 0.23 & 0.159 & 0.119 & 0.108 \\
\hline 1024 & 0.504 & 0.308 & 0.20 & 0.134 & 0.098 & 5.084 \\
\hline 2048 & 0.391 & 0.234 & 0.15 & 0.098 & 0.068 & $0.05 i$ \\
\hline 4096 & 0.271 & 0.161 & 0.1 & 0.063 & 0.043 & 0.032 \\
\hline 8192 & 0.172 & 0.1 & 0.06 & 0.037 & 0.023 & 0.016 \\
\hline 16384 & 0.148 & 0.085 & 0.05 & 0.029 & 0.018 & 0.012 \\
\hline 32768 & 0.091 & 0.052 & 0.03 & 0.017 & 0.01 & 0.007 \\
\hline \multicolumn{7}{|l|}{$\begin{array}{c}\text { Cache Type: } \\
\text { Data }\end{array}$} \\
\hline 32 & 0.731 & 0.611 & 0.55 & 0.715 & & \\
\hline 64 & 0.66 & 0.515 & 0.45 & 0.495 & 0.693 & \\
\hline 128 & 0.561 & 0.412 & 0.35 & 0.351 & 0.467 & 0.677 \\
\hline 256 & 0.47 & 0.337 & 0.28 & 0.272 & 0.326 & 0.456 \\
\hline 512 & 0.345 & 0.246 & 0.2 & 0.191 & 0.215 & 0.282 \\
\hline 1024 & 0.283 & 0.211 & 0.16 & 0.138 & 0.14 & 0.161 \\
\hline 2048 & 0.256 & 0.169 & 0.12 & 0.094 & 0.083 & 0.089 \\
\hline 4096 & 0.247 & 0.153 & 0.1 & 0.07 & 0.054 & 0.048 \\
\hline 8192 & 0.214 & 0.129 & 0.08 & 0.053 & 0.039 & 0.032 \\
\hline 16384 & 0.161 & 0.097 & 0.06 & 0.039 & 0.26 & 0.019 \\
\hline 32768 & 0.108 & 0.065 & 0.04 & 0.025 & 0.017 & 0.012 \\
\hline
\end{tabular}

Table 1: The Design Target Miss Ratios 


\begin{tabular}{|l|c|c|}
\hline \multicolumn{3}{|c|}{ CACHE TYPE ADJUSTMENTS } \\
\hline Cache Type & $\begin{array}{l}\text { Ratio of Miss Rate } \\
\text { to Dircet Mapping }\end{array}$ & $\begin{array}{l}\text { Ratio of Miss Rate } \\
\text { to Full Associative }\end{array}$ \\
\hline direct-mapped & 1.00 & 1.515 \\
\hline two-way set-associative & $\mathbf{0 . 7 8}$ & 1.182 \\
\hline four-way set-associalive & $\mathbf{0 . 7 0}$ & 1.061 \\
\hline cight-way set-associative & $\mathbf{0 . 6 7}$ & 1.015 \\
\hline full associative & $\mathbf{0 . 6 6}$ & 1.000 \\
\hline
\end{tabular}

Table 2: The Relevant Cache-mapping-type Ratio

except for those with 4 and 8 byte line sizes, which are 4-way set-nssociative. The cache uniss ratio is also related to the mapping methods used. 'T'here are three mapping methods: direct-mapped, S-way set-associative, and fully associative. These arc described in the next chapter. Values in Table 2 express the relative ratios of miss rates based on both the direct-mapped and full associative mapping methods. These cache type adjustments originally are from [30]. They are based on the direct-mapped method, and are expanded to be used for those based on the full-associative method. Since the miss ratios shown in Table 1 are bascd on the full nssociative model, in order to estimate the actual miss ratio of other systents, the final act,ual miss ratio can be obtained by multiplying the given miss ratio found in Table $\mathrm{t}$ by the corresponding relevant cache-mapping-type ratio from colunn Uhrce labeled Ratio of Miss Rate to Full Associative of Table 2. 


\subsection{A Survey of Cache Design}

Since IBM Corporation introduced the first commercial cache menory in its Sirst rm $360 / 85$ to bridge the speed gap between the processor and main momory, variuns cache memories have been omployed in different types of computers to ithinese higher performance. A number of approaches have been used for doveloping high performance cache memories. Although the operation of at typical cache memeny. seems relatively simple in concept, implementation of a realistic caclar momory is quite complex, involving many factors which influcnce cache performantes. These factors involve internal factors such as cache capacity, line size, adderss mappoing strategy, fetch algorithm, placement algorithm, replacement algoritlım, as will at the swapping algorithm, and external factors or system fictors; processor organization, hierarchical memory organization, as well as the interconnection uetwork. such as the system bus. For supcrcomputers, synchronization is in mor serions problem since at least two or more processors are cmbedded in the system. "I lwere fore, attempting to ovaluate cache performance exactly in a realistic compum system is quite difficult. We can, however, use approximate moxlels for mallation of cache behavior and performance.

Cache performance can be described with reference to two ispects [?]: callu. miss rate and access time. The first aspect is cache access time -- the time reopirel for the processor to get information from or store information into the carche, C:a lie. access time depends not only on the design itself but also on the ferlunclogy uneet in cache design. Therefore, the effect of design changes on arcess time is dillienlu 
to predict without specifying the circuit technology used. The second aspect is the miss ratio of the cache memory - the fraction of all memory references attempting to accoss data which are not resident in the cache memory. In general, every cache niss makes the processor wait until the desired data can be received. The niss ratio is related not only to how the cache design affects the number of misses, but also to how the machine design, including harrware and soltware, alfects the number of cache references (main memory references). For example, the cache miss ratio depends on the progran locality implied by software and the amount of information (one word, two words etc.) obtained by the processor at a cache relerence.

Many computer systems (alhost all modern supercomputer and large compuler systems) have cache memories of various designs to bridge the speed gap between processor and main memory in order to improve system performance. T'his section presents a survey of cache memories and their performance in several typical cache. based computer systems.

A high-speed caclie memory was employed in the IBM System 370 Model 168. The cache was available in a size of either $8 \mathrm{k}$ or $10 \mathrm{k}$ bytes. The $8 \mathrm{k}$-byte cache menory had a cycle time of 80 us (the same as the machine cycle time) for accessing 4-byte data. It was organized into 64 sets as a 4-way set-associative cache. The write-through schene was used for updating the main memory. The average miss ratio was about 7 percent $[27]$, and the uniss ratio prediction, according to the DTMR, is 5.3 percent. 
The IBM 3033 has a $64 k$-byte cache memory for both instructions and data with 57 ns cycle time. This large, high-specd cache memory is one of the main reasons for the high performance enhancement of the 3033. This cache is organized into 64 sets as a 16-way associative cache. The line size of the IBM 3033 is 64 bytes. Also the write-through policy is employed in the IBM 3033. In this systen, the main memory is divided into 8 modules so that main memory can transfer a line by interleaving [5].

The VAX-11/780 is a 32-bit high-performance minicomputer first introduced by DEC in 1978. Its caclie has $8 \mathrm{k}$ byte capacity organized into 512 sets, two lines per set, and 8 bytes ( 4 bytes per word) in each line [5]. For the cache menory of the VAX-11/780, a distinction is made between a read and a write miss. If there is a read miss, the required line has to be retrieved from the main memory and writlen into the data cache. If two lines in the given set are full, some sort of line replacement strategy has to be employed to determine which line is swapped with the new required line. The $\mathrm{V} \wedge \mathrm{X}-11 / 780$ cache memory uses a rundom replncemrnt stralegy as its policy for updating the line. If there is a uniss cansed by a write operation, only the referenced location of the main memory is updated. This data cache uses a buffered write-through policy. The miss ratio of VAX-11/780 was measured to be about 13.05 percent [34], and it is also estimated to be 13.5 percent. by the DTMR.

Today cache menories have been integrated with their corresponding microprocessors on a single chip, giving so-called on-chip cache menories. The Z80000 
microprocessor produced by Zilog in 1985 includes a 256 byte on-chip cache memory which is organized into 16 lincs, 16 bytes each, as a fully associative cache. The maximum clock frequency for the Z80000 is $25 \mathrm{NHIZ}$, and when the Z80000 fetches from its caclie, only one system clock cycle is required [28]. The least recently used line (LRU) replacement algoritlum is used to choose the line to be replaced by the new one from the main memory in the case of line-miss occurrence. The writcthrough algorithm is used in this cachc for its writing strategy. When there is a miss caused by a write operation, only the main memory is updated. This cache has a niss ratio of 25 per cent for a no burst transfer mode and 12 percent for a. burst irausfer mode [29]. It is predicted to have, as a unified-cache, a miss ratio of 30 per cent using the DTMR.

A cache memory has also been applied to the Balance mulliprocessor system introduced by Scquent Computer Systems Inc. in 1988 [37]. This multiprocessor system can pool up to thirty 32 -bit processors with a shared main memory. A subsystcm in this system is composed of an NS32032 microprocessor, an NS32081 foating-point unit, and an NS32082 paged virlual memory management unit, produced by National Semiconductor. In addition, each subsystem has an $8 \mathrm{k}$-bytc two-way set-associative cache memory to achieve a high performance while minimizing bus traffic. In this cache, with a 50 ns cycle time, there are 512 sets, two lines cach, and 8 bytes per line. The write-through policy is employed to kcep all the copies in the system consistent. Whenever there is write request from one processor in the system, this request with the corresponding addross is sent to update 
stale data in the shared memory while it is broadcast to all the caches to see if there are any copies of the data to be updated. If so, the corresponding caclie controller invalidates the affected line. The miss ratio of a single-thread cache memory is 15 per cent [37], while the predicted miss ratio from the DTMR is 15.9 per cent.

Since the caclie miss ratio is very dependent on the programs that execule on the cache-based systems and the models in [11] are ideal (in general, a real catche memory is more complicated, and there are more factors to be considered), we can see that our design target miss ratios are slightly higher than seen in simulations described above, and close to those from measured ıesults, such as for the VAX$11 / 780$, which lends some credibility to the use of the D'TMR as a rensonable estimator of cache performance, as noted in [11]. 'T'hus, the set of design target miss ratios is very useful for design and implementation of a possibly new cache or architecture. Also we can see that the line sizes of the systcms discussed above secm too small. A larger line size provides a lower miss ratio under a fixed cache size. It is clear that caches using set-associativity have lower a miss ratio than those using thr direct-mapped method. Another problem is that the above systems which use setassociativity have a small sel size, which affects the cache miss ratios. In adelition, for implementations of existing cache memories, atmost all caches are implemented in either multi-chip or on-chip configurations. In the case of chip sets, several chips, including one cache controller and several high-speed static RAM chijs, are used to build a cache memory. This kind of cache memory is designed for special processors and has a fixed caclie size. 'They do not have unch flexibility; for example, the: 
cache size can not be changed after the cache controller is designed, and they have longer delay time between the cache controller and RAM chips. An on-chip cache does not have a delay penalty due to interconnertion between the chips of a multichip cache memory, but on-chip caches have the same problem of infexibility as do mulit-chip cache menories. In addition, this kind of eache in general has only a small capacity using today's technology, which leads to a higher miss ratio.

Using VLSI technology, we can make traceoffs to design a novel cache menory which has a larger cache size, larger line size, and higher set-associativity on a single chip with little delay penalty by eliminating the wire-connection delay between the cache controller and the cache data memory. Multiple uniform cache chips can be used to build cache systems of various sizes, associated with one processor. This cache system can be used as a traditional unificd cache for both instructions and data, or as separate instructions or data cache. 


\section{IMPLEMENTATION OF THE CACHE ALGORITHMS}

\subsection{Cache Design Parameters}

Typically, a cache memory system can capture well over 90 percent of all references to main memory. Optimization of the cache design parameters is very important to decrease the cost/performance ratio for high-performance cache menorics. Optimizing the design of cache memory has four aspects [9]:

1. maximizing the hit ratio

2. minimizing the access time to cache data

3. minimizing delay due to a cache miss

4. minimizing the overhead of updating main memory and maintaining cache coherence

In addition, for cache memories for multiprocessor systems, consideration has to be taken to maximize bus and shared-memory bandwidth and to minimize the bus bandwidth required by cach processor in order to maximize the system performance. There are also trade-offs which depend on the technology of implementation for the cache; for example, between hit ratio and access time.

There are many factors to be considered during cache design which affect system performance. Parameters for cache design are classificd into intrinsic and extrinsic 
parameters [5]. Elfective memory speed and cost are two intrinsic paraneters. Extrinsic parameters, such as hit ratios, control algorithms, etc., are selected based on the results of experimental data and simulation, and are variables which must be considered for the system design,

Of all the considerations which are related to cache memory, the following are mainly considersd during design since cache performance is sensitive to choiets concerning these aspects:

1. Fetch policies

2. Mapping policies

3. Replacement policies

4. Swapping policies

5. 11 it ratio and access tine

6. Cache menory capacily

7. Line size

8. Cache dala path width

9. Main menory organization

Fetch algoritlums are used to determine when the system brings information into the cache memory. In general, the major fetch algorithms are demand-fetch and prefetch. Under the demand fetch algorithm, a line is fetched only if it is 
needed. The prefetch algorithm, on other hand, gets information before it is noeded. Therefore, the prefetch algorithm is based on some kind of prediction about which line will be used next. It must be designed carefully if the machine performaner is to be improved rather than degraded [9]. Implementation of a prefeteh algorithm is usually more conplicated than demand fetch.

Mapping policies are used to translate the logical address space to real addiress space. Efficient address translation schemes should accomplish address translation in such a way as to minimize the apparent access time. Information gencrally is obtained from the cache associatively; larger associative menory is more expensive and slower. Hence, there must be some trade-off of associativity during cache design, in terms of the design and technologies that are employed. A mapping such that any of the lincs in main menory can be mapped into any line slots in caclie memory is called a full associative mapping. That is, a line of main memory may be mapped into any location of the cache memory. Typically, length of a line in cache memory is as the same as that of main memory. If the cache menory is full and there is a miss, the requested line can be transferred into any line slot of cache memory from main memory, in a manner depending on the replacement policy employed. Thus this mapping provides the minimum probability for line slot contention problems and the largest hit ratio for a given problem. Ilowever, having one comparator per address tag makes it very dificult and costly to implement, especially in a large cache memory.

A direct-mapped cache has only one comparator which is connected to all the 
address tags in cache memory. Each time only one address tag can be selected to compare with the address from the processor. This mapping is a many-to-one mapping. That is, any given line in main memory can reside logically only in one specified line slot in cache memory. A direct-mapped cache memory mandates a fixed replacement policy; if there is a line miss, both the cache tag and the corresponding line are replaced with the requested main menory address and its line. This mapping has the highest probability of cache memory slot contention since there is a fixed replacement schene. Furthermore, it gencrally has a rolatively low hit ratio. Unlike the full-associative may,ping, it is quite simple and easy to implement.

A third mapping method is an S-way set-associative mapping, which is a hybricl of the dircet-mapped and full-associative methods. An S-way set-associative cache has multiple sets which can be selected by direct-mapping, and there are $\mathrm{S}$ lines slots in cach set which can be simultancously compared with the address from the processor. In this mapping systern, there are $\mathrm{S}$ comparators, a comparator for each "way". Set-associative mapping has a reasonable implementation complexity and hit, matio. Increasing the cache size of a sct-associative cache gives a greater hit ratio than increasing the depth of a direct-mapped system. On other hand, increasing the number of sets, or ways, of a set-associative cache memory also gives a greater hit ratio. llence many high-performance cache memories, especially large scale caclics, adopt the set-associative mapping mechanism as a compromise between complexity and performance. More details about S-way set-associative mapping 
are given in the next chapter.

An optimal replacement policy would predict the line which will be used in cache memory (or a given set) furthest in the future and which consequently slonld be discarded when the cache memory (or a given set in cache menory) is full aurl a cache miss occurs. This policy would keep data in the cache optimized for the highest hit ratio, and the maximum system throughupt. However, this optimal replacement policy can not be implemented since it requires a prediction of the future behavior of the running programs. Therefore, some approximation has to be made. There are three types of practical replacement algorithus commonly used for cache momory systems; first-in first-out (FIFO), random, and least recently used (LRU) line replacenent, to approximate this function. The FIFO algoritlum is based on the principle that the first line to be referred is predicted to be the line not to be used in cache memory (or in a given set) furthest in the future, and that this line is replaced by the new one from main menory. This algorithm does not really reflect the program locality very well, since the first line may b: used frequently, but it is easy to implement. The random scheme is based on at random number from a random number generator to create the line number of a line which is replaced by a new one whenever there is a replacement necd. $A$ cache memory employing this algorithm typically has a low hit ratio since this algorithm is not able to reflect the program locality. The least recently used line replacement algorithn, which looks backward (past), is usually able to reflect the program locality well since it is based on historical line usage. 'That is, the least 
used line in the recent past is replaced by the requested line from main memory. Since this algorithm requires more stored information about the past, it is more difficult to implement in hardware, especially in a large scale cache memory. A variation, an approximation of the LRU algorithm, can be used to simplify the hardware implementation. This variation is based on the fact that if a line has not been referenced over a certain time period, it is less likely to be needed next than lincs in caclie memory (or in a given set) that have been referenced in that period. More cletails of the least recently used line replacement algorithm are described in the next section. No one best algorithm exists from the practical replacenent algorithms [3]. Some algorithm, compared with the other algorithuns, is better for particular classes of problens and poorer for other classes. However, in general, the LRU algorithm is clearly the best choice for most applications, since it is based on historical line usage (the rocent past appears to be a good cstimate of the near future), it works well, and it increases the hit ratio when the number of lines is increased.

Swapping algorithms are designed for transferring a new line from the main memory to the caclie wlien the requested information is not in the cache. Typically, there are two kinds of swapping algorithms: write-through and copy-back. In the writc-through scheme, a processor write to cache memory is immediately writtcn through to main momory as well. Therefore, the information in both cache tnenory and main memory is always consistent. Purthermore in a multiprocessor environment, it can handle multiple-cache colıerence in an easy way. Unlike the 
write-through scheme, the copy-back scheme (without line miss occurrences) only updates the copies of requested data in cache memory withoul disturbing main memory. Whenever there is a line-miss, cache memory copies back the line to be overwritten to main memory before transferring the requested line to caclie menory. It can reduce traffic between cache memory and main menory. Ilowever, it requires more complicated logic; and there is a colerence problem betwesen cache memory and main memory, and potentially between multi-caches in a multiprocessor system. In contrast, the wrile-through method has higher traffic between cache memory and main memory since write operations vary from 10 percent to 30 percent out of total references, depending on proccssor architccture and the particular set of applications. The average percentage of write operations in [9] is 16.

The hit ratio for a cache memory is defined as the probability, or the fraction of times, that a memory request is found in cache memory. If we define the probability of all the references to memory as 1 , the miss ratio of caclie memory is (1-hit ratio). The hit ratio for a cache memory is one of the most inportant, fuctors for the performance evaluation of cache memory. Olher important factors affecting the cache performance are the access time for the caclic memory, including time to search the directory, and the cache memory cycle time, which is defined as the time the processor accesses information in cache menory. The access time of a cache memory is affected not only by the architecture, or design (including all the algorithms and parameters selected in cache design and implementation of the 
algorithus in hardware), but also by the technology adopted (bipolar, CMOS, ctc).

The caclic capacity is usually dictated by many factors having to do with the system cost and performance. In general, a large cache capacity can produce a higher hit ratio, and in turn a better performance. However, there are some linitations on cache size beyond which cacle inemory has either a high cost or performance decreascs due to the long access time.

The line size of cache menory is one of the most important parancters which sensitively affect cache performance. There are a number of trade-olfs for a reasonable line size in terms of architecture and technology. Using VLSI technology, a larger line size is preferred because it achieves a lower miss ratio without much cxtra cost. But if it is too large, it increases line transfer time and, in turn, decreases system speed even if the hit ratio is increased. It also depends on the clata path width between cache and main memory.

The cache data path width must be considered during the design process since it clirectly deternines the time required when a line is transferret from main memory to cache memory. From the point of view of performance, the cache data path should be as wide as possible. It is clear, however, that cache data path is expensive. Doubling the path width nocans doubling the number of lines in and out of the cache and all the associahed circuitry. The path width is critically important to caches implenented using VLSI technology because of the limited number of 1/O pins on a chip. Hence, a trade-ofl of the cache data path width lias to be made during the cache tlesign to achicve a reasonable cost/performance. 
Although the use of cache menory in computer systems can gratly reduce direct references to main memory, memory traffic is still a very significant performance factor, especially in a multiprocessor system. Memory traffic con1sists of two components: fetch traffic and write-through or copy-back traffic. The fetch traflic arises from the transfer of data from the main menory to the cache while the write-through or copy-back traflic is from the cache to the main menory. The fetch traffic can be obtaincd by multiplying the miss ratio by the line size to get traffic in bytes/reference. 'The write-through traflic can be calculatexl by multiply. ing a write ratio (the ratio of writes to total references) by the number w bytes per write operation. Similarly, the copy-back traffic can be determined by multiplying the miss ratio by the line size, since a line niss causes writing of an existing cacle line in the cache into the main menory before transferring the requested missing line to the cache. For evaluation of a cache-based multiprocessor system with a single bus, a bus utilization can be used to estimate the memory traffic. 'The bus utilization is defined as the ratio of time spent doing useful work to the total run time of the bus.

Since decreasing memory tralfic or transfer time during a line miss can incrcase the system performance, optimization of the organization of both the main memory and interconnection network is a key factor for high system performance and low cost. For the interconnection network, a wide data path can reduce the transfer time, but the cost is much higher. On the other hand, if the main memory is made: up of several modules which can operate independently, traflic can be reduced 
because more than one module can be busy writing at one time. Furthermore, if modules can transfer different words in a line by interleaving, trans[erring a line from main memory to cache onl- takcs one main memory cycle. Thus, the main memory bandwidth can be increascd while the transfer time is greally decreased.

\subsection{The Structure of the Cache Memory}

During the design of the caclic memory described here, algorithms and parameters used have been selected carefully, and a number of trade-offs between them have been made in order to achieve high performance. The cache memory system described here is implemented as a single chip. Furthermore, this implementation allows a cache of variable capacity (larger than the capacity of a single caclie chip)) by using several of the cache menory chips. The single-chip cache memory described here has a capacity of $8 \mathrm{~K}$ bytes because of silicon area limitations for the 3 micron CMOS technology. The word size for this cache memory is 32 bits since this cache is designed for a 32-bit computer system. A word is not necessarily the suallest unit that the processor can access. The processor can directly access 1, 2,3 , or 4 bytes from the cache. Therefore, it provides more flexibility to computer systems in which the cache is used. It also allows for the possibility that this cache can be used in 16-bit conputer systems, provided control signals for the cache can connect with that of the processor with reasonable additional logic. Two clock phases, $C K 1$ and $C K 2$, are employed to pipeline this system. Each of the clock

phases has a minimum cycle period of 36 nanoseconds (derived from simulation) 


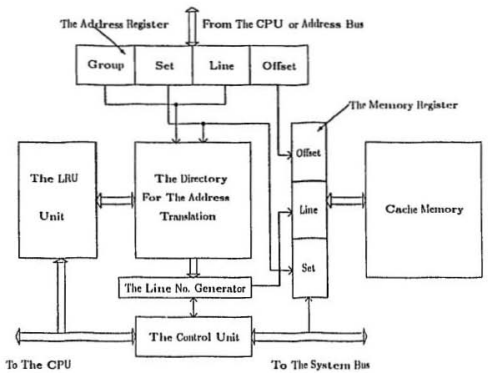

Figure 3: The Basic Cache Menory Structure

in which the associated processor can read an instruction or data from the cache.

Fig. 3 depicls the structure of this cache memory. Il is composed of four basic components as follows: the Address Translation Function or Directory, the Line Replacement Unit (LRU), the Cache Memory and the Control Unit.

During $C K 1$, the address fron the processor is latehed in the address register of the cache, and then it is sent to the directory to see if the lins containing the requested data is in the cache. If so, the line number generated by the line number generator, the set number from the address register, and the word offset in the line are all combined to form a word address for the cache memory and latcherl into the cache memory register. In addition, the proper byte(s) can be accesscel 
by the processor using both the two least significant bits of the address from the address register and two function bits from the processor, which will be described later. Mcanwhile, the LRU unit is updated to indicate that the line referenced is the inost recently used onc in the given set. During $C K 2$, a read/wrile operation is done. If the requested data is not in the cache, a line miss occurs. The LRU unit is asked to send the least recently used line number in the spocified set to the directory, and the directory uses this number to locate its corresponding line slot in the specified set of the directory. Then the contents of this slot are replaced with the group number and the line number in the address register. After replacenent, the line number generator gives the line number corresponding to this cell to the memory register to transfer the repitcsted line from the main memory to the cache. To obtain the line of information from main memory, a line miss signal is sent to main memory. After the caclic receives a "bus use" grant from the system bus controller, the processor is forced to be idle during transfer. There are 8 words (32 bytes) to be transferred from the main memory to the cache memory during a line miss, which would normally take a long time and in turn decreases system performance. In order to reduce the line transfer time, the majn memory may be partitioned into several modules, or "interleaved" (in this case, the memory should be partitioned into 8 modules). Whenever there is a line miss, the 8 words of the requested line can then be sent to the caclie memory almost simultaneously, with each word coning from a separate module of the main memory. Thus, the transfer line can greatly be decrased. 'The main memory organization will be discussed 
later in chapter 5 .

\subsection{The Address Space Mapping}

Since the eache, as the fastest pari of the memory hierarchy, is much smaller than the main menory, there has to be a mapping function between the cache address space and that of the nain memory. As discussed previously, the direct-mapped method is the simplest to implement, but it has the highest miss ratio of three mapping methods. 'Therefore, it is not used in this appliation. Nso the fully. associative metliod requires one comparator per line slot it the diretory. This is costly to implement in a large scale cache menory. Also, it may introducx an extra tag-scarch delay and make the search logic complicated. The sct-associative method, a hybrid of the direct-mapped method and the fully-associative method, is used in this mapping mechanism. It involves organizing the cache menory into $S$ sels of $N$ lines per set. When $N$ becomes one, the cache is a fully-associative cache in which there are $S$ sets in total, each consisting of a single line. If $S$ becomes one, the organization of the cache is the direct-mapped cache memory. Since an S-waty set-associative cache allows any one of $S$ lines in a referenced set to be replaced on a line miss, this flexibility usually introduces a lower miss ratio without the complexity of a fully-associative cache. Therefore, it is a comprounise between complexity and performance. 


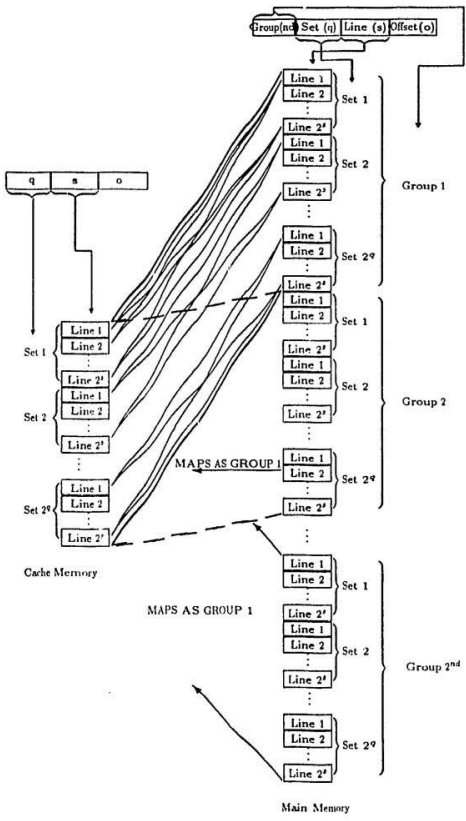

Figure 4: The Set-associative Mapping 


\subsection{The Set-associative Mapping}

The principle of set-associative mapping is shown in Fig. 4. The cache menory is divided into $2^{y}$ sets wilh $2^{*}$ line slots in each, and the sizes of the sets and lines in the cache menory are the same as those in the main memory. Furthurmore the main memory is partitioned into several groups, and the size of ench group is equal to the size of the cache menory. Hence, each group contains $2^{q}$ sets. Each set slot in the cache menory must be shared by several scts of the main mctnory. For example, in Fig. 4, the first set in the cache memory is assigned to hold the sets $1,1+2^{q}, 1+2 \times 2^{q}, \cdots$ of the main memory and the second sct is assigned to hold the sets $2,2+2^{q}, 2+2 \times 2^{q}, \cdots$ and so forth. Lines within a set of the main memory are associatively mapped into any of the $2^{s}$ line slots in the corresponding set of the cache memory. That is, any set in the main memory cau only be directly mapped to a specific set of the cache menory and lines in a set are associatively mapped into any of the $2^{s}$ line slots in the corresponding set. Sets fron different groups can be intermixed within the cache memory; therefore not all the sets of a given group need to be simultaneously resident in the cache menory; sintilarly lines in the sets from different groups, which are mapped into the same set of the cache memory, can also be intermixed within that set of the cache memory. E.g. line 1 of set 1 of group 1 can be assigned to line slot 2 of set 1 in the cacle menory and line 2 of seb 1 of group 2 can also reside in line slot. 1 of set 1 at the: sane time.

In the Fig. 4, we can see that a memory address is divided into four part.s: ud represents the group number, $\eta$ is the set number, $s$ is the line number and o is the: 
word oflset within a line.

\subsection{Implementation of the Directory}

In gencrul, iucreasing the degrec of associativity of a cache memory can decrease the miss ratio of a cache. In order to obtain high performance, an 8-way set-associative mapping is employed in this directory to achieve a hight hit rate without the extra delay penalty while senrching the dircctory. 'Tlis directory can map the main memory address space ( 32 bit) to the cache menory address space (13 bit) in a maximum of 16 nanoscconds, including the delay of the LRU unit, detcrmined by simulation. Fig, 5 shows the organization of the address mapping directory with set associative mapping. When the processor requests a read/write operation, the logical address is mapped into the cache menory address by searching the directory. 'This directory has a tag array of 32 sets with 8 line slots in each set. Each set is represented by a row of the tag array and the 8 line slots in a given set arc indicated by columus of the tag array. Therefore, this directory is an 8-way set-associative directory in which cach column represents one way.

There is a MATCII signal for each column of slots. If the signal is set, the requested line slot is in this column of slots. Among the 8 MATCII signals, Cach of which connects to a column of the tag array, there is only one MATCH signal valid at any time since only one slot may be selected. All the MATCII lines are connected to a line number generator. The line number generator can translate the ith column number, at which the corresponding $M A T C H$ signal is 


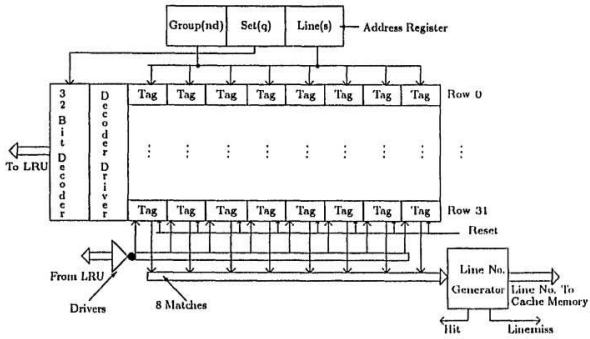

Figure 5: The Directory 
valid, into a binary number $i$ forming the requested line number for the cache memory. That is, the slot at the position in the ith row and the $j t h$ column can produce the line number $j$ of the $j$ th line of the ith set of the cache memory via the line number generator. In each line slot, the group number is concatenated with the line number $(n d+s)$ of a logical address, which indicates that the sperified line of the main memory resides in the cache line indicated by the line slot. Whenever a reçucsted set is selected through the 32-bit directory decoder after an address is lateled into the address register, the contents of the 8 line slots in the selected set are simultaneously compared with $n d+s$ of the address register. If the contents of any one of the 8 line slots are the same as $n d+s$, then the requested data are in the line nf the selected set, and the corresponding MATCH signal slould become valid to make the litie number generator produce the requested line number for the cadie memory. A IIIT llag is also generaled by the line number generator to indicale that the requested data are in the cache. The line number is combined with the set number and the word offset in the line from the address register to form the required word address of the cache memory. Meanwhile, the IIIT flag informs the LRU unit to update the records in the selected set. Otherwise, a MISS flag is set to indicate that the requested data are not in the cache, at which time there are three tasks to be done:

- Invoke the LRU replacennent unit to find the least recenlly used line in the selected set of the caclie. This line will be replaced by a new one when the requested data are available from the main memory. 
- Inform the CPU that it must be idle during the line replacement.

- Request the main memory to transfer the required line to the cache.

Fig. 6 shows a simulation for the tag array of the directory for the case where a line niss occurs during a read/write operation, and the address residing in slot $i$ of row $j$ is replaced with that in the address register. Signals $B_{0}$ to $B_{21}$ represent the $n d+s$ from the address register. $S E L_{j}$ is a signal from the decoder to select row $j$ of the directory. $\Lambda W O R D_{i}$ is a sigual from the LRU unit to update the line slot in row $j$ and column $i$ of the tag array during a line miss; and a $I I T_{\mathrm{i}}$ is the ith MATCII line of the directory in Fig. 5, indicating whether or not the corresponding colunt is malched. After the tag array is reset by the RES, a miss (a low $H I T_{i}$ ) is produced since the $j$ th row of tags selected by $S E L_{j}$ is empty. The signal $W O R D_{i}$ from the LRU unit updates the slot in row $j$ and column $i$ of the directory. After a delay of 6 nanoseconds, the $H I T$, signal becomes high to indicate that the updating has been finished. When the signals on $B_{0}-B_{21}$ are clanged to a new address and the new address is not found in the directory, then the $I I I T_{\text {i }}$ signal becomes low after a 3ns delay.

\subsubsection{The Line Slot of the Directory}

As described $b$ fore, the directory is composed of lines slots. Wach of the line slots is uscd to store the group number and line number $(n d+s)$ of a giten main menory address, which indicates that the corresponding line from the main menory is in the cache menory. For each line slot, there is a 22-bit. built-in comparator to be 


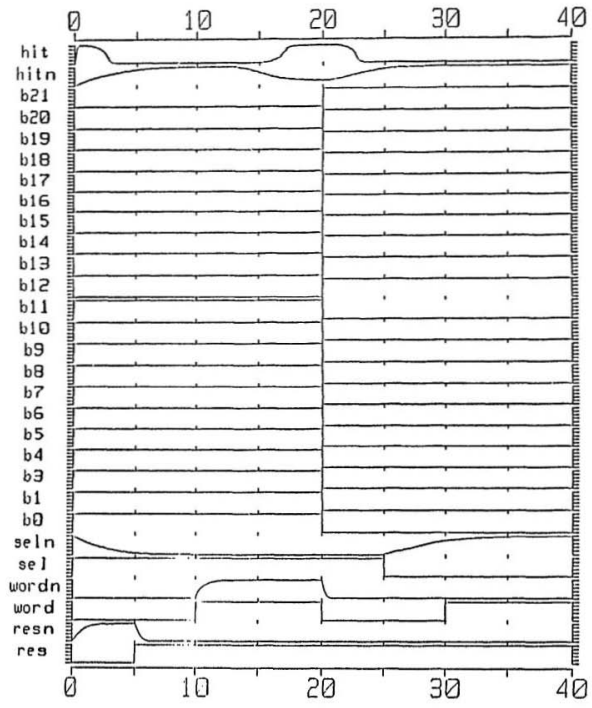

Pigure 6: Simulation of the Tang Array 


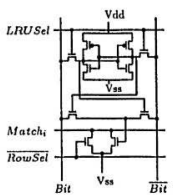

(a) A Tag Bit of The Directory

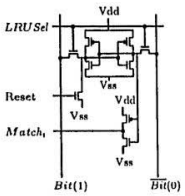

(b) A Valichtion Bit

Pigure 7: The Directory Tag
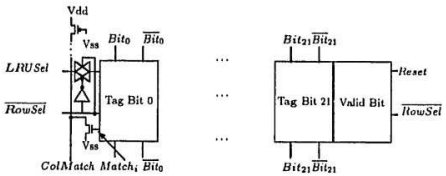

Figure 8: The Thg Bit and Valid Bil of the Directory 
used for parallel comparison of its contents with the $n d+s$ bits of a main memory address. The organization of a line slot (or line tag) is shown in Fig. 7. There are 22 bits for $n d+s$ (19 bits for $n d$ and 3 bits for $s$ ) and one valid bit. If the valid bit is reset, the content of this slot cannot be compared with adts and the directory simply sets a line miss. Otherwise, if $\overline{R O W S E L}$ from the inverted driver connccted to the 32-bit decoder is at logical 0 , the contents of the line slot are compared with $n d+s$ to sec if the requested data reside in the cache. If all the bits of a line slot mateh the $n d+s$ on the lines $\left(B I T_{0}, \overline{B I T_{0}}\right)-\left(B I T_{21}, \overline{B I T_{21}}\right)$, the $M A T C H_{i}$ signal for the line slot becones high to turn on the $\mathrm{N}$ type derice so that the $\overline{C O L A T T C M}$ signal is pulled low. If any bit of the line slot does not mateh the corresponding bit of $n d+s$, the $M A^{\prime} T^{\prime} C H_{i}$ signal for the line slot remains low so that $\overline{C O L M A T C W}$ is ligh. If there is a line miss (there is no line slot matching with $n d+s$ in a selected set) and that line slot in Fig. 7 is chosen by the LRU unit as the least recently used line slot, the LRU selection signal LRUSEL becomes high. Since at this tine ROWSEL turns on the pass gate, this line slot is replaced with $n d+s$ so that the missing line will be transferred from the main memory into this cache line.

Fig. 8 (a) shows the circuit for one line slot bit. If the ROWSET line which is connected to one output of the 32 -bit decoder through an inverted driver is at logical 1 (meaning this line slot is not selected by the decoder), the $M A^{\prime} T C H_{i}$ line remains low so that no comparison can be done in any case (it can be considered to mean "not-match"). If $\overline{R O W S E L}$ becones low, this cell becomes a normal 
content addressable memory cell. For a comparison operation, DATA is applied on the $B I T$ line and $\overline{D A T A}$ on the $\overline{B I T}$ line. If the datum matches the value in the bit, then the match transistor $\mathrm{A}$ remains turned of so that $M A T C H_{i}$ is high for this bit. In a line slot, all the $M A T C H_{i}$ lines are cascaded together. If any bit of the line slot does not match the value on the bit input, the match transistor for this bit pulls down the $\mathrm{MATCH}_{\mathrm{i}}$ line of this line slot, previously precharged hy the validation bit, indicating that this slot does not match the $n d+s$. For a replacenent operation, if there is no slot match at all in the selected sel, a line niss has occured. The LRU unit determines which line slot is to be updated with $n d+s$ by asserting the LRUSEL line for the corresponding line slot in the set selected by ROWSEL. As shown in Fig. 8 (a), when the LRUSEL line is asserted, the values on BIT and $\overline{B I T}$ change the state of this bit. After a change of the contents of this line slot, the contents of the line slot always match nd+s. Thus, the COLMATCII becomes low to cause the line number generator to create a miss line numler to transfer the requested line from the main memory to this caclie line. Note that all the COLMATCII lines in one column of the directory are wire-Oled. This mrans that there is only one $M A^{\prime} C I I$ line for each column of the directory. If any one of the COLMATCH signals in a columu switches to a low value, the MATC'II line of this column becomes low to indicate that one of the line slots in this column matches the $n d+s$. 'The exact position of the line slot in this column is locaterl by the 32-bit direclory decoder. For this 8 -way set associative directory, there are $8 M^{\prime} T^{\prime} C^{\prime} l l$ lines connecting to the line number generator which produces in turn 
the corresponding line number for the cadhe memory.

Fig. 8 (b) demonstrates the function of the valid bit. The signal BIT is set to

logical 1 while the signal $\overline{B I T}$ is set to logical 0 . After applying a resel signal to the RESET line, the input of inverter B becomes high to discharge the $M A T C H_{i}$ line so that the line slot can not be compared with the nd+s. If the LRUSEL line is asserted, the input of inverter B becones low so that the $\mathrm{MATCH}_{i}$ line is charged to make this slot active for comparison when $\overline{R O W S E L}$ is low. (This means that the set to which this slot belongs is selected). In this case, the line slot is valid for searching.

\subsubsection{The Address Register}

The address register is used to latch addresses from either the associated processor or the system address bus in the case of a muitiprocessor system. Fig. 9 shows one bit of the address register. During the $A L E$ (Address Latch Enable) period for the processor, (the period when the address becomes stable), the address from the processor, imposed on inputs $\mathrm{AB}$ of the register, is latched in this register. When the cache receives a search interrupt SEARCIIINT from other caches, the clock pulse generator of the control unit produces a pulse $C K 1^{\prime}$. During $C K{ }^{\prime} 1^{\prime}$, the address imposed on the $\mathrm{AB}$ lines from the other cache through the address system bus is latched in this register for an update operation. After being updated, the cache refurns the address before the update operation to the address register during a pulse $C K^{\circ} 2^{\prime}$ generated by the clock pulse gencrutor. At initialization, the $\overline{\text { reset }}$ 


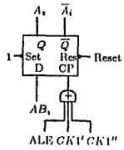

Figure 9: One Bit of the Address Register

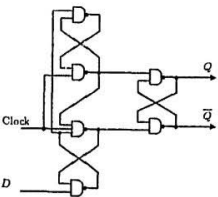

(a) The logieal Circuit

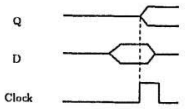

(b) Timing Dingram

Figure 10: The D-type Rising-edge-triggered Flip-flop

signal from the processor resets the register. This register is composed of $32 \mathrm{D}$ type rising edge triggered flip-flops. Fig. 10 (a) shows the logic circuit for this D-typc flip-fop. Note that the delay until an address is valid is merely the signal propagation time in the D flip-flop. Fig. 10 (b) is the timing diagran for the: D-type flip-flop. 


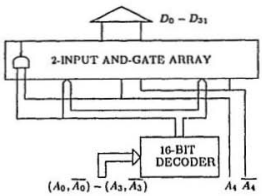

Figure 11: The 32-bit Directory Dccoder

\subsubsection{The 32-bit Decoder for Set Selection}

An address decoder is an essential component for sct sclection in the set-associative directory. A decoder has $n$ inputs and $2^{n}$ outputs. One and only one output will have a value of logical 1 for each combination of input values. In principle, a onc-level decoder could be any number of inputs using $2^{n}$ galcs with $n$ inputs. Unfortunately, in practice, the fan-in limitations and propagation delay require that a large decoder be organized into a multilevel network.

This 32-bit decoder is used to decode 5 bits of the set number from the address register. The inputs of the decoder are the 5 -bit set number and its conplement directly from the address register. The outputs have 32 bits and at any time only one bit has a value of logical 1 . The decoder consists of a 16-bit decoder and 32 2-input NOR-gates as sliown in Fig. 11. A 2-input NOR-gate is preferred for the last stage in the multi-level network to allow fast rise time [20]. Address bits $A_{0}-A_{3}$ and their complenents $\overline{A_{0}}-\overline{A_{3}}$ are imposed on the input lines of the 16-bit 


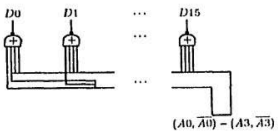

(a)

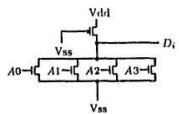

(b)

Figure 12: The 16-bit Decoder

decoder while address bits $A_{4}$ and $\overline{A_{4}}$ are sent to the 16 2-inpul NOli-gates at the second stage of the decoder, respectively. Outputs of the 32 -bit decoiler $D \Lambda_{u}$ lo $D A_{31}$ are sent to the directory to select the corresponding set. The 16 -bit cleccoter is illustrated in Fig. 12 (a) where the 4 -input NOR-gates are cmployed. The 4 . input NOR-gate is implemented with pseudo-nMOS logic as shown in i"ig. 12 (h). There is only a single p-type transistor in the circuit, with the gate connected to $V_{s s}$. Use of pseudo-nMOS technology can decrease the area and deliny time of the decoder since the number of the slow cascaded p-devices is reduced from four to one. The transistor sizes are ratioed carefully to ensure correct logic function and high speed. A sinulation for the 32-bit decoder is shown in Fig. 13, in which the 
time delay is approximately one nanosecond.

\subsubsection{The Line Number Generator}

The circuit for the line number generator is illustrated in Fig. 14. The function of the line number generator is to translate the line numbers of the cache memory from "one-hot code" (or hot code), composed of $8 \mathrm{M} \Lambda \mathrm{TCH}$ lines from the directory, into binary code. In a "hot code" number, there is at most one bit at logical 1; Table 3 is the trutl table for the 8-bit "hot code" and its corresponding binary code. Each bit of the complementary "hot code" corresponds to one of 8 match lines $\overline{\text { ColMatch }}-\overline{\text { ColMatch }}$ from the directory. If all 8 bits of the "hot code" are zcros, then none of the match lines is at logical 0 . In this case, the line number yenerator produces a IINEMISS. If any one of the ColMatch signals is logical 0 after comparison, the generator turns out the cerresponding line number of the cache memory in 3-bit binary code on the ontput lines $I I N E_{0}$ to $L I N E_{2}$; meanwhile, IIIT is asserted. This binary line number is lateled into the line register of the menory register during $A L E^{\prime}$, immediately following. The layout of this circuit is shown in Fig. 15. A simulation for this circuit is shown in Fig. 16 in which the time delay for a valid signal is seen to be about 2 nanoseconds.

From the simulations, the total delay before turning out a valid cache address by the directory for both the miss and hit situations is shown in the Table 4. (The abbreviation LNG stands for the line Number Generator.) If the requested data reside in the cache, the required delay of the directory is about 14ns (1)ecoder + 

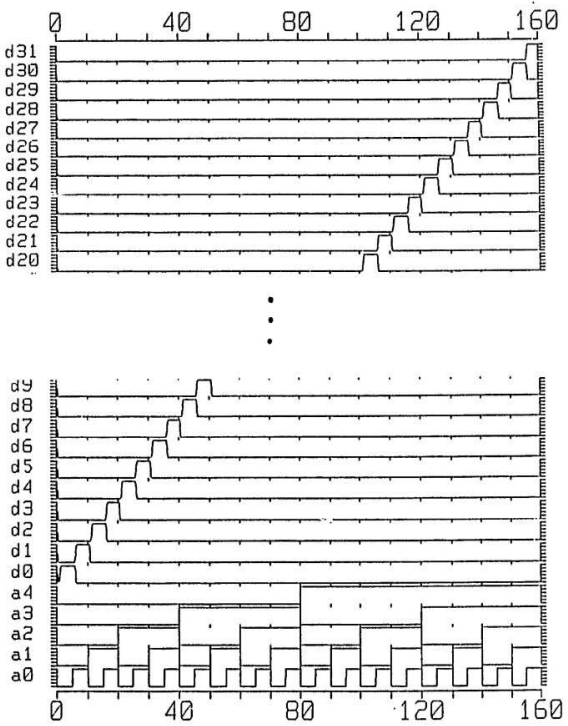

Figure 13: Simulation of the 32-bit Decoder 


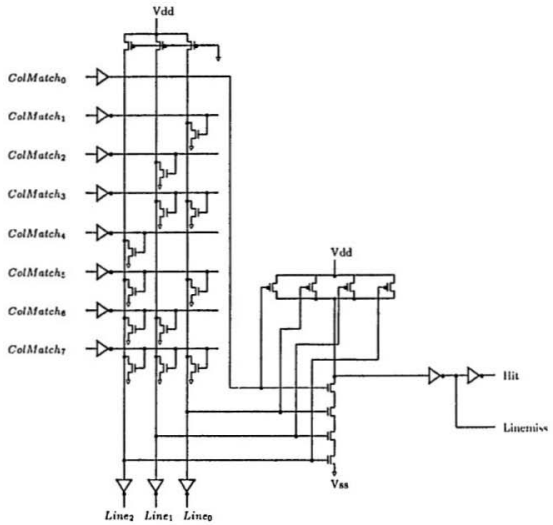

Figure 14: The line Number Generator 


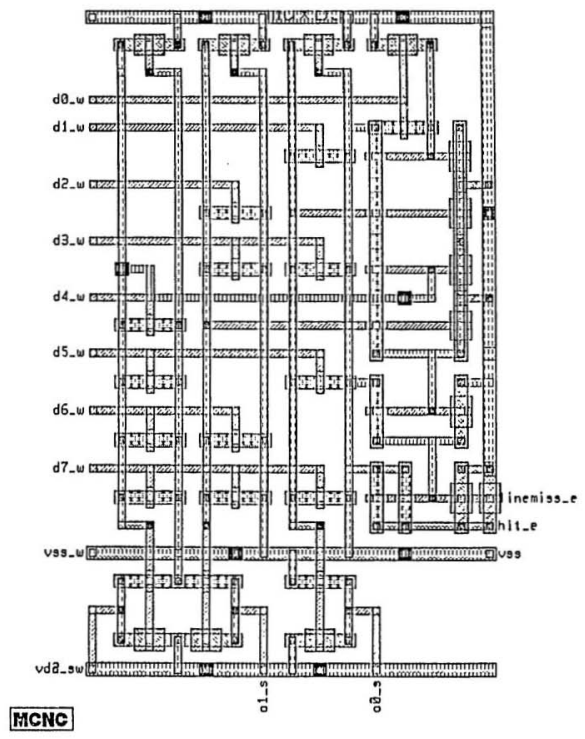

Figure 15: Layout of the line Numler Generator 


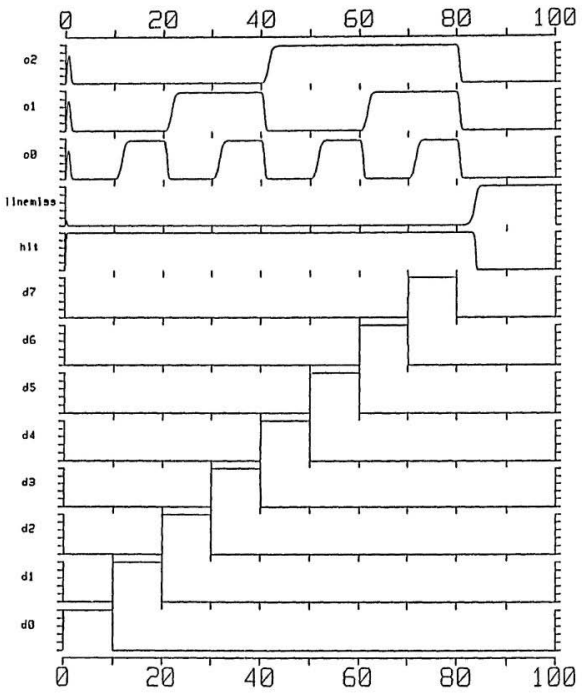

Figure 16: Simulation of the Line Number Generator 


\begin{tabular}{|c|c|c|c|c|c|c|c||c|c|c|}
\hline \multicolumn{9}{|c||}{ Ilot Code } & \multicolumn{3}{|c|}{ Binary Code } \\
\hline 0 & 0 & 0 & 0 & 0 & 0 & 0 & 0 & \multicolumn{3}{|c|}{ Line Miss } \\
\hline 0 & 0 & 0 & 0 & 0 & 0 & 0 & 1 & 0 & 0 & 0 \\
\hline 0 & 0 & 0 & 0 & 0 & 0 & 1 & 0 & 0 & 0 & 1 \\
\hline 0 & 0 & 0 & 0 & 0 & 1 & 0 & 0 & 0 & 1 & 0 \\
\hline 0 & 0 & 0 & 0 & 1 & 0 & 0 & 0 & 0 & 1 & 1 \\
\hline 0 & 0 & 0 & 1 & 0 & 0 & 0 & 0 & 1 & 0 & 0 \\
\hline 0 & 0 & 1 & 0 & 0 & 0 & 0 & 0 & 1 & 0 & 1 \\
\hline 0 & 1 & 0 & 0 & 0 & 0 & 0 & 0 & 1 & 1 & 0 \\
\hline 1 & 0 & 0 & 0 & 0 & 0 & 0 & 0 & 1 & 1 & 1 \\
\hline
\end{tabular}

Table 3: "The Truth Table Relating the "Hot Code" and the Binary Code

\begin{tabular}{|c|c|c|c|c|c|}
\hline \multicolumn{3}{|c|}{ Operations For A Linc llit } & \multicolumn{3}{|c|}{ Operations For $\Lambda$ Line Mliss } \\
\hline \multicolumn{2}{|c|}{ Component Name } & \multirow{2}{*}{\begin{tabular}{|c|} 
Delay 'lime(ns) \\
\end{tabular}} & \multicolumn{2}{|c|}{ Component Nane } & \multirow{2}{*}{$\frac{\text { Delay Time:(11: }}{1}$} \\
\hline Decoder & & & Deroder & & \\
\hline \multirow[t]{2}{*}{ Tag Array } & comp. & 3 & \multirow[t]{2}{*}{ T'ang Array } & comp. & 3 \\
\hline & updale & & & updatc & 6 \\
\hline \multirow[t]{2}{*}{ LRU } & sendback & & \multirow[t]{2}{*}{ LIRU } & sendback & 2 \\
\hline & update & 8 & & update & \\
\hline LNG & & 2 & LNG & & 4 \\
\hline 'Total & & 14 & Total & & 16 \\
\hline
\end{tabular}

Table 4: Time Delay for the Directory

Tag Array[comp.] + LNG + LRU [update]). Since the updating operations of the

LRU unit can overlap with the directory search for next read/worite operation (note:

that the cache is pipclined), the actual delay can be less than calculated above. If

there is a line miss, the total delay is at least 16ns (Decoder + Ting Array[comp.]

+2 LNG + LRU[senclback] + Tag Array[updatc] $)$. 


\subsection{The Line Replacement Unit}

In a cache system, one of the related problems is to predict which sets of addresses already buflered in the cache memory will be needed furthest in the future becruse it would then be possible to determine the optinum line to be replaced by a new one from the main memory. Since this algorithin is based on future knowledge of the program's beltavior, it cannot be realized in a practical cache memory. Therefore, some approximation must be made to this ideal. In the cache system described here, the least recently used line replacement (LRU) algorithm is employed. Under this strategy, the line to which any memory references were made the longest time ago is replaced by a new one. This algorithm is based on the assumption that the line which was referenced the longest time ago is the most likely not to be used in the near future; it relies on the temporal locality of reference characteristic of most programs.

The unil shown in Fig. 17 consists of 32 LRU cells which can be selected individually by the 32 -tit dircetory decoder. It is organized into four rows, with 8 LRU cells per row. During initialization, the $\overline{R E S}$ signal resets all the LRU cells.

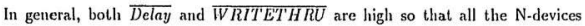
connecting to these signals are closed while all the P-devices are open. Under this condition, the III' signal can be propagated through the $\mathrm{N}$-devices directly to affect all the $I I T^{\prime}(d)$ 's. If there is a signal $I I T T^{\prime}$ from the line number generator after searching the directory during a read/write operation (the requested data reside in the cache), one of the $32 \mathrm{LRU}$ cells selected by the $32-6$ it decoder is updated by 


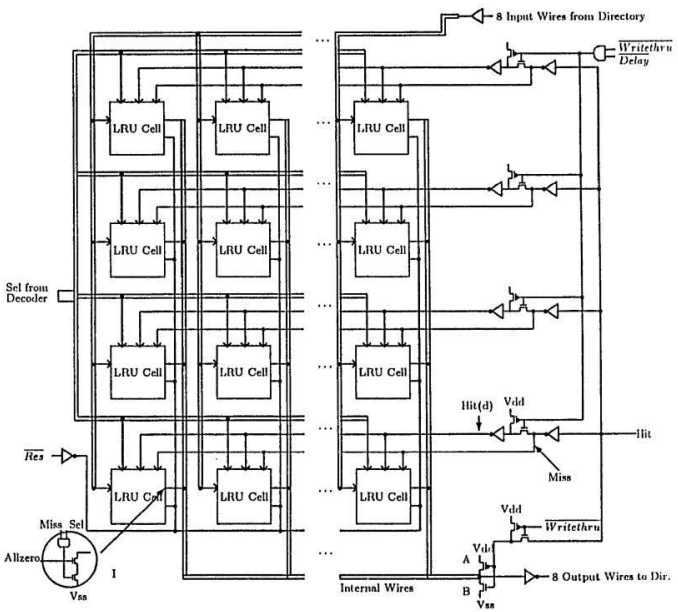

Figure 17: Structure of the LRU Unit 
8 signals from the directory, ench one corresponding to one line number slot of a given set. (The outputs of this LRU cell are locked by a low MISS.) Meanwhile, the IITT signal also passes the nNOS pits-transistor to gates of both devices $A$ and B since WRITETHRU is high. The internal wires connecting the outputs of 8 pairs of devices $\mathrm{A}$ and $\mathrm{B}$ to the outputs of the LRU cells remain low regardless of the status of ALLZERO's shown in subfigure I of Fig. 17 since the N-devices B are closed to discharge the wires, making all 8 output wires to the directory at high level after the inverters. Tlus, the directory can be prevented from updating. If IIIT becones low after the directory is searched (that means a line miss occurs), the MIISS signal is high so that the LRU cell selected by the directory decoder can send the least recently used line slot number of a given set to the directory while the corresponding $\operatorname{IIT}(d)$ signal is low to prevent the LRU cell from updating. Meanwhile, the low signal IIIT switches devices A on and $\mathbf{B}$ off through the $\mathrm{N}$-pass transistor controlled by $\overline{W^{\prime} R T E T M R U}$. The 8 internal wires are charged by devices A immediately. These wires are connected to one LRU cell selected by SEL from the tecoder. As shown in subfignre I, the transistors controlled by the result of ANDing the signals MISS and SEL are now closed so that the 8 internal wires can be used to evaluate values on the 8 ALLZERO's of the given LRU cell. Only one of $8 \overline{A L L Z E K O}$ 's in the LRU cell is low to indicate that the corresponding line is the least recently uscd line in the selected set of the cache. So, the corresponding wire is charged high while other wires are held low by passing charge to ground through the two cascacled HNOS pass-t ransistors in subfigure I. 'The: active (high) 
internal wire is inverted to update the associated line slot, and the remaining keep their line slots in the given set of the directory unchanged. Note that transisturs $A$ and $B$ are balanced with those shown in subfigure I so that the operations are reliably completed in minimum time.

In Fig. 17, it is seen that if $\overline{D E L A Y}$ is low, the unit is prevented from upelating by cutting of the HIT signal. Also if WRITETIIRU becoms low, the unit is prevented from both updating itself and changing line slots in the directory by

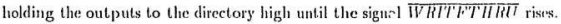

DEELAY is designed to handle the situation that, during a read/write operation, the LRU unit may otherwise be upelated incorrectly before the directory turns ont a valid result for $/ 1 \mathrm{IT}$. This is hecause at the fueginning of an operation the directory decoder selects both a given LRU ceil and a corresponding row of the tag arraty: During the period that the tag army searchs for a lite slot in the specilied row in which the requested address resides (the line nembre generator has not turned wut a valid HIT for this operation atud the signal IIIT stands at a high tevel at this time), the status of the IIRU cell may be changed by the invalict signal IIIT. Fior instance, assuming that, before ALE is assertem, the output of the 32-bit dermer $r$ is 8 and IIIT is logical 1. When ALE' is asserted, a new address whose sph number is 10 is lateled in the address register and broadeast to the decoder immerliately: 'The. oulput of the decoder (here it is 10) is sent simultantously to both the tang array; to see if the requested data reside in the cache, and the LRII unit, either to updalethe corresponding LRU cell if the requested dat a are in the rorrespontling sot, or Lo 
send the lcast receutly used line slot number to the directory if the requested data are missing. Because searching the directory takes more time than propagating the signal from the 32-bit decoder to the LRU unit, the LRU cell corresponding to set 10 is updated before the line number generator turns out a new result for IIIT since the old $H \mathrm{HI}$ ' still remains effectively at logical $\mathbf{1}$. This results in an error! The signal $\overline{D E L A Y}$ is used to prevent the LRU unit from being updated.

As slown in Fig. 17, when $\overline{D E L A Y}$ is high, all the N-pass transistors connected to DE:LAY through an AND-gate are closed, while the P-pass transistors are open, to maintain all the $I I T(d)$ 's the sane as the signal HIT from the line number genervilar. Once DELAY becomes low, the N-type devices are open and the pathes to $H I T^{\prime}(d)$ 's are cut off while MISS's remain as $\overline{I T T}$. Meanwhile, the P-type devices are closel to charge the inverters so that $I I T(d)$ 's are discharged via the inverters to prevent the LiRU cells from being updated. After a period during which the line number generntor produces a valid result, $\overline{D E L A Y}$ changes to logical 1 so that the LRU unit can correctly operate depending on the valid signal IIIT. T'Itus, correctly upclating of the LRU unit duting a read/write operation is cusured. The valid period of the signal $\overline{D E L A Y}$ is an inverted 4-nanosecond pulse, w! ich is long 'nough for a senteh of the directory and a valid result of the IIIT signal to be cone stable.

The $\overline{D F L A Y}$ sigual can be produced by an one-shot circuit, as shovn in Fig. 18 , which can protuce a narrow pulse from a wide pulse. When the input $V_{\text {, of }}$ the rircuit is imposed logical 0 , the inverter precharges capacitor $\mathrm{C}$ through resistor 


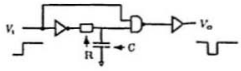

Figure 18: The One-shot Circuit

R. Meanwhile, because the 2-input NAND-gate is locked by $V_{i}$, the output of the NAND-gate remains ligh so that the output $V_{0}$ is high. Whenever $V_{1}$ is changed from 0 to 1 , since the NAND.gate input connecting to the capacitor still remains high, the citput of the NAND gate causes $V_{o}$ to becone low inmerliately. After the capacitor $\mathrm{C}$ is discharged below the threshold of the NANI)-gate, the output of the NAND-gate becomes high regardless of $V_{i}$ so that the output $V_{o}$ is pulled up to logical 1 by the buffer. The period of the inverted pulse of the circuit output is determined by the RC (resistance-capacitance) product. Resistor R is inplemu'nted by a polysilicon wire while capacitor $\mathrm{C}$ is formed by an $\mathrm{N}$-devire gate. In order to produce the $\overline{D E L A Y}$ signal, $A L E$ is imposed on $V_{i}$, and the output of this circuit is a 4-nanosecond pulse of DELAY.

The WRITETHRI signal is used to ensure that the status of the l,RU unit remains unclanged during an upelate operation required by other caches in a mul. tiprocessor environment. That is, whenever there is an update request from other caches, the cache must not change the contents of both the directory (if the datit to be updated are not found in the cache) and the LIRU unit (if the datia are in the 
(achele). For an upelate operation, if the requested data are found in the cache, the cache only uprlates the data without changing records in the LRU unit; otherwise, the cache does nothing for this request. IIence, a signal WRITETIIRU from the miss circuit is used to landle this situation. Whenever there is an updite request, the miss circuil produces the WRI'ETHRU signal. The signal WRITETIIRU locks the HIT signal to prevent records in the LRU unit from modification. This operation is similar to that of $\overline{D E L A Y}$. Meanwhile, it is also used to lock the path to the directory to prevent the dircetory from danging if the requested datit are: not found in the cache. That is, during an update request, the IVRTTETIITU signal becones low to lock the uMOS pass transistor and hurn on the P-Lype dovice to charge the gates of transistors $A$ and $B$. 'Thus, P-devices $A$ are opened while $\mathrm{N}$-devices $\mathrm{B}$ are closed to discharge the 8 internal wires so that the outputs to the directory remain ligh to prevent the directory from being modified during WRTETHRU.

The output structure of the LRU unit is organized in this way because of the ontput delay time. Since the interual wire propagation delays caused by the distributed resistance-capacitance product are large and the capacitive load on cach wire is heavy (each wire is connected to all the 32 LRU colls), simulations show that the use of standard (MOS design tedniques can not obtain high speed. 


\subsubsection{One LRU Cell}

Fig. 19 shows one of $32 \mathrm{LRU}$ cells which is selected by the 32-bit decoter. Each cell corresponds to a set of the cache. An LIRU cell is an $8 \times 8$ binary matrix in which there are no bits on the diagonal. Both the row number and the column number represent the line number (indieated by $l_{1}$ to $l_{7}$ from the directory in Fig. 19) in the seb selected by the 32-bit decoder. Bach matrix corresponds to a set in the directory. Changes in the state of a matrix are controlled by the uplate control circuit of the LIRU cell. When IIIT(d) from the directory is high, the requested line resides in the set selected by $S E L$ from the decoder. The control circuit simultancously updates the statcs of the row and the columu indicated by the line number. Assuning that the ith line in the selected set is requested and the line is in the cache, all the bits of the ith row of the corresponding matrix arr changed to 1 to record the fact that the corresponding line was the last one used while all the bits in the ith column of the matrix are reset to zoros to clecrense the number of l's of other rows: The number of I's in a row represents the used times for the corresponding line. The largest number, all-oue's, indicates that the corresponding line is the most recently used while the smallest mumber, all-zero's, is the least recently used. In cach row, $\tau$ bits are compared. If and only if all the. bits in a row are reseb to logical 0, the corresponding ROVAIATC'I/ line: is high.

In turn it makes the corresponding signal $\overline{A l, L Z E W O}$ of this row low, when tuth $S E L$ from the 32-bit decoder and MISSS from the line number gencrator are logingl 1, while 7 other $\overline{A L I . Z E K O}$ 's remain high. 


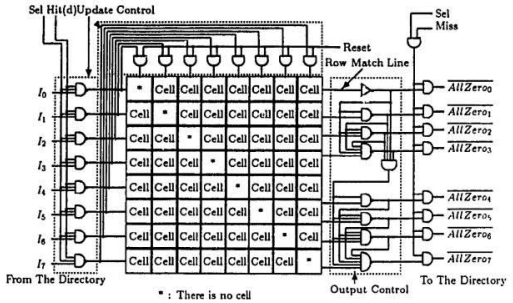

Figure 19: Structure of an LRU Cell

For example, Fig. 20 shows an LRU cell which is a $4 \times 4$ matrix. Both the mws and the columns are numbered from 0 to 3 , which represent the line numbers. The initial status is shown in Fig. 20 (a); line 1 is the most recently used one since the number (here it is 3 ) of 1 's in row 1 of the binary matrix is the largest while line 0 is the least recently used one because the first row is all-zeroes. After teference to line 2, the matrix is updated as shown in Fig. 20 (b), line 2 becomes the most recently used one by setting row 2 full of ones while line 0 still remains at the last. position in order after resetting the column 2 full of zeroes. Similarly in Fig. 20 (c), after reference to line 0 , the order of the lines changes to $0,2,1$ and 3 . Now line 0 becomes the most recently used one after row 0 is set full of ones while line 3 


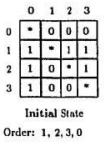

(a)

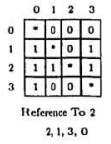

(b)

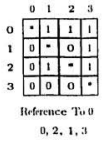

(11)

Figure 20: An Example of the LRU Algorithı!

bccomes all-zcroes after column 0 is written full of zerocs to dectrass the unuluers of all the rows except row 0 .

It is clear that the least recently used line in the selected set of the carlere is lhe one for which the row is entirdy equal to 0 and the colmun is contirely equal to l. Therefore, if a line miss occurs in that set, the LRU cell specified ly both, SE I, ant MISS sends the least recently used line slot number to the directory. In sone ("aines more than one row can be: all-zeroes, for example, initially all the rows alte wave after RESET'. This means that not all the lines in the selected set, of the ciachu' memory are full. In this situation, the output control which is composed of . X.X.1) gates in Fig. 19 is used to pass the line whose number is the smallest of all ileunused lines in the set as the least recently used line by pulling dewn its unt puil $\overline{\text { ALLZERO }}$ and to lock other unnsed lines by maintaining Ilerir correspenulium, ALIZERO's high. Whenever an output is low (meaning that the line slot number implied by this bit is the least recently used line slot number in the gives se+t), it 
locks all other out.puts which follow by NANDing all the ROWMATCII signals logically behind it. Fig. 21 shows the layout of an LRU coll.

\subsubsection{One Bit of the LRU Cell}

The logic of one bit of the LIUU cell is illustrated in Fig. 22. The bit cell used in the LRU cell is a variation of a static memory bit. If a signal imposed on ROWSEL is high, inverter $B$ is pulled down to 0 while inverter $A$ is brought up to 1 . Thus, the MATCII signal becomes low by closing the MATCII transistor. On the other hand, if the COLSEL line is asserted, the A inverter becomes low and B is high. 'The MATCll transistor is open so that the signal MATCH of this bit is high. Note that the signals ROWSEL and COLSEL must not be asserted at the same time, to prevent this bit from being placed in an indeterninate state. In an LRU cell, all the MATCII lines and all the ROWSEL lines of the LRU bits in a row are comected in series, respectively, and all the COLSEL lines of the bits in a column are commected in serics. A row can be selected by $I_{i}$ from the directory when both $I I I^{\prime}$ ' and $S E L$ are high. If all the $M A T C H$ lines of the bits in a row are high, implying that all the bits in the row are at logical 0 , the ROWMATCII line of this row becones high to indicate that this row has been set to all-zeros. The carhe line corresponding to this row may be the least recently used line (the least recently used line in a given set is inclicated by a low ALLZERO signal of the given LRU rell), tepending on whether any of the rows logically before this one have high ROIVMATCHes. 


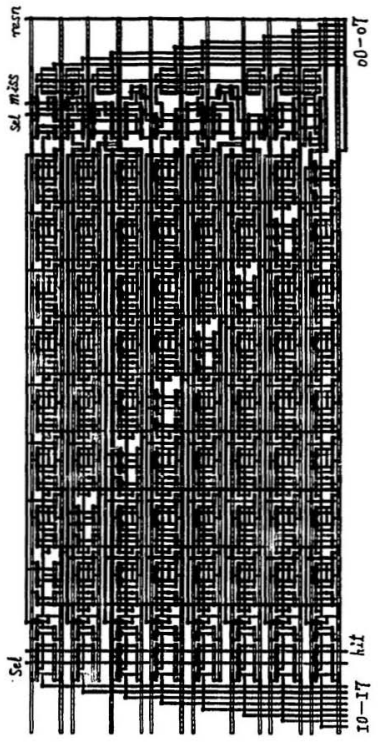

Figure 21: bayout of the LRU Cell 


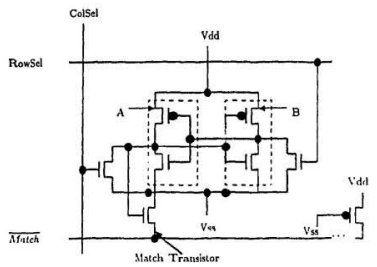

Figure 22: One Bit of the LRU Cell

Fig. 23 shows a simulation result of the LRU unit. The signals from $I_{0}$ to $I$ - art 8 input lines from the directory and $O_{0}$ to $O_{7}$ are $\&$ output lines to th $_{1}$ - directory. After the signal RESN is valid (low), the unit is reset. It can be sern in thr simulation in Fig. 23 that all the LRU unit outputs $O_{1}$ to $O_{7}$ are high exrept $O_{1}$. This neans the cache line implicd by the low $O_{0}$ outpnt is the least recently used line after initinlization, aithough at this time all the rows in a giren cell are zorrus. since the first row indicates the smallest line number in the given set. When IIIT is logical 1 (note that the low HIT signal implies that . HISS is high) and $I_{0}$ is high, the first row of a given LRU cell is updated to all-ones. At this : inw. Ilime are no ontputs on the output lines (from $O_{0}$ to $O_{i}$ ) of the LRU whit, which indicit" that a line miss occurs. During .MISS, the outputs of the LRE mit. are : alir'. In 


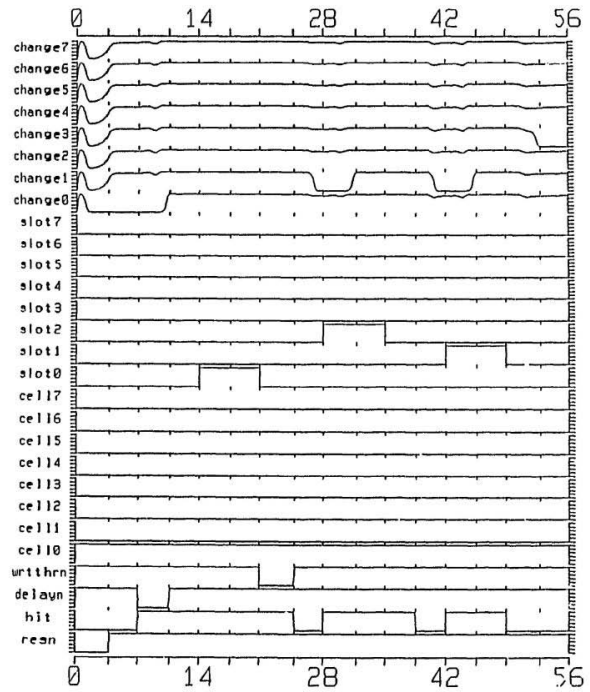

Figure 23: Simuiation of the l, ILU Unit 
this casc, $O_{1}$ becomes low, which indicates the first row has been updated and the line indicated by the second row in the given cell is the least recently usel line. After the $3 \mathrm{rd}$ row is updated to all-ones during $I_{2}$, when MISS is asscrted again, the least recently used line is still the line indicated by the second row. It can be seen that at the second MISS the LRU unit produces a low output at $O_{1}$. T'hen after updating the second row by asserting $I_{1}$, the LRU unit produces the least recently used line, during the next $M I S S$, implied by the 4th row whose ontput is $O_{3}$. From the simulation we can see that the delay time for a valid output is about 3 nanoseconds. Note that the signal $D E L A Y N$ in the simulation is used as the result of ANDing $\overline{D E L A Y}$ and $\overline{\text { WRITETHRU }}$ to prevent the LRU unit from being updated.

In this chapter, cache algorithms are surveyed. CMOS implementations of algorithms selected for this cache design such as the 8-way set-associative mapping and the lenst recently used algoriblims are discussed. A bit-matrix method is userd to simplify the implementation of the LRU algorithm in CMOS. The functions of the Directory and the LRU Unit have been verified by circuit simulation. 


\section{THE MEMORY AND CONTROL UNITS}

One of the most important parts in a caclie is the cache buffer, or data storage, which is used to store the most up-to-date data. Its main function is similar to that of a small, high speed random access memory. Another is the control unit which determines the internal and external timing of the cache, controls the functions implemented in the previous chapter, and provides the communication functions required for a multiprocessor or uniprocessor environment. This chapter discusses the design and implementation of both the cache buffer and the control unit used in this project.

\subsection{Structure of the Menory}

Fig. 24 shows the structure of the cache memory in which a row represents onc line, with 8 words per line and 32 bits in each word. The row number is in the range 0 to 255 . An 8 -word line size was chosen for this cache memory, assuming that the associated main menory is partitioned into 8 modules which can transfer a requesied line to the cache by interleaving. This main memory organization is more suitable for a multiprocessor systcm.

A cache memory address is divided into two parts; one part containing the set and line numbers is used to select the specific line in the cache memory through the memory decoder while another part (the offset) in the register/counter is usid to deternine which word or byte(s) in the selected line is (are) accessed. 


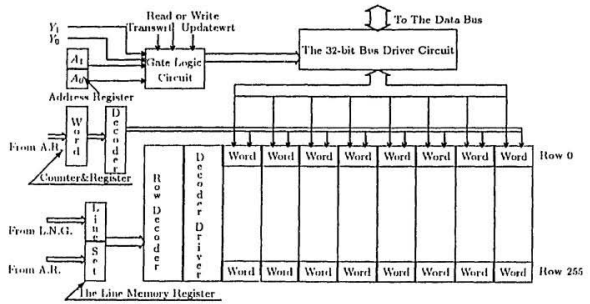

Figure 24: Structure of the Cache Memory

In this system, the smallest element the processor can access is not a word but at byte. There are 4 sizes of data which can directly be accessed by the processor -- one, two, three or four byte blocks. T'he size of the data to be accessed by the processor is determined by a combination of the two least significant bits of the requested data address, $A_{0}$ and $A_{1}$, and the two function bits, $Y_{1}$ and $Y_{2}$, which come from the processor. 1)uring a processor real/write operation, when the requested data reside in the cache memory, the cache will either send the requested dala to the data bus or store the data on the data bus into the cache, depending on the specific operation of the processor. In this case, the register/counter performs like a register. The word oflset of the address register is latched in this special register, and the recuested word is selected via the column decoler of the memory. Otherwise, the miss flag is set to indicate that the requested data are not in the 


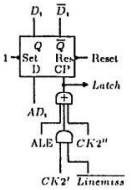

Figure 25: One Bil. of the Mennory liegister

cache. During a line miss, the least recently used line in a given set of the cache memory is overwritten witl, the requested line from the main memory. In this case, the register/counter becomes a counter, controlled by the TRANSFER signal from the minin menory, to elioose each of the 8 possible wort offsets in the selected line in the increasing order (from 0 to 7). For earch time at which a word olfset is chosen, one of the 8 words in the requested line from the main memory is written to the corresponding location. The main memory sends 8 words of the requested line, by interleaving, for a miss recuest. Fach word being transferred is accompanied by a valid pulse of the TRANSWRT signal, which is used to increment the connter and to require the bus control cirevil to pass a word on the data bus to the caclue memory during transfer.

\subsubsection{The Caclie Memory Register}

The cache memory register is composed of two parts: one is the line memory register and the other is the counter/register. Pig. 25 illustrates one bit of the line 
memory register which is used for both the line number and the set number of the cache menory. $A \mathrm{D}$-type flip-flop is employed. The signal for latehing a cache menory address into the memory register, including both the cache line register and the register/counter, is also described in this figure. Latehing a cache memory address in the mrmory rrgisier occurs under two conditions. The first condition is that during a read/write operation, the corresponding cache memory address has to be latehed in this register after senrehing the directory. If this condition is satisfied, the $A L E^{\prime}$ signal produced by the clock pelse generntor is asserted. The' second condition is that when there is an upelate recpuest from othre cacles and the requested clata are found in the cache, the clork pulse generator produces two pulses $C K 2^{\prime}$ and $C K^{\prime} 2^{\prime \prime}$ for this request. 'The $C K^{\prime} 2^{\prime}$ signal is used to lateh the cauche address corresponding to the requested data during the update operation. After the operation, $\mathrm{C}^{\prime} 2^{\prime \prime}$ returns the address residing in the memory register before the operation. Whether or not the requested data reside in the cache is indicated by the LINEMISS signal from the line number generator, after searching the directory during the update request. 'Thus C $K 2^{\prime}$ has to be ORed with LINEMIS'S to fortn the latching signal for the upclate operation. Note that LINEMISS is produced by the line number gencrutor. It differs from the NISS signal from the miss flag which is used to inform the main memory "rat the data requested by the associated processor are not in the cache.

The logic circuit for the counter/register is illustrated in Fig. 26. It consists of 3 D-type falling-edge-triggered flip-flops which are organized as a synchronous 


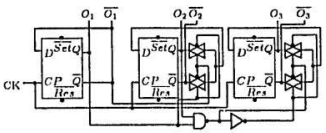

(a) Struct ure of The Syartarouous Counter

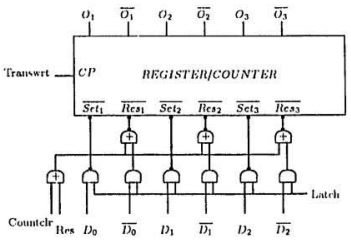

(b) Functional Hlor-k l)ingram of Tlu- C'ounter/Hegister

Figure 26: 'The Register/Counter Logical Cirenit. 
counter slown in Pig. 26 (a), but it, can operate as both a counter and a register in different siluations. The logic function block for this circuil is depicted in Fig. 20 (b). The intializing signal, system RESET or COUNTCLR from the transfcr decomposer, resets this circuit. The word offset of an address on circuit inputs $D_{0}-D_{2}$ and $\overline{D_{0}}-\overline{D_{2}}$ can be latrhed from SET's and RES's of the D-type flip. Hops, respectively, when the LATC'/I signal is active; LAT' $/ /$ is created when Lhere is either a hit, during a read/write operation, or an update recuest from other caches. 'The 3 -bit outputs $\mathrm{O}_{0}-\mathrm{O}_{2}$ and $\overline{\mathrm{O}_{0}}-\overline{\mathrm{O}_{2}}$ arc sent to an 8 - hit memory colum decoder implenented with cight 3-input NOR-gates, in a way similar to the 16-bit decoler described previously. When there is a miss, the low LATC'II signal locks the data inputs of this circuit and TRANSWRT, from the transfer decomposer; is imposed on CK of the comter after COUNTCLR resets the counter/register: At this time, the counter/register behaves like a counter. When each pulse of the TRANSIVRT signal is imposed on $C K$ of the counter, the outputs of the counter are not changed until the falling edge of the pulse. Thus, when a word is transferted into the memory during each pulse of the TRANSWRT signal, the corresponding word offset selected by the memory column decoder cannot be changed, which guarantes 8 words of the requested line are transferred into correct places in the memory. 'The layout of the memory column control, including the counter/register and 8-bit column decoter, is illustrated in Fig. 27 and its simulation is shown in Fig. 28.

'The circuit of the transfer decomposer is shown in Fig. 29 (a). Since the number 


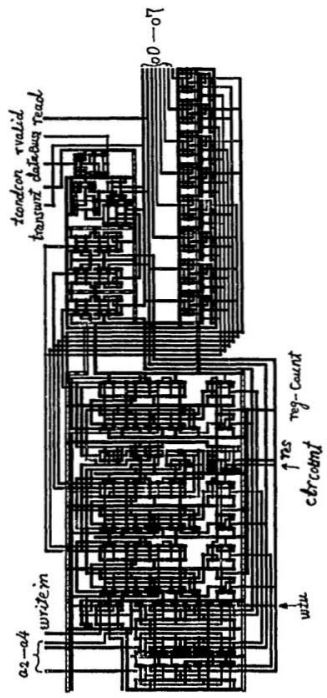

Figure 27: Layout of the Me:mory Columu Control 


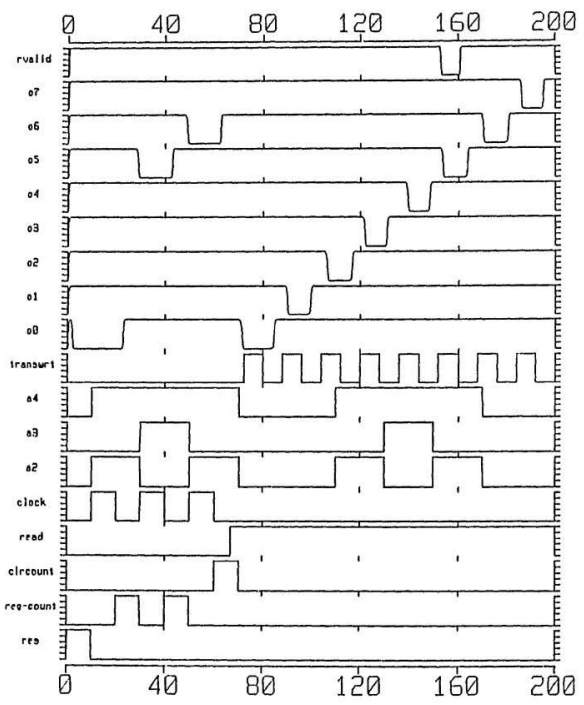

Figure 28: Simulation of the Menory Column Control 


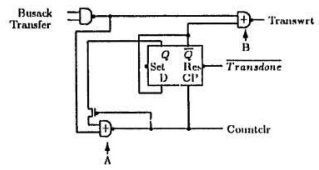

(n) The Cireuit of the Line Tranefer For Miess

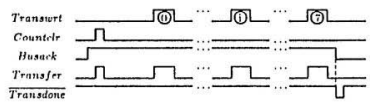

(b) The Tinuing Dingram of The Line T'ransfer For Mise

Figure 29: Circuit of the Transfer Decomposer

of pits on this chip is limited, the TRANSFER signal from the main memory consists of two parts: the first narrow pulse is used to clear the counter, and the following 8 wide pulses are used to write a line on the clata bus into the cache memory, one word per pulse. 'The decomposer divides the TRANSFER signal into COUNTCLR which clears the counter and TRANSW RT which drives the counter from 0 to 7 and the the bus driving circuil during a lus grant indicated by the BUSACK from the system bus controller. Initially, the D-type falling-cdgetriggered flip-flop is reset so that its output $\bar{Q}$ is logical $1 . \bar{Q}$ is fed back to the $D$ input of the flip-flop. When the first pulse of the TRANSFER signal (used for clearing the counter) enters the circuit, it is gated through NOR-gate $A$ to crente a 


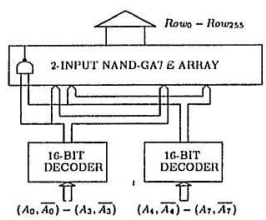

Pigure 30: 'The 256-bit Menory Decoder

valid COUNTCLR signal since the other input of the gate is logical 0 at this time, while TRANSWRT is invalid (low) because the 2-input NOR gate B is locked by $\bar{Q}$. When the falling edge of the first pulse of TRANSFER passes gate $A$, the flip-flop changes its state. $\Lambda$ transition from 0 to 1 of its output $Q$ locks NOR-gate A; meanwhile, NOR-gate B becomes unlocked to allow the following pulses of the T'RANSFER signal to get through NOR-gate B to the output TRANSWRT. After transfer of a requested line from the main memory to the cache memory, the cache control unit, produces an inverted pulse $\overline{T R A N S D O N E}$ to reset the D flipflop at the transition of $\overline{B U S A C K}$ from 0 to 1. Fig. 29 (b) is the timing diagram for operations of the transfer decomposer during the transfer of a requested line from main memory. 


\subsubsection{The 256-bit Row Decoder}

This 256-bit decoder is used to decode 8 bits of both the set number and line number from the memory register simultaneously, 5 bits for the set number and 3 bits for the line number. It can produce 256-bit outputs, but only one bit of all the outputs is logical 0 at any given lime, to be used to select one out of 256 rows of the caclie memory. The ontputs of the decoder are connected to the invertel row drivers of the memory. The decoder consists of two 16-bit decoders discussed previously and 256 2-inpul NAND-gates as slown in Fig. 30. The simulation for the 256-bit decoder in Fig. 31 only shows the first 32 outputs of this decoder. l'rom the simulation, there is about 2 nanosecond delay for the decoder stage.

\subsubsection{The Cache Memory}

A fast static menory is used in the cache memory unit rather than smaller but slower dynamic memory, which also needs to be refreshed. Fig. 32 depicts the organization of the memory. It is split into four memory arrays, two array's in the upper row and two in the lower row. Both the upper row and lower row arrays are connected to outputs, $\overline{R O W S E L}$ 's, of the 256-bit row-decoder throught the inverted row drivers. Intermediate buffers are used between two memory arrays at the same row. When there is a $\overline{R O W S E L}$ signal active, $t$ wo arrays in the same row are selected simultaneously. Memory organized in this way cant reduce delay time and in turn increase the menory access speed. Fig. 33 shows any four adjoining memory bits in an array along with their column sclection circuits. The circuit for 

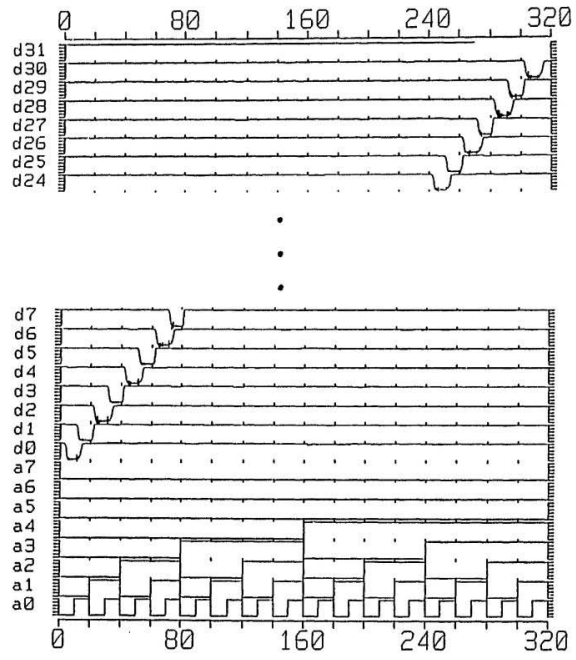

Figure 31: Simulalion of the 256-bit Row Decoter 


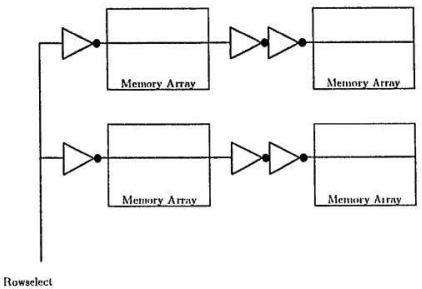

Figure 32: The Memory Arrays

column selection, one for cach columm of the memory, is çuitc simple; only two pass transistors are connected to the BI'T and $\overline{B I T}$ lines of memory cells in that column, respectively. Note that 32 column selections (one word) arc driven simultancously by one bit of the memory column docoder during access to one word. Therefore, a total of 256 column selections are formed as eight groups by connceting them to 8 output bits of the memory column decoder, respectively. Consequently, one memory array lias two groups, each containing one word. Only the cells selected by both row and column selections can be accessed. Fig. 33 shows four static CMOS RAM cells. Each RAM cell consists of two inverters wired logether to make a flipflop; they are connected by two nMOS pass transistors to the $B I^{\prime} T^{\prime}$ and $\overline{B I T}$ lines, respectively. During a read operation, the conducting side of the flip.-flop pulls the precharged data line $\left(B I^{\prime}\right.$ or $\left.\overline{B I T}\right)$ toward ground through the pass transistors 


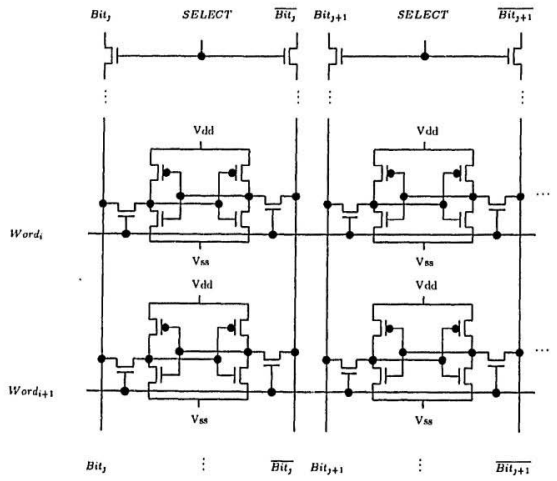

Figure 33: Four Bits of the Memory 


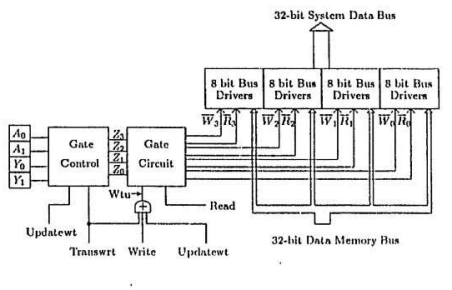

Figure 34: The Data Bus Control Circuit

while the other side remains high. Writing is accomplished by lorcing the value in the cell to be the same as that on the data lines.

\subsubsection{The Data Bus Control Circuit}

The data bus control circuit is shown in Fig. 34. The data bus driving circuit has 32-bit dual-port input/output drivers. It is split into 4 components, ench of which can control access to one byte. The opcration of each componcut is controlled by a pair of read and write control signals: $\left(\overline{R_{i}}, \overline{W_{i}}\right)$. There are four pairs of control siguals, $\left(\overline{R_{0}}, \overline{W_{0}}\right),\left(\overline{R_{1}}, \overline{W_{1}}\right),\left(\overline{R_{2}}, \overline{W_{2}}\right)$, and $\left(\overline{R_{3}}, \overline{W_{3}}\right)$ gencrated by the gate circuil.

As mentioned previously, data in the memory can be accessed as onc, lwo, three or four bytes, respectively, using combinations of $Y_{0}, Y_{1}, A_{0}$ and $A_{1}\left(A_{0}\right.$ and $A_{1}$ are in the two least significant bits, bit 0 and bit 1 , of the address register). During normal read/write operations of the processor, both TRANSWRT from 


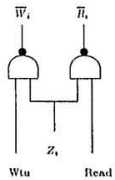

Figure 35: One Bit of the Gate Circuit,

\begin{tabular}{|c|c|c|c||c|c|c|c|}
\hline \multicolumn{3}{|c||}{ INPUT } & \multicolumn{5}{c|}{ OUTPUT } \\
\hline$Y_{1}$ & $Y_{0}$ & $A_{1}$ & $A_{0}$ & $Z_{3}$ & $Z_{2}$ & $Z_{1}$ & $Z_{0}$ \\
\hline $\mathbf{0}$ & 0 & 0 & 0 & 0 & 0 & 0 & 0 \\
\hline 0 & 0 & 0 & 1 & 0 & 0 & 0 & 0 \\
\hline 0 & 0 & 1 & 0 & 0 & 0 & 0 & 0 \\
\hline 0 & 0 & 1 & 1 & 0 & 0 & 0 & 0 \\
\hline 0 & 1 & 0 & 0 & 0 & 0 & 0 & 1 \\
\hline 0 & 1 & 0 & 1 & 0 & 0 & 1 & 0 \\
\hline 0 & 1 & 1 & 0 & 0 & 1 & 0 & 0 \\
\hline 0 & 1 & 0 & 1 & 1 & 0 & 0 & 0 \\
\hline 1 & 0 & 0 & 0 & 0 & 0 & 1 & 1 \\
\hline 1 & 0 & 0 & 1 & 0 & 1 & 1 & 0 \\
\hline 1 & 0 & 1 & 0 & 1 & 1 & 0 & 0 \\
\hline 1 & 0 & 1 & 1 & 0 & 0 & 0 & 0 \\
\hline 1 & 1 & 0 & 0 & 1 & 1 & 1 & 1 \\
\hline 1 & 1 & 0 & 1 & 0 & 1 & 1 & 1 \\
\hline 1 & 1 & 1 & 0 & 1 & 1 & 1 & 0 \\
\hline 1 & 1 & 1 & 1 & 0 & 0 & 0 & 0 \\
\hline
\end{tabular}

Table 5: The Gate Control Functions 
the transfor decomposer and UPDATEWRT produced by the cache control unit for updating data requested by other caches are not asserted (both of the signals TRANSWRT and UPDATEWRT' are logical 0$)$. Thercfore, signals $Z_{0}-Z_{3}$ produced by the gate control circuil dominate the read/wrile operations of the data bus control components via the gate circuil. There are four subcircuits producing $\bar{W}_{i}$ and $\bar{R}_{i}$ in the gatc circtit. Fig. 35 shows one subcircuit of the grte circuit. $\Lambda$ memory write operation is detcrmined by the condition that there is either a write operation required by the associated processor, a transfer operation during a line miss, or an update operation caused by an update request from other caches. If any of these operations is needed, the WTU signal from the 3-input OR-gate is asserted so that if any $Z_{i}$ is logical 1 , and the corrsponding write control bit $\bar{W}_{\mathrm{i}}$ is gated out to control an 8 . bit data bus driver to allow writing the data on the external data bus into the cache memory. If there is a read request from the processor, $\bar{R}_{i}$ is valid, causing the corresponding bus drivers to pass the recjuested data from the cache menory to the oulside data bus. Note that the operations of write and read are exclusive, so there is no case that $\bar{W}_{i}$ and $\overline{R_{i}}$ are assorted at the same time. 'The truth table for the signals $Z_{0}$ to $Z_{3}$ is shown in Table 5 .

After simplifying the functions spceified in Table 5 , we have the output functions, $Z_{0}-Z_{3}$, as follows:

$$
\begin{aligned}
& Z_{0}=\overline{A_{0} A_{1}} Y_{0}+\overline{A_{0} A_{1}} Y_{1}+\overline{A_{i}} Y_{0} Y_{1} \\
& Z_{1}=\overline{A_{1}} Y_{1}+\lambda_{0} \overline{A_{1}} Y_{0}+\overline{A_{0}} Y_{0} Y_{1}
\end{aligned}
$$




$$
\begin{aligned}
& Z_{2}=\overline{A_{1}} Y_{0} Y_{1}+A_{0} \bar{A}_{1} Y_{1}+\overline{A_{0}} A_{1} Y_{0}+\overline{A_{0}} A_{1} Y_{1} \\
& Z_{3}=\overline{A_{0}} Y_{0} Y_{1}+\bar{A}_{0} A_{1} Y_{1}+A_{0} A_{1} Y_{0} \overline{Y_{1}}
\end{aligned}
$$

These functions can be implemented efficiently by PLA. 'The logic circuit for the gate control functions implemonted in $\mathrm{Pl} A$ is illustrated in Fig. 36. The inputs to the circuit are the two least significant bits from the address register and two function bits, and their complements if needed. 'The outputs of the l'LA cirenit, $\overline{Z_{0}}$ to $\overline{Z_{3}}$, are NANDed with the result of NORing TRANSWRT' for transfer-write. of a requested line and UPDATE'WRT for update-write, to produce the ontputs $Z_{0}$ to $Z_{3}$. During a write operation both TRANSWRT and UPDATEW RT are at logical 0 so that the values of $Z_{0}$ to $Z_{3}$ are determined by the outputs of the PLA circuit. If either TRANSIVRT or UPDATEIVIT' is asserted, $Z_{0}$ to $Z_{3}$ are set high to force the bus drivers to overwrite one word ( 32 bits) on the system diata bus into the cache nemory. The layout of the gate control ind gate circuit is sliown in Fig. 37, and Fig. 38 shows the simulation for these circuits.

The data bus driving circuil is partitioned into four components, each for controlling 8 bits. A component is used to control 8 bits of the data to be written into or read out of the memory. Fig. 39 illustrates one bit of a component. There is a write logic block and a read logic block for cach bit of the data bus driver. The write logic is shown in Fig. 39 (a). When $\overline{W_{i}}$ is low and $W_{i}$ is high, the value on the $D A T A$ line is gated onto the BIT line and the $\overline{B I T}$ lines. Note that sizes of the transistors in this circuit are large enough to write datia into the memory at high speed. On the other hand, the read logic shown in lig. 39) (b) is more complicated 


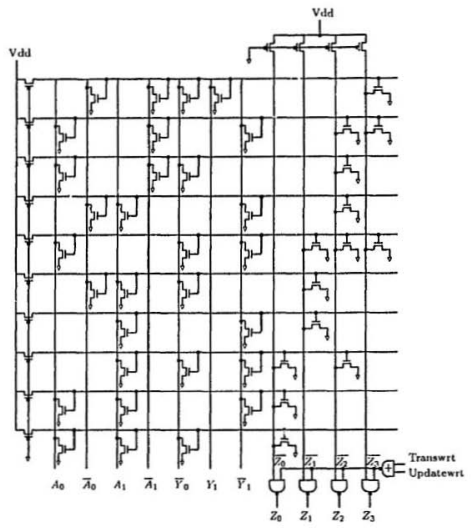

Figure 36: The Gale Control Logic 


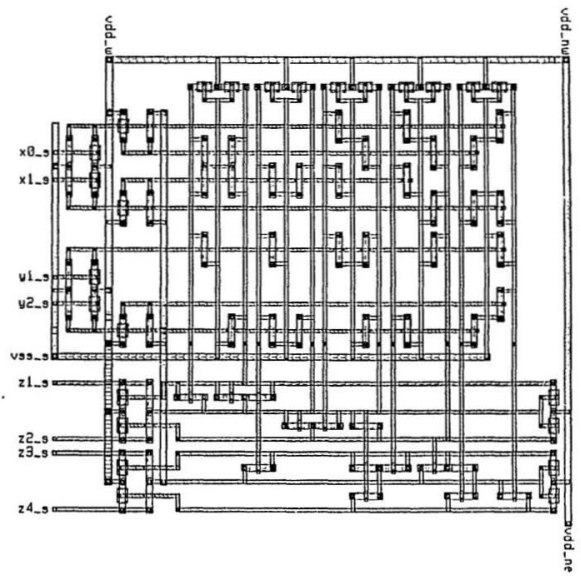

Figure 37: Layout of the Gate Logic 


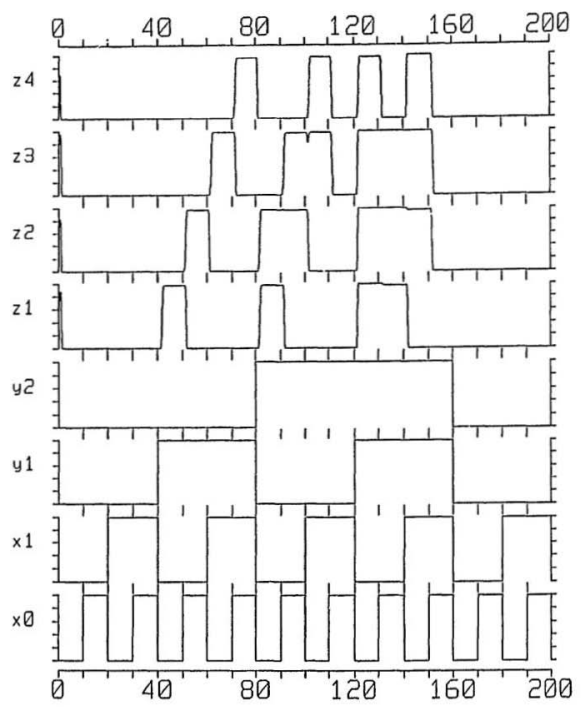

Figure 38: Simulation of the Gate Logic 


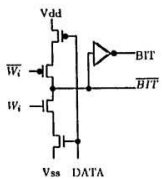

(a) Write Operntion Control

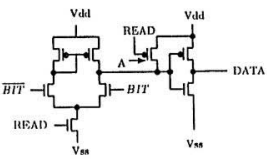

(b) Rend Operation Centrol

Figure 39: The Bus Write/Read Operation Control

since the signals from memory cells are usually weak. 'Therefore, there have to be amplifiers to increase the signal strength for the menory cells. This read circuit is a two-stage amplifier which detects the state of a memory cell. 'The first stage of the amplifier is a differential sense auplifier which can sense suall differences between voltage levels on the $B I T$ and $\overline{B I T}$ lines from the memory and amplify this to provide very fast sensing. The second stage is an inverter which provides further driving capacity and makes the rise time and fall time of the DATA signal shorter. Note that the differential sense amplifier is evaluated while the RIAI) signal is active. In order to obtain a correct output rapidly, the sense amplifier is precharged through transistor A to eliminate the charge-sharing effect during no read operations. Simulations for both the write and read logic control of a component are shown in Fig. 40 and Fig. 4J. In Fig. 40, when WRITE is high and READ is low, data on the DATA lines are gated to the BI'T' lines. 'The delay time is about 3 nanoseconds. In Fig. 41 , when IVRI'E' is low and $R E^{\prime} A D$ is high, data on the $B I T$ lines are quickly placed onto the $D A T$ ' $A$ lines. 


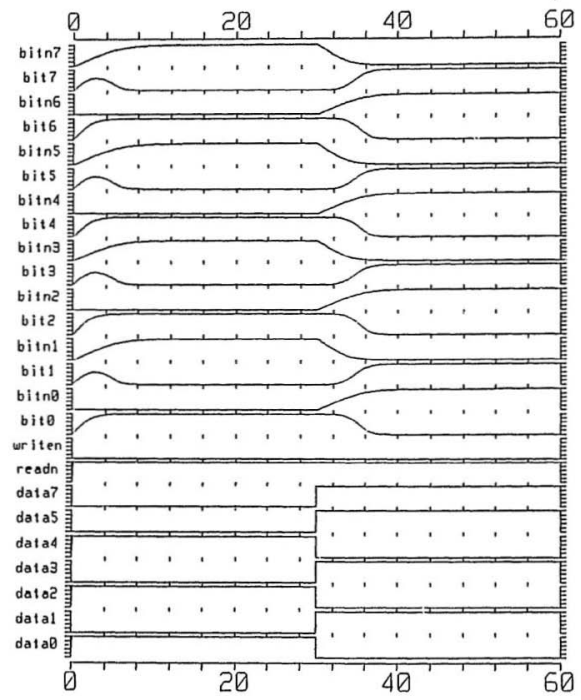

Figure 40: Simulation of the Write Control 


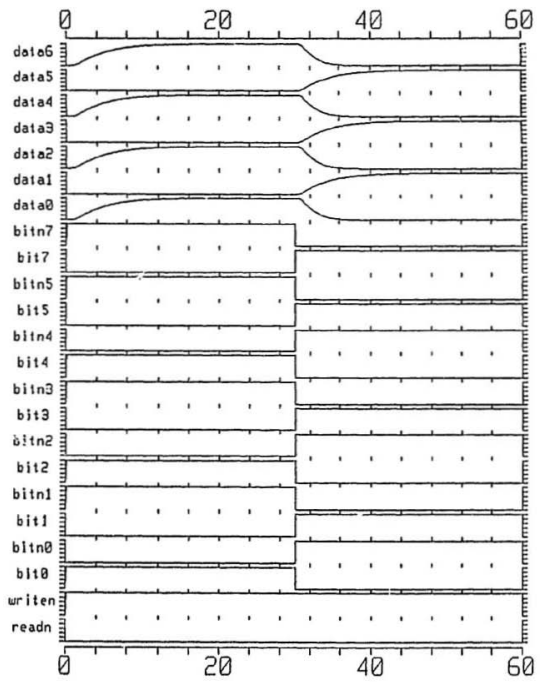

Figure 11: Simulation of the Read Control 


\subsection{The System Control Unit}

'The system control unit is used to control all the communications anong cache memory and the assoçiated processor, the main memory, as well as the multiple caches in a multiprocessor system. 'Therolore it is the most important part of this cache memory system. In terms of the communication operations, this unit is logically partitioned into three parts: the normal read/write operation, the update operation, and the miss operation. In this soction, more details about the functions of these lirec parts will be described.

\subsubsection{The Tiegular Read/Write Operations}

In order to control the clifferent operations, the clocks and $A L E$ thave to produce several clock pulses. A clock generator is used for this purpose. First, let us discuss the circuit in Fig. 42 (a), the single pulse producer. This circuit produces one complete pulse and its complement on the circuit onlputs $Q$ and $\bar{Q}$ from a sequence of pulses on $I N$ after receiving an inverted pulse on $\overline{C P}$. The belavior of this circuit is as follows: $\bar{Q}$ is initially at a high level since the 2-input NAND-gate input connecting to the flip-flop through nMOS pass-transistor $A$ is low regardless of $I N$. When there is an inverted pulse on $\overline{C P}$, the output of the flip-flop connected to pass trausistor $A$ is set lo logital 1 and it in turn cnables the 2-input NAND.gate since the pass transistor is closed by the high $\bar{Q}$ signal at this tinc. If $I N$ has a

transition from 0 to $1, \bar{Q}$ becomes low, and it in turn both locks pass transistor A and resets the flip-flop. Althongh the flip-flop is reset, the NAND-gate input 


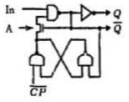

(a)

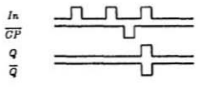

(b)

Figure 42: The Single Following Pulse Producer

connected to the pass transistor remains at logical 1 because the path to the input of the NANI)-gate is blocked by pass-transistor $\Lambda$. Therefore, the signal at. IN passes the NAND-gate to $Q$ and $\bar{Q}$. When the $I N$ signal makes a transition from 1 to 0 , the $\bar{Q}$ signal changes to logical 1 from logical 0 , which releases the pass transistor $\Lambda$ so that a low output from the llip-flop is imposed on the NAN1)-gat: input via transistor A. Thus the 2-input NAND-gate is locked so that the following pulses on $I N$ cannot be propagated to outputs $Q$ and $\bar{Q}$. The timing diagram in Fig. 42 (b) depicts the operation of this circuit.

Whenever there is an update request from other caches via the system bus, the update circuit produces an UPDATE signal to inform the cache to update with the data on the system bus. In this case, the clock generator produces several putses to accomplish the update operations. The circuit in Fig. 43 (a) is used to produce a pair of pulses $C K^{\prime} 1^{\prime}$ and $\overline{C K 1^{\prime}}$ from $C K 1$. At the beginning of $C K 1$, the circuit always checks if there is an UPDATE vignal. If the UPDATE signal is fount to be low after this check, $C K^{\prime} 1^{\prime}$ is set low while $\overline{C K 1^{\prime}}$ is set high. Otherwise, the high signal UPDATE is locked by a P-type pass-transistor on the input of the NANI). 


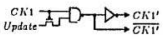

(a)

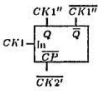

(b)

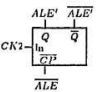

(e)

Figure 43: The Clock Pulse Generator

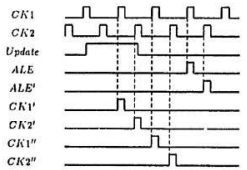

Figure 44: A 'l'ining Diagram for the Clock Pulse Gencrator 
gate so that during $C K 1$ any change of the UPDATE signal cannot allect the result. of the circuit, and $C K 1$ is gated out to create both $C K 1^{\prime}$ and $\overline{C K 1^{\prime}}$. Herce, even if $U P D A T E$ is changed during $C K 1$, the puise from $C K 1$ still passes the 2-inunt NAND-gate to make $C K 1^{\prime}$ and $\overline{C K 1^{\prime}}$ be complete pulses. Furthermore, the pulse generator also produces otlicr control pulses for an update operation using the pulse producers. Tirce pulse producers are cmployed to produce pairs of signals $\mathrm{CKI}^{\prime \prime}$ and $\overline{C K 1}^{\prime \prime}, C K 2^{\prime}$ and $\overline{C K 2^{\prime}}$, as weil as $C K 2^{\prime \prime}$ and $\overline{C K 2^{\prime \prime}}$ by imposing different signals on inputs of the circuits, as shown in Pig. 43 (b), (c), and (d), respectively. $C K 1^{\prime}$ is used to latch addresses from the system bus into the addrean register during an update request; $C K^{\prime} 2^{\prime}$ is to latch the corresponding cache addresses from the directory into the memory register to update the requested data. $C K 1^{\prime \prime}$ and $C K 2^{\prime \prime}$ are used to return the addresses which reside in both the address register and memory register before the update operation into the respective registers after the update. The pulse producer also generates an $A L E^{\prime}$ pulse, as shown in Fig: 43 (e), to latch a valid cache address from the directory into the memory register during a normal processor access. In order to produce $A L E^{y}, \overline{A L E}$ is imposed on $\overline{C P}$ of the pulse producer while $C K 2$ is present at $I N$. Fig. 44 shows a timing diagram for the mulse generator in which we can sec the relations among these signtals.

Note that all the inputs from the processor are controlled by the chip select $\overline{C S}$ signal from the associated processor. The cache memory can be accessed only when the cache is selected by the processor with $\overline{C S}$. The update operations, however, are not controlled by $\overline{C S}$. This makes the cache menory used in a cache-bascrl 
computer system more flexible.

\subsubsection{The Update Operations}

When the cache is used in a multiprocessor system, there must be a "bus watcher" to watch the system bus to sec if there are any other caclies reçucsting to update copies of data. The circuit in Fig. 45 is designed for this purpose. If there is an update request on the system bus, the bus update watcher must interrupt the caclie to update the data in the cache at the proper time. The proper time sliould be when either an access by the processor to the cache is finished or the cache is waiting for the system bus, in the cases of a write operation or a line miss. The finish of an access can be detected by a high $\overline{W / R}$ signal which can be obtained by NORing $W$ and $R$ from the processor. A request from the cache for use of the system bus can be made by BUSREQ from the bus control generator. Initially, nip-llop A and lip-flop B are reset when SEARCII is at logical 0 , and UPDATE from the bus update watcher is at logical 0 . (That means the circuit does not work as long as SEARCH is at logical O.) Flip-flop A is a R-S flip-flop while flip-flop B is a falling-edge-triggered D-type flip-flop. Whenever there is an update request from other caches, the SEARCHINT signal is gated in so that SEARCH becones high when BUSBUSY is low which indicates that the cache memory is not using the system bus. The output of the exclusive-or (XOR) gate has a level transition from 0 to I since the value of the XOR-gate input $F^{2}$ is logical 0 . The output of the XO12-gate passes through the 3-isput $\Lambda$ NI)-gate to both $C P$ of flip- 


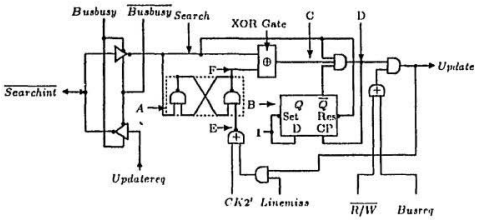

(a)



(b)

Figure 45: The Circuit of the Bus Update Watcher 
flop B and the 2-input AND-gate since the other inputs of the 3-input AND-gate (SEARCII and $\bar{Q}$ of flip-flop B) are at logical 1. Since flip-flop B is falling-edgetriggered, the state of the flip-flop is not changed at this time so that CP from flip-flop B is high. The result of the 3-input AND-gate is gated through the 2-input AND-gate to UPDATE if either a cache access is finished or the cache needs to use the system bus (that is, either $\overline{R / W}$ or BUSREQ is logical 1). Then, the UPDATE signal becomes valid. This mea.s the update operations can only be done when cither the cache memory is not used by its associated processor or the cache is waiting for use of the system bus. The UPDATE signal may be fed back to clear the R-S flip-flop in the case that the data to be updated are not found in the cache during the update operation, in which case LINEMISS from the line number generator becomes valid. In turn, a change of the XOR-gate from 1 to 0 triggers the D lip-flop since the $D$ of flip-flop $B$ is always logical 1 ( $\bar{Q}$ transits to logical 0 ); and this causes the UPDATE signal to become logical 0 . In this case, the cache does nothing since the data to be updated do not reside in the cache. If the ciata to be updated are in the cache (LINEMISS is low), flip?-flop A is not reset until $C K 2^{\prime}$ is asserted, then UPDATE is pulled low. When the SEARCHINT signal changes from 0 to 1 , both flip-flops are reset simultaneously, which means one update operation is finished. Fig. 45 (a) also depicts a dualdirection switch which can either gate in an update request on SEAIRCIINT from ollier caches, or gate out an update request on UPDATE'REQ from the bus control generator to other caches via the system bus. The dual-direction switch 
consists of two transmission gates controlled by BUSBUSY and $\overline{B U S B U S Y}$ from the bus control generator. When BUSBUSY is high, UPDATEREQ passes one of transmission gales onto $\frac{1}{S E A R C I I I N T}$ while the other transmission gate locks the path between $\overline{S E A R C H I N T}$ and SEARCH. When BUSBUSY is low, the signal on SEARCHINT from the system bus passes the gate to SEARCII while the path to UPDATEREQ is becked by the BUSBUSY' lincs. Fig. 45 (b) shows a tining diagram for the bus update watcher. From the diagran we can see the operation of this cirenit for two cases: one when no updating occurs and the other when updating occurs. In the first case, the data to be updatcod are not in the cache. in this case, the UPDATE' signal has a shorter valid period and is reset to logical 0 by LINEMISS as shown in the timing diagram. In the second case, the data reside in the cache menory. In this case, the period of the UPDATE signal is longer and UPDATE is cleared by $C K 2^{\prime}$. The longer UPDAT'E signal is uscel to produce an UPDATEWRT signal for writing the data on the system bus into the cache memory.

The circuit in Fig. 46 (a) is used to produce the UPDATEW RT' sign.t whirh, overwrites the data to be upelated if the data reside in the cacles. $(' k 2$ ' latches the UPDATE signal into the rising edge-triggered Mip-flop if the UPDATE' signal has a long period. The UPDATEWRT' signal is locked for the duration of C'T'2' hy an inverter clocked by $\mathrm{CK}^{\prime} 2^{\prime}$, since the updating write has to wait until the '256-bit. memory decoder finishes its operation. Meanwhile, the DA' ACONTROL signal is sent off the chip to control the path to the system bus. $\overline{C h 2^{\prime \prime}}$ is used to reset 


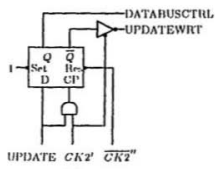

(a)



(b)

Figure 46: The Update-Write Generator

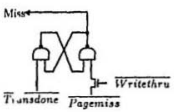

(a)

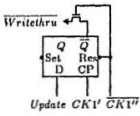

(b)

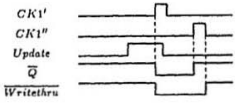

(c)

Figure 47: The Miss Circuit

the flip-flop and in turn UPDATEWRT. The timing diagram of this circuit in Fig. 46 (b) shows the operations described above.

\subsubsection{The Miss Operations}

As discussed previously, there is a circuit which create. a MISS signal when there is a line miss during a read/write operation. The MISS signal is produced by the miss circuit slown in Fig. 47. In Fig. 47 (a), initially, a high W $\overline{\text { ?ITETHRU }}$ signal forces the pass transistor to be closed so that the high-level $\overline{L I N E M I S S}$ signal from the line number generator (which means $I I T$ is high) can be inposed 
on an input of the flip-flop while another input of the flip-flop connected to the transfer clear circuit remains high. At this time, the output MISS of the circuit. is low. If there is a $I I T$ caused by a regular operation from the associated processor and there are no update requests from other caches (in this case, WRITETIIIRU is high), the state of the flip-(lop remains unchanged. If there is a line miss, the LINEMISS line is low so that the flip-flop sets its output MISS high. The MISS signal is used to make a request that the use of the system bus be granted to transfer a missing line from the main memory, and in lurn the main memory responds to this signal by sending the requested line, along with t'ie TRANSFER signal, to the cache after the system bus arbiter grants the cache the use of the system bus by setting $\overline{B U S A C K}$ of that cache valid (logical 0 ). At this time TRANSDONE from the tranfer clear circuit still remains high until $\overline{B U S A C K}$ makes a transition from 0 to 1 which indicates that the line transfer is finished. Then the transfer clear circuit produces the $\overline{T R A N S D O N E}$ signal, a narrow julse of about 4 nanoseconds, to reset the flip-flop so that the MISS signal changes from 1 to 0 .

As we have scen, the MISS signal is used to inform the main memory of a line miss. Note that not only a read/write operation can cause the line number generator to produce a LINEAISSS signal if the requested data are not found in the cache but also an update request from other caches in the multiprocessor system requires that a LINEMISS signal be generated. 'T'hese two kinds of line: misses must be handled in different ways. The way the first situation is landled 
has been discussed previously. For the second situation, absence of the data to be updated in the cache should not cause any changes in the cache. Therefore, in this case, the state of the miss flay should not be changed as a result of scarching the directory even though the search indicates the requested data do not reside in the cache. On other hand, if the data to be updated reside in the cache, the cache only updates the requested clata without changing the status of the cache. Thus, th :re has to be a circuit to ensure the cache contents are not changed during an update operation except for replacing the datat to be updated in the cache if the reçucsted data are found. In order to handle this case, the circuit shown in Fig. 47 (b) is cmployed to produce the $\overline{W R T E T I I R U}$ signal which prevents the miss flag and other components of the cache from changing during an update operation. For the miss flag, the WRITETIIRU signal is used to lock the path between the flip-llop input and $\overline{L I N E M I S S}$ to prevent the miss flag from changing the MISS signal by the $\overline{L I N E M I I S S}$ signal during an update operation. As discussed previously, $C K^{\prime} 1^{\prime}$ and $C K 2$ " are produced for an update opcration. $C K 1^{\prime}$ latches the address for the update into the address register and $C K 1^{\prime \prime}$ returns the address before updating into the address register. Hence, the WRITETIIRU signal must be at logical 0 to guarantee that the miss flag is not changed during the period between the begiuning of $C K 1^{\prime}$ and end of $C K 1^{\prime \prime}$. This circuit employs a rising-edge-triggered D Hip-flop. The flip-tlop is triggered by the rising edge of the $C K 1^{\prime}$ while UPDATE is high. $\overline{C K 1^{\prime \prime}}$ is used to clear the flip-flop before the next update request and to lock the pass transistor to guarantce that the $\overline{W R T \text { TITIRU }}$ signal remains 


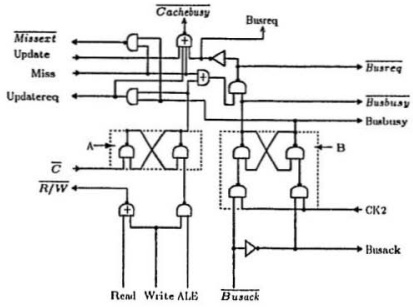

Figure 48: The Circuit for the Bus Control Signal Generator

unchanged until the end of $C K 1^{\prime \prime}$ even though the flip-flop is reset by $\overline{C K^{\prime \prime}}$. The operation of this circuit is shown in the timing diagram of Fig. 47 (c). Note that the WRTTETHRW signal becomes low at the beginning of $\mathrm{CKI}^{\prime}$ and returns to the high level at the end of $C K 1^{\prime \prime}$.

The bus control gencrator in Fig. 48 generates a numler of sigmals used for communication with the processor and system bus controller. In Fig. 18, $R$ and $W$ come from the processor and are used to access to the cache memory. $\overline{R / W}$ is sent to the bus uplate waicher to check the update request on the system bus. Only when both $\mathrm{I} V$ and $R$ are low is the signal $\overline{R / W}$ set high. When $A L E$ from the processor is asserted during a write operation, the $\mathrm{W}$ signal from the processor 
is latched into flip-flop $A$ (called the write flag). A request $\overline{B U S R E Q}$ for $11 \mathrm{~s}^{\circ}$ of the system bus can only be made by either a write operation (know: by lhe statte of the write fing) or a line iniss (indicated by the miss flag as long as Hip-flop $\mathrm{B}$, the bus busy flag, is reset). In the case of a line miss, $\overline{B U B B U S Y}$ is high. while BUSBUSY is low, so that $\overline{B U S R E Q}$ is low. The signal $\overline{B U S R E Q}$ is s. out to request the use of the system bus. Note that at this time BUSTIST is invalid (logical 1) since there is not a valid $\overline{B U S A C K}$ signal from the system bu. coniroller. After the cache sends the signal $\overline{B U S R E Q}$ to the systen bus controller. the bus controller responds to the cache request for the use of the system luts ly generating a low $\overline{B U S} \overline{A C K}$ signal to inform the cache that it call use He sistert bus as soon as the system bus arbiter makes a grant to this carthe. (Onre a watirl $\overline{B U S A C K}$ signal (low) from the bus controller is received by the cache during $(* k \cdot)$ the bus busy flag latches $\overline{B U S A C K}$ and in turn makes $\overline{B U S B U S Y}$ low to mply to the bus controller that the cache memory is using the system bus. Neww hilk $\overline{B U S B U S Y}$ also eliminates the bus request made before by pulling up $\overline{B ! S R l: Q}$. The high BUSBUSY signal can gate out the MISS signal from the mise llat? to form a new signal $\overline{M I S S E X T}$ and/or the signal from the write flag to transulit UPDATEREQ to the system bus. If only the miss flag is set, it means the rache memory is doing a miss operation caused by a read operation. If only the write. Hay is set, the cacle is doing a write-through operation for a write operation. If heth the miss flag and the write flag are set, the cache is working on a miss operation rautscil by a write operation. When the miss flag rises, the valid MISSEXT signal is se.II 


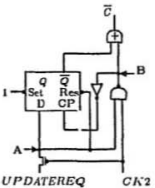

(a)

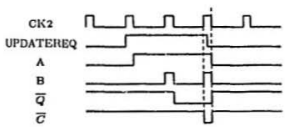

(b)

Figure 49: The Update Request Clear Circuit

valid $\overline{M I S S E X T}$ signal is sent out to inform the system bus to satisfy the miss operation during BUSBUSY; and when the write flag is held, the UPDATEREQ signal causes a $\overline{S E A R C I I N T}$ signal to ask the main memory and all other caches to update the data on the system data bus. Furthermore, there is another signal $\overline{C A C H E B U S Y}$ to be sent out to inform the processor to be idle under certain conditions. The conditions which make $\overline{C A C H E B U S Y}$ valid are the following:

1. to update other caches (UPDATEREQ),

2. to be updated by other caches (UPDATE),

3. to have a line-miss operation ( $/ I S S S)$,

4. to request the use of the system bus ( $\overline{B U S R E Q})$.

If the write flag is set by $W$, it has to be resel by $\bar{C}$ for next operation after a certain period, during which the cache does a write operation. 
The circuit in Fig, 19 (a) is employed to produce a clear signal $\bar{C}$ to reset the write flag. Initially the output $\bar{Q}$ of the falling edge-triggercd D fip-flop is at logical 1 to lock the 2-input OR-gatc so that the output $\bar{C}$ is high. If there is a high UPDATEREQ signal before the beginning of a pulse of $C K 2$ (note that UPDATEREQ can only begin to be high during CK2, sce Fig. 48), the UPDATEREQ signal remaius on the D input of the flip-flop until the end of the CK2 pulse even though the UPDATEREQ signal changes during the $C K 2$ pulse. The first pulse of $C K 2$ passes the 2-input NAND-gale to produce an inverted pulse on one input of the OR-gate while it is also imposed on the input $C P$ of the flip-llop. During this pulse, $\bar{C}$ is not clianged since $\bar{Q}$ locks the OR-gate. The falling edge of the first $C K 2$ pulse latches the high UP DAT EREQ signal into the flip-flop which makes $\bar{Q}$ switch from 1 to 0 . Thus, after the first $C K^{2} 2$ pulse, $\bar{Q}$ is low so that the 2-input OR-gate now becomes active. During the second pulse from $C K 2$, the inverted pulse from $\mathrm{CK}_{2}$ prsses the 2-input OR-gate to make the clear signal $\bar{C}$ valid while $C K 2$ is imposed on $C P$. The $\bar{C}$ signal resets the write fing to stop the UPDATEREQ signal. The UPDATEREQ signal is changed from 1 to $O$ to end the update request on the system bus. Note that the low UPDATEREQ signal is not reflected on the $D$ input of the flip-flop since it does not get through the pass transistor until the end of the second $C K 2$ pulse so that the $\bar{C}$ signal is complete. At the end of the $C K^{\prime} 2$ pulse, the llip-flop is reset by the low UP DAT E REQ signal to make $\bar{Q}$ logical 1 which locks the $\bar{C}$ signal again. The operation of this circuit is shown in the tining diagram of Fig. 49 (b). 


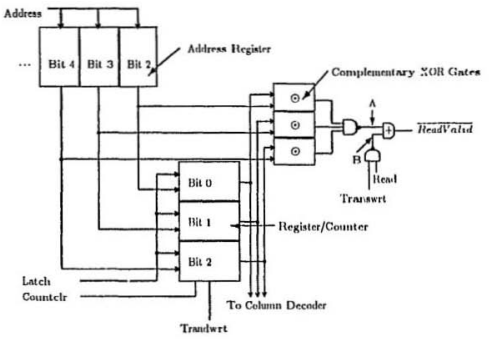

(a) The Circuit of Mead Valird
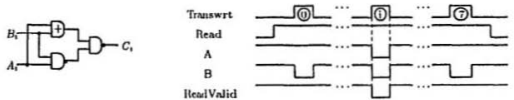

(b) The Complementary XNOR Gate

(c) The Tining Diagrain of The flead Valid

Figure 50: The Read Valid Circuil for Read Miss 
Fig. 50 (a) depicts a special circuit called read valid circuit which is used to create a signal READVALID to inform the processor that the data on the data bus are the data requested during transforring a missing line to the caclie. The processor can receive the data from the bus without reading the data from the cache memory after transfer of the missing linc. Thus the delay time for transferring a missing line for a read operation is decreased. During the transfer of a missing line, the counter/register operates as a counter. For each pulse of the TRANSFWRT signal from the transfer decomposer, the counter increases by 1 . The 3 bits of the counter are compared with the corresponding bits, bit 2 to bit 4 inclusive, of the address register simultancously. If all 3 pairs of the comparator ityputs are matched, the output of the 3-input NAND-gate is logical 0 at point $\mathrm{A}$. If the line miss is caused by a read operation (READ is high), the inverted TRANSFWRT signal arrives at point $B$. In this case, the $\overline{R E^{\prime} A D V A L I D}$ signal is valid (low level). This $\overline{R E A D V A L I D}$ is sent to the associated processor, and when the processor receives the READVALID, it reads the data on the data bus immediately. 'The comparator consists of three complementary XOR-gates. Fig. 50 (b) shows the logic circuit for one complemontary XOlR-gatc. The output becomes high only if two inputs of the circuit have the sane values. The operation of the read valid circuil is illustrated in lig. 50 (c).

In this chapter, the memory and control unil are designed, implemented and sitnulatecl. Eliminating both cache rewriting during a write miss and data bypass 10 processors during a read miss will reduce line-transfer time. 


\section{USE IN A MULTIPROCESSOR SYSTEM}

In a large modern computer system where there are ofuen several independent. processors with a shared memory, competition between intereomected processurs for access to the shared memory may become a serious problen since several of the high speed processing elements may try to reference the shared main momory at the. same time. The performance of such multiprocessor systems is limited hy thr sperd and bandwidth of the bus and the main memory. A key to cflicient operation is to reduce both network traffic and direct references to the main memors: 'The lenis memory reference latency caused by the network can be greatly reduced by the lowal memory for each processing element. since the majority of references to the main memory can be captured by a local memory such as a cache memury [?, I0]. lifig. in Illustrates a typical cache-based multiprocessor systen with a shared memory, in which each processor has an attached cache memory. Althought the usc of aiclurs in a multiprocessor system can greatly reduce the bus traffic and speed up, the system. such a system can cause a coherence problem because multiple coppies of that it in the shared main memory will likely reside in several different carde's all the simw" time.

\subsection{The Coherence Solution Strategy}

Since the use of multiple private cache memorics can cause a caclue colin renere problem, a reliable strategy must be found to kcep data in the system colierent. 


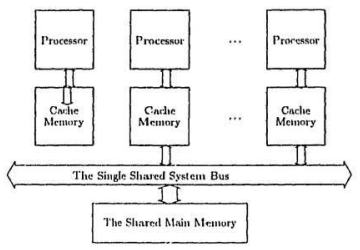

Figure 51: A Typical Cache-based Multiprocessor Systern

Many different solutions have been proposed for this problem $[5,8,10,12,14]$. $A$ menory system is coherent if the value returned from a read in the system reflects exactly the last value written in the referenced address by any processing element. There are two kinds of data incolerence [7]:

1. After the data in caches ate updated by the processing elements, they are not consistent with those in the main menory.

2. Multiple copics of a given line of data can exist in several caches; updating any copy of this line by a processing clement will cause the values in caches associated with other processing elements to be obsoletc. 
To eliminate the first case, a write-through policy is chosen in this system to keep the information between the main memory and caches consistent. Whenever there is a write requesh for a given address, the copies of the requested ditit in both the cache and the main momory are updated sinultancously with the new value. This scheme has sone advantages [5]: first, it can be implenented without complicated logic. Second, constant updating of both the cache and the main memory at every write request keeps the information in the main memory always consistent with that in the caches. llence, if there is a writc request for all aldress that is not in the cache, the systern can simply transer the requested line from the main memory to the cache to sntisfy the request of the processing elenent using a replacement policy without writing-back the old line before it is replaced with the new one since the data in main menory are always clean. Therefore, it is an effective way to handle this type of coherence problem in anulticache system with a shared main memory.

In the second case, an updaling algorithm is employed rather than incalidation. That is, whenever there is a write request to a cache from the processor, this request. will be broadeast to all the caches in the system to cause cach one to scarch for any copics of the requested data. If there are any, they are updated while the copy in the main memory is rewritten. Otherwise, nothing is done in the caches. The major drawback is that it does not tend to minimize communication network and main menory traffic caused by write operations and foress all bie caches to do update operations, even copics of the data to be updated do not reside in most of 
the caches.

In this system, the uritc-through policy and updating are combined as an algorithm write-through with updating to handle both coherence situations. Whenever there is a write rejuest from a processor, this request is broadcast to all the caches to inform the caches to update the data being written, if applicable; meanwhile, the main menory will receive the correct value for the data. The write-lhrough with updating is based on the expectation that, if the data are actively shared, the caches that have copies of updated data will use the copies before they are purged.

Data can be classificd as shared and unshared as well as readable and writable $[10,15]$. 'The clata are defined as shuret, including realable or writable variables, if they currently reside in more than one cache, while the term unshared datu means the data can only reside in one cache at any time. Therefore, there are four kinds of data:

1. Shared read/write data are the data which can be cither read or written by several processors at the same time, such as shared read/write variables.

2. Shared read-only data are those which can only be read by several processors, such as shared only-read variables and instructions (assuming that programs are not self-modifying).

3. Unshared read/write data, meaning the data can only be read or written by one proccssor at any time. 
4. Unshared only-read data are defined as those which can only reside in one cache at any time.

This policy is more appropriate for the case where much of the shared data (a number of caches share the sane data) is to be processed concurrently anong processors. When a processor rewrites the shared data in its cache, the copies in all other caches are updated immediately so that other associated processurs do not need to transfer the updated data from the main memory when they have to use the updated copies. An example to illustrate that this policy is efficient is managenent. of the common shared queue. It is assumed that this queuc with a semaphore cxists in each of several caches at the same time. When one of the processors intends to update the queue, it first checks the semaphore to see if the queue is being used by the other processor. If the queue is not used, the processor sets the semaphore in the corresponding cache. Meanwhile, this updated semaphore is broadcast to update the semnphore in all the caches, to prevent the queue from being used by any other caches at this time. After updating the queue, the processor resels the semaphore. The reset semaphore is also broadcast to update those copics in other caches. If the queue is used by any of other caches, the processor must wait until the semaphore in its cache is reset. Another example is the calculation of the sum of products of two sequences of numbers: $S U M=A_{1} B_{1}+A_{2} B_{2}+\cdots+A_{N} B_{N}$, and the corresponding program is as follows: 


$$
\begin{aligned}
& S U M:=0 \\
& \text { for } i=: 1 \text { to } N \text { do } \\
& S U M:=S U M+A(i) * B(i) ;
\end{aligned}
$$

Assume that there are $N$ processors for this calculation, the variable $S U M$ is shared and there is a semaphore initialized. To execute this program concurrently with $N$ processors, first, all the processors compule products of two numbers (that is, processor $i$ calculates $A(i) * B(i), 1 \leq i \leq N)$, and the results are stored in their corresponding local variables. Then the intermediate results are added together by serialization. Any one of the processors intending to add its intermediate result into the global-shared variable $S U M$ must check the semaphore to see if it has been set by another processor. If so, the processor must wait until SUM is released by the operating processor by reseting the scmaphore. Otherwise, the processor sets the semaphore to prevent SUM from bcing updated by other requesting processors at this time, and then adds its local intermediate result into the SUM variable. The result of the addition is broadenst (caused by the write operation for the SUM variable) to all other processors, updating their copies of SUM. Then the processor resets the semiphore for the next sum operation to be done by one of the other requuesting processors. Finally, in all the caches, there are consistent copics of SUM 
which may be used for the next parallel calculations. Unlike this updating policy, the invalidating policy simply invalidates all the copies of SUM in other caches as the operating processor writes the partial result into the copy of SUM in its cache. Thus, when any other processors want to continue the following operation of the sum, they have to transfer the correct partial result of SUM from the main memory before doing the sum operation. Hence, in this case, the updating policy is more efficient than the invalidating policy.

For write-through with uplating, it scems that low miss ratios will increase the probability that each of the updated data in the caches can be used before they are purged since low miss ratios indicales fewer purges of cache lines. Therefore, a larger size and a higher set associativity of the cache are preferred for this policy . On the other hand, this policy incurs the cost of updating all the caches for each write operation, and only a few updated copies may be used by respective caches before the lines containing the copies are removed for requested lines. The worst case for this policy is that no updates are useful for other caches; this happens, for example, when all the processors execute independently their own processes without use of shared data.

\subsubsection{The Protocols between the Bus and the Cache}

In this cache system, there is a mechanison for the cache to communicate with the system bus; an asynchronous single system bus is assumed. Generally, the cache has to communicate with the system bus in three chese; the first is a write 


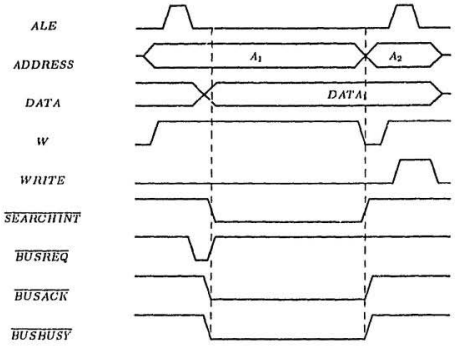

Figure 52: Communication between the Cache and Bus for a Write Oporation operation in which the cache has to send the data to be written onto the system bus to update both other caches and the main memory, the second is the transfer of a missing line in which the missing line is transferred to the cache via the system bus, and the third is an update request from another cache.

Fig. 52 shows a timing diagram for a write opcration. In the diagram, all the control signals are active low except those from the processor, like $W, W R I T E$, and $A L E$. When $W$ is asserted, the processor is doing a write operation on the cache, along with a valid address on the address bus of the cache. ALE latchs the address into the address register of the cache memory. After searching its directory, the cache system makes a bus request $\overline{B U S R E Q}$ for the use of the system bus to 
the system bus controller. Meanwhile, the cache checks SEARCHINT to see if there is an upclate request from any of other caches during BUSREQ. If the system bus grants the system bus to the cache via the bus arbiter, $\overline{B U S A C K}$ is set low which removes the request $\overline{B U S R E Q}$. Now the cache sends out the bus busy signal $\overline{B U S B U S Y}$, along with the address and data on the system bus, to reply to the bus controller that the bus is being used. Since this cache combines the wrilethrough policy and an uplating algorithm to simplify the control, it also signals an upclate request $\overline{S E A R C I I N T}$ onto the system bus to have all other caches do an update operation. After a two-cycle period, the cache removes the request $\overline{S E A R C H I N T}$, which make the bus controller invalidate $\overline{B U S A C K}$. Invalidation of $\overline{B U S A C K}$ clears the signal $\overline{B U S B U S Y}$, and the cache inforns the processor that the write operation has fitished, which will make the processor remove $W$ for next operation. As soon as the bus controller receives a $\overline{B U S B U S Y}$ signal, it selects one bus request from a bus request queue by sending a valid $\overline{B U S A C K}$ to the selected cache.

Communication between the cache and the system bus for a line miss is more complicated than that for a write operation. Fig. 12 illustrates the communication operation betwern the cache and the system bus for a line miss cansed by a write: operation. When the cache receives a write request from the processor, it makes a bus use request $\overline{B U S R E Q}$ to the bus controller since the datia do not reside in the cache. After the cache detects $\overline{B U S A C K}$, it removes $\overline{B U S R E Q}$ and semels out $\overline{B U S B U S Y}$ to the bus controller, requesting use of the system bus. Menuwhile. 


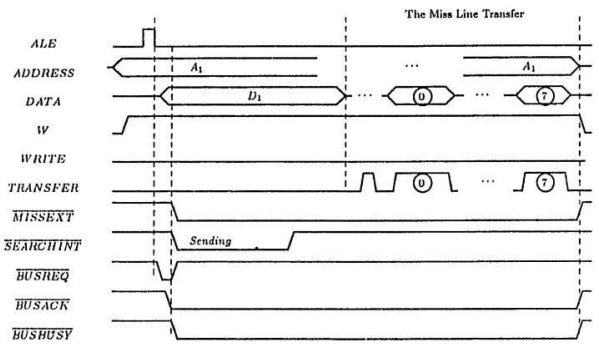

Figure 53: Communication between the Caclic and Bus for a Line Miss it also gates out the updating request $\overline{S E A R C H I N T}$ to all other caches and the main memory and the transfer request $\overline{M I S S E X T}$ to the main memory. After the update operation for a write request, the main memory issues the requested line, accompanicd by the TRANSFER signal, to the cache. As soon as the transfer operation is finished, the main memory informs the bus controller so that the bus arbiler clears $\overline{B U S A C K}$. The high $\overline{B U S A C K}$ signal in turn removes the requests $\overline{B U S B U S Y}$ and $\overline{M I S S E X T}$. The cache will inform the processor to terminate the write operation by making $\overline{C A C I E B U S Y}$ high. Note that there is no need to update the requested data in the cache menory since the line, being transferred from the main memory, contains that data updated. 


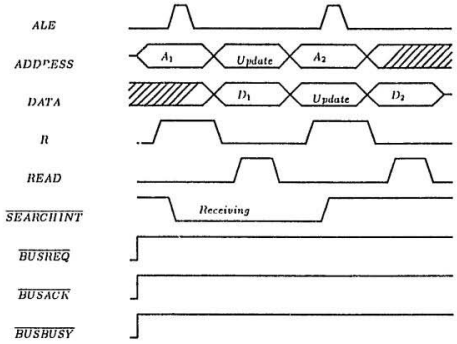

Figurc 51: Communication for an Update Operation

If a line miss is caused by a read request, no $\overline{S E A R C H I N T}$ signal is required, since no update operation is necessary. Only the line transfer operation is done, as shown in Fig. 53. Note that the shared main memory typically consists of interleaved modules so that a requested line can be transferred in a short timc.

Communication between the cache and the bus for an updatc operation is shown in Fig. 54. When the processor sends a read request $R$ to the cache, along with the requested address $A_{1}$ on the address bus, the caclie does the read opcration. After the read operation is finished (indicated by change of $R$ from 1 to 0 ), the cache allows the updating address on the system bus to reach the cache address bus and latches this address into the cache addless register for the directory search. 
At the same time, the cache reads out the data $D_{1}$ (Corresponding to $A_{1}$ ) during the first pulse of the READ signal, since the cache is pipclined. During this time, the cache informs the processor to wait for one cycle. In this cycle, the cache can determine if the data to be updated are in the cache or not, and at the end of the cycle the directory has finished and is ready for the next request. Therefore, in the next cycle, the processor sends the second read request, and the directory is searched for the second request while the cache memory unit is updating the data requested for the update operation on the cache data bus if the updating data reside in the cache. In the following cycle, the cache sends the data to satisfy the sccond read request of the processor. Since the cache does not intend to use the system for read operations, $\overline{B U S R E Q}, \overline{B U S A C K}$, as well as $\overline{B U S B U S Y}$ remain high during the update operation. Note that the address and the associated data for the update operation are placed onto the cache address bus and data bus from the system address bus and data bus, respectively, under the control of four signals which will be discussed in the following section.

\subsubsection{The Protocols between the Processor and the Cache}

In order to communicale with the processor in some special cases, the cache has a signal $\overline{C A C I I E B U S Y}$ to inform the processor, which is directly connected with the cache, to be idle. The conditions for a valid signal are: 
1. Occurrence of a line miss or an update request to other caclics.

2. Updating of the cache.

3. Waiting for use of the system bus (the system bus is being used by another cache).

Whenever any of these thrce conditions are true, $\overline{C A C / 16 / 3 V S Y}$ is valid, which makes the processor remain idle until $\overline{C A C H E B U S Y}$ is invalid. Ilow long the processor is idle depends on the particular condition; for example, updating the cache nocds at most two cyclcs.

Because of the limited unmber of pins on a chip, this system has only 32 pins for addresses and 32 pins for data. These pins are used by both the processor and the system bus. Therefore, the addresses both from the processor for access operations and from the bus for updating of the request data have to be lateled in the address register of the cache at different times. This is realized by two bi-directional switch arrays, BSAl and BSA2, as shown in Fig. 14. Each array has two parts, part 1 for the data bus and part 2 for the address bus. BSAl is uscd to control the path from the cache bus (both the address bus and data bus) to the processor; $\mathrm{BSA} 2$ controls the path from the cache bus to the systcm bus. The control siguals are from the caclie, and they are based on different conditions.

Usually BSA1 is on and BSA2 is off since most of the time the cache communicates with its processur. During a write operation to the cache, the data being written are broadeast on the bus to update other caches and rewrite the main 


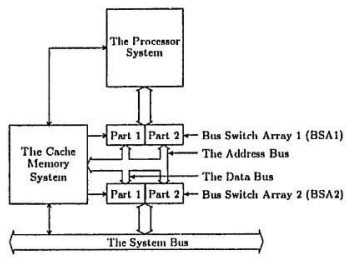

Figure 55: The Processor Subsystem

memory after the cache system receives the $B U^{\prime} S A C K$ signal from the bus conttroller, while SEARCIIINT is sent onto the bus to inform other caches and the main memory. BSA2 switches on at this time to gate the data and address to the system bus. $\Lambda$ fter the write operation, BSA2 returns to the off state. When there is an update request on the bus, the system has to do the update operation. BSA1 switches off to cut off the path to the processor while BSA2 lurns on to connect the path to the bus for the update operation. After updating, the arrays return to their original states.

If there is a line miss caused by a reacl operation and if the data is in the main memory, the requested line is transferred from the main memory to the 
cache, one word at a time by interleaving. In this case, BSA2 is on while BSAI is off. Furthermore, once the transferring word on the bus is the word needed by the processor, the signal READVALID is generated by the cache to inform the processor to take the data on the bus to satisfy the processor instead of reading the requested data from the cache after the full line has beco transferred. If the line miss is caused by a write operation and the cache is granted use of the bus, first BSA2 switches on while BSA1 remains on, to gate the data dircelly onto the bus. Both the other caches and the main menory are updated. Then BSAI switelies off to cut of the path to the processor so that the updated line is only transferred to the cache via BSA2, without updating the requested data in the caclic alter transfer. Thus the delny for transferring a new line during a line miss can be decreased for both read and write operations.

The four signals, $A D D B U S 1, A D D B U S^{\prime 2}, D A T A B U S^{\prime} 1$, and DATABUS', control operations of the BSA1 and BSA2 described above. T'he ADDBUS1 signal controls operations of the address bus of BSA1 while ADDBUS'2 determines op. erations of the address bus of BSA2. Also DATABUS1 is used to control the data bus of BSA1, and the DATABUS2 signal is used to control the data bus of $13 S \wedge 2$. The timing diagram for operation of the signals for communication betwcen the cache and the processor are shown in Figures 57,58 , and 59 in the next section. 


\subsection{External Interface}

To be uscd in a cache-based computer system, the caclie needs to interface to other components in the computer systcm, including the associated processor, system bus, wain menory, etc. This scetion contains a brief description of the cache $1 / 0$ signals and timing.

\subsubsection{The Interface Signals}

The cache's external interface has 80 signals as shown in Fig. 56. A summary of the pin functions is given below:

$A_{0}-A_{31}$, Address bus lines (input). During execution of the write/read operation, these inputs are the address from the associated processor via part two of the Bus Switch Array 1 (BSA1). During execution of an update operation, they contain the address from other caches in the nultiprocessor system through part two of the Bus Switch Array 2 (BSA2).

$D_{0}-D_{31}$, Data bus lincs (3-state, bidirectional). These signals provide the data path between the caclic and the processor as well as the system bus. The data bus can transuit and accet data using the dynanic bus sizing capabilities of the cache memory; the dynamic data size may be one, two, three or four bytes, depending on the data recuirenent. During exccution of the write/read operation, these inputs/outputs are the data from/to the associated processor via part 1 of 13SAI. During execution of an update operation, they contain the data to be updated by this cacle from other caches through part 1 of BSA2. 


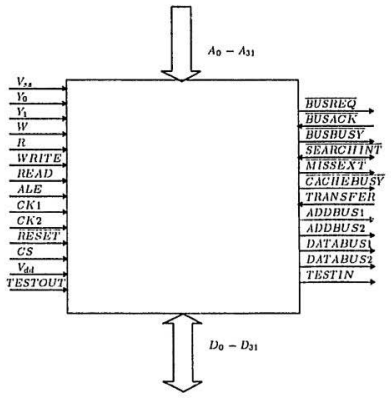

Figure 56: Pin Functions 
$Y_{0}, Y_{1}$, Accessed data size (input). These inputs from the processor indicate number of bytes of the data being accessed in one processor access cycle (See the previous section).

W, Write operation (input, active high). This signal indicates to the cache that the operation is a write operation.

$R$, Read operation (input, active high). It is used to indicalce a read operation.

Write, Write strobe (input, active high). This signal is uscd to write the dalat on the data bus into the cache. If the writc operation causes a line miss, this signal does not appear.

Read, Read strobe (input, active high). This signal is used to read the data requested by the processor fron the caclie. If the read operation causes a line miss, this signal does not appear.

$A L E$, Address latch enable (input, active high). It indicates that the address on the address bus is valid, and is used to latch the address into the address register of the cache.

CK1, Clock phrse 1 (input, active high). It is used to generate cache control signa's and pipcline the cache.

CK2, Clock phase 2 (input, active high). It is used to generate cache control signals and pipeline thic cachic.

CS, Chip selection (input, active high). This signal is used to indicate if this cache is selected cluring processor operations. It is very useful for nultiple cache chips used in a computer subsystem. 
$\overline{R E S E T}$, System reset (input, active low). It clears the internal logic of the cache memory.

$V_{D D}$, System power (input). It is a +5 volt power supply.

$V_{S s}$, System ground (input).

$\overline{B U S R E Q}$, Bus request (output, active low). This output is asserted to indicate that the cache requests use of the systen tus.

$\overline{B U S A C K}$, Bus grant acknowledge (input, active low). This signal indicates Lhat the system bus now is granted for use by the cache.

BUSBUSY, Bus busy (output, active low). This out put indicates to the system bus controller that the cache is using the system bus.

SEARCIIINT, Search interrupt (bidirectional, active low). If there is a write operation, this signal is an output which informs the main momory and other caches to update the data on the system bus. Otherwise, it is an input which is checked by the cache to see if there is an update request from other caches in the multiprocessor system.

MfISSEXT, Line miss (output, active low). It indicates that the dalia requested by the processor are not found in the cache and asks the main memory to transfer the missing line to the cactie.

TRANSF ER, Transfer of a missing line (inpul, active ligh). 'This signal from the main memory responds to the request, for transfer of a missing line to the cache. It is used to write the missing linc into the caclic.

$\overline{C A C H E ~ B U S Y, ~ C a c h c ~ b u s y ~(o u t p u t, ~ a c t i v e ~ l o w) . ~ T h i s ~ s i g n a l ~ i s ~ u s e d ~ t o ~ i n f o r m ~}$ 
the processor to be idle when it is valid.

ADDBUSI, Address bus control 1 (output, active high). This signal is used to control part 2 (for the address bus) of BSAI. BSA1 is employed to control the cache bus path to the processor (See the previous section).

$A D D B U S 2$, Address bus control 2 (output, active ligh). It is used to control part 2 (for the address bus) of BSA2. BSA2 is employed to coitrol the cache bus path to the system bus.

DATABUS1, Data bus control 1 (output, active high). 'This signal is used to control part 1 (for the data bus) of BSA1.

DATABUS2, Data bus control 2 (output, active high). This signal is used to control part 1 (for the data bus) of BSA2.

TESTIN, Test data input (input, active high). It is used to shift out the cacle memory addresses for testing only. 8 pulses are input for each address.

TESTOUT, Test data output (output, active high). It is used to shift out the caclie memory addresses for testing only. The outputs are an 8 -bit sequence of a cache line address from bit 0 to bit 7. After one testing pulse input from T'ESTIN, one bit of the cache line address can be observed on TESTOUT.

\subsubsection{The Timing Operations}

As described previously, operations of the cache can bc divided into five types: normal read, normal write, read-niss, write-miss, and update operations. The operations of these types can be depicted by Figures 57, 58, and 59. Fig. 57 shows 


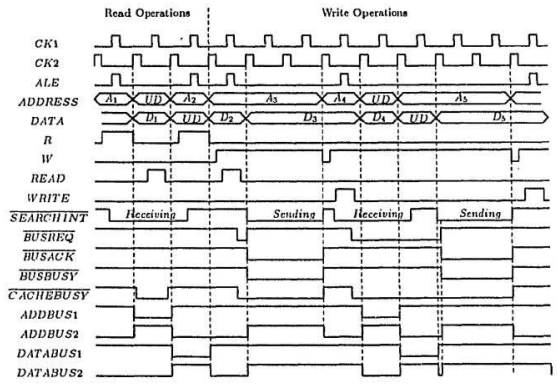

Figure 57: $\Lambda$ Tinting Diagram for Read/Write Operations 


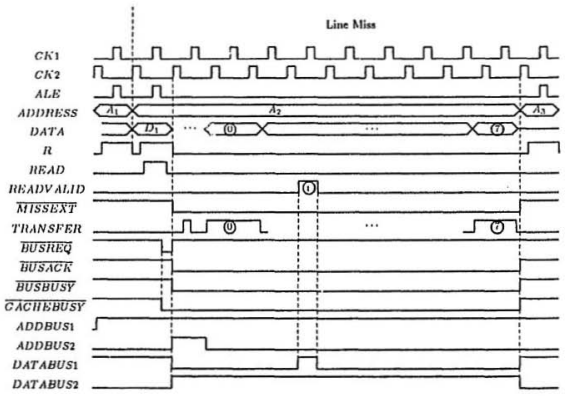

Figure 58: A Timing Diagram for Read Operations wilh a Line Miss 


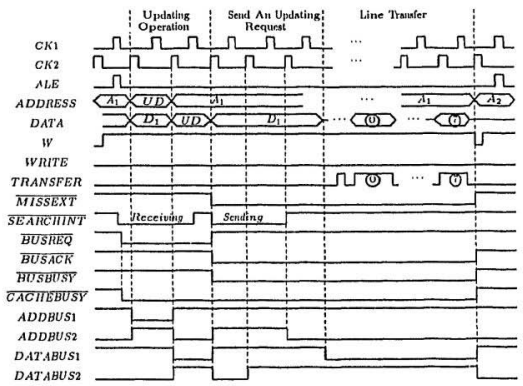

Figure 50: A Tinuing Diagram for Write Operations with a Line Miss 
how the normal read and write operations, including update operations requircd by other caches, are processed. The operations on the left hand side of the figure are the read operations with an update operation, while on the right hand side are the write opcrations with an update operation. We can see that the opcrations are pipelined from thie figure in which $\mathrm{A} 1, \mathrm{A2}$, etc. are an address sequence on the address bus while $\mathrm{D} 1, \mathrm{D} 2 \ldots$ are the corresponding data sejuence on the data bus. The valid period of the SEARCIINT signal labeled Receiving is is for the cadie to update the dat a after receiving an update recpuest from another caclie on SEARCIIINT, and the period labeled Sending is uscd to send an update regucst onto the SEARCIINT line. The address labeled UD is an address for updating and similarly the data labeled $U D$ are the data being updated. Fig. 58 illustrates the signal operations of the cache during a line miss caused by a read operation. The data labeled 0 to 7 on the data bus are eight words of the missing line obtained from the main menory by interleaving. Fig. 59 shows operations during a line miss caused by a write operation. In the figure, the period labeled Updating Operation is updating the cache during the request for the use of the system bus caused by a. line miss. The period labeled Send An Updating Request is used to send the data to be written and the corresponding address on to the systen bus for updating other caches and rewriting the main memory. The period labeled Line Transfer is transferring the miss line from the main memory into the cache. 


\subsection{Consideration of the System Bus and Main Memory}

A typical multiprocessor system usually consists of a set of processors and of a set of memory and $1 / 0$ modules linked together by means of an interconnection network. Information exchinge between either the processors themselves or the processors and shared main memory is accomplished by the interconnection network. Therefore, the interconnection network is a very inuortant part of the system. No generally accepted standard for an interconnection network exis ts, and since the interconnection network costs are a significant part of the system cost, the interconnection network is normally designed according to the requirements of the specific application. Ilere the Bus-Oriented network (the system bus) is discussed.

There are several typical implenentation policies for the single-bus arbiter. One implementation of the $\mathrm{N}$-user 1-server bus arbiter is based on a first-request firstservice policy. In this way, the bus arbiter always serves the request which was naate the longest thime ago anong the bus reçuests. In the cetse that there is more than one request being made at the sane time, the arbiler satisfies the one uade by the processor whose logical number is smallest. Fig. 60 depicts a block clingrann of the bus arbiter. It mainly consists of $N$ circuit blocks, each of which corresponds to a cache, and a state storage block. Signals $R_{0}-R_{N-1}$ are the bus requests from $N$ different caches for use of the system bus. For any block $i$, there is a signal $\overline{B u s A c k_{i}}$ informing the corresponding cache that it may use the bus. Validation of $\overline{B u s A c k_{i}}$ depends on the request $h_{i}$, the request grant $G_{i}$ fron the storage, and $C_{i-1}$. All the circuit blocks are connected in a daisy chain by $C_{0}$ to $C_{N-1}$ so that 


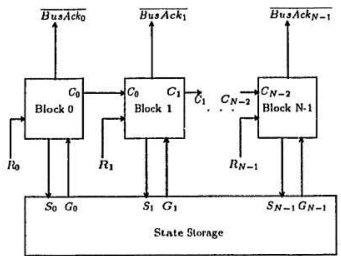

Figure 60: The N-User 1-Server Bus Arbiter

block $i$ can be invoked if and only if block 0 to block $i-1$ are not invoked by bus requests, and the $C_{i}$ will lock the following blocks (block $i+1$ to block $N-1$ ) not to be invoked. Therefore, none of the succeeding requests can be responded by the bus arbite: at this time. After the system bus is released by that served request, the next request will be granted use of the system bus under the same strategy. The state storage holds the information about the time the requests are made. Whenever the system bus scrves a request, the storage selects the one which is made the longest time ago by asserting $G_{i}$. After a request is served, the block $i$ will be reset and the state storage updated for next service.

ln general, devices in a multiprocessor system have different priorities for usc of the system bus. They can be grouped in terms of their priorities. The scheme as shown in Fig. 61 can be used for the arbitration unit in which there is a two-level 


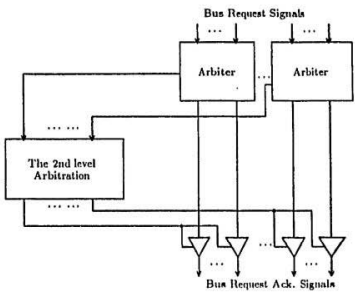

Figure 61: 'The Arbitration Unit

parallel bus arbitration. The first level is organized with arbiters shown in Pig. 60.

For cach group of requests with the same priority, an arbiter can be employed to select one request. The $C I I A I N$ signal lines from all arbiters are connecled and the second-level arbitration selects the highest priority arbiter using a daisy chain.

As indicated previously, the main menory can be divided into modules which are connected to the system bus. It is assuncd that the shared main memory for the system under consideration is partitioned into eight modules as shown in Fig. 62. The data bandwirlth of each module is one word (32 bits). The memory is organized in such a way that 8 words of a line are stored in 8 modules, respectively. That is, the first word of line $i$ resides in the module 0 while the second word of line $i$ resides in the module 1 and the third is in the module 2 , and so on. Ilence, a line can be transferred casily by interleaving. When there is a request for transfer 
of a missing line, each module sends a word in that line and the system bus delivers all of them by interleaving so that the delay is reduced.

Also for each module there is a buffer queuc for write requests. Thus, the specificd cathe only needs a short time to send the write request (including the data to be written and the corresponding address) to the given module without waiting for the main menory to complete the request. Whenever there is a write request entering one modulc, the module controller first checks to see if there is a write request in the buffer queue which is accessing to the same location as the cntering request. If so, the data of the request already waiting in the queue will be replaced by that of the entering request, and the entering request removed. Otherwise, the entering request is inserted at the end of the quene. Thus, the turite - vrite competition is eliminated in the memory module. When there is a cache miss, a line transfer is required, and all the modules transfer the missing line immediately without inserting the request in the queues. In the case that the line miss is caused by a write operation, first the module being overwritten checks the queue to see if there is a request in the queue for the same location. If so, the request in the queue is removed. Otherwise, the queue is unchanged. Then the module serves that write request causing a line miss by updating the requested menory location with the data on the system bus; the updated word is scul to the requesting cache with the other 7 words from respective modules by interleaving. Meanwhile, the other 7 modules serve the transfor request as they do for a transfer request caused by a read operation. When the line miss is caused by 


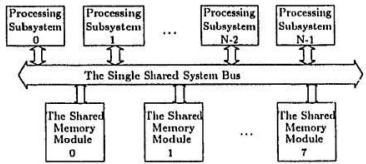

Figure 62: The Shared Main Memory Partitioned into 8 Modules

a read operation, each module checks its buffer queuc to sec if there is a request in the queue, for a write into the location to which the transfer request will access. If there is such a request, it is removed from the queue; then it is served immedialely. Thus, the so-called read -- write memory competition is handled. The module then sends the requested word onto the system bus. All 8 words from different modules are sent on the bus by interleaving. 'This can be done by the bus controller. Note that there is no read - read memory competition since the main memory only serves read operations during a missing line transfer and the single system bus only serves one request at a time.

\subsection{Simulations of the Cache-based Multiprocessor}

In previous sections, functions and structures of the cache menory and a multiprocessor environment were described, based on a writc-through with updaling cache 
colierence protocol, in which the multiple caches are used. In this section, a typical simulation model, as shown in Fig. 62, will be used to study the efficiency of such a shared-memory multiprocessor system. That is, we would like to determine how many processors can be used in the system without reaching saturation of this systcm. For simplification, a single bus is employed as the interconnection network between multiple caches and the shared mernory, although using a more complex interconnoction network such as a multi-bus system makes the system more efficicnt. Furthermore, in the model all the processors arc identical and ench processor has a private cache.

Thie model consists of a process for each processor, a process for each cache, and a process for the single bus. Bach processor generates a memory reference sequence to the associated cache. Memory reference streans in the system are produced with a specificd write operation ratio and a given cache miss ratio in the steady state. Writc operations are produced at random with a given read/write ratio. Each cache is implemented as has been described. For each processing subsystem, if the reference is a read operation and the requested line is present in the cache, the cache spends one cache cycle and the processor continucs. If the reference is a write operation and the requested line is found in the cache, the cache puts an update request into the service queue of the system bus, and spends two cycles to update all the caches and the main memory vin the single bus as soon as the update recuest is acknowledged by the hus. It is assumed that there is a buffer queuc in each memory module for write requests so that a write request can be sent to an 
appropriate memory module in two cache cycles, otherwise, a miss occurs. In this case, the cache needs to insert the transfer request into the service queue of the system bus, and the bus takes 11 cycles to transfer a requested line into the cache when the request is served by the bus. The time required to transfer a missing line is based on an assumption that a main memory cycle time is four cache cycles and the main memory transfers the missing line by interleaving, one cache cycle per word. So the transfer of a missing line requires one main memory cycle time (4 cache cycles) for the first word plus one cache cycle for each additional word ( 7 cache cycles). When the cache receives an update reguest from the bus, if the requested data are found, the cache spends two cache cycles. Otherwise, it only takes one cycle to search the directory. Note that hose caclics, waiting in the bus service queue for use of the system bus, inust halt until they arc releascrl from the bus queue after service. The bus process receives service requests from all caches and serves them in first-in first-out order, implemented by a first-in first out service queue in the simulation model. For one request in the quene, there are four items: Cache Number from which the request is made, Writc/Miss, Address, and Cachr Cycles to be used by the system bus. In this model, it is assumed that there is no delay when the bus services a requesh.

Fig. 63 summarizes the results of simulation for evaluation of the multiprocessor system wilh the proposed caches as private caches. Simulation ontputs include bus utilization figures and ollher paranelers under which the simulations ale run. The system power is defined as total sum of the processor utilization in the nutti- 
processor system, multiplied by 100 , and processor utilization is measured by the ratio of time spent doing useful work in a processor to the total runing time. In each figure, the simulation results obtained with the indicated parameter values are shown with from one to fifteen processors. The parameter Cycles gives the cache cycles executed during simulation. Fig. 63 (a) shows the simulations with an overall cache uniss ratio of 0.05 while Fig. 63 (b) gives the simulation results with an overall miss ratio of 0.03 . In each figure, there are five curves, each of which indicates a sinulation with write operation ratios varying from 10 percent to 30 percent for menory references. The system power rises until the system bus begins to reach saturation. When the bus utilization approaches 100 percont, the system power levels out. For each figmre, it is scen that the system power becones higher and the minimunn number of processors at which the single system bus reaches saturation increases as the write opcration ratio decreases. Comparison of the two figures indicates that a decrease in the overall miss ratio of the caches increases system power.

Although use of the specified caches in this given multiprocessor structure can greatly reduce references to the slow main memory, the single bus systen seems to be a botlleneck in the multiprocessor since write operations and transfer of miss lines under the write through protocol still make the bus busy. In order to further improve the system performance, an increase in system power and bus ability can be achieved as follows:

First, data can be labeled as either private or shared. If there is a write operation 


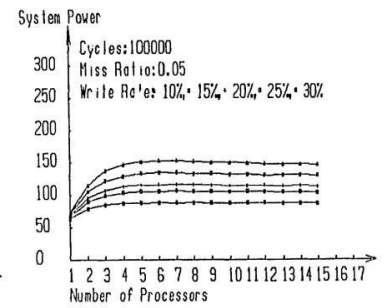

(a)

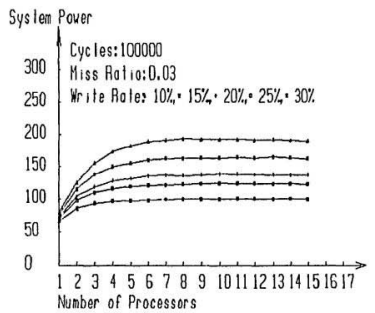

(b)

Figure 63: Simulations for the Multiprocessor System 
on private data, the cache does not issue an update request to all other caches while it sends an overwrite request to the main memory via the system bus. In this case, other caches can do useful work without bcing intercepted for an update operation. Thus, the system power would be increased although the system bus has the same traffic as that without this enforcenent. Note that in this scheme, there are two ignals requircd, instead of $\overline{S E A R C I I I N T}$, one is used to inform all caclies to update copies of the shared data and the other is used to request the main memory for a write operation.

Sccond, usc of caches with larger sizes can increase the system performance, since a lower overall utiss ratio for the caches results in both a higher system power and a higher bus utilization from the simulations. Also, the larger cache size makes the cache have a lower miss ratio. The multiprocessor system can take advantage of the efficient caches since a large size cache memory for each processor can easily be formed by using several cache chips selected by chip selects without decreasing the cache speed.

Third, the use of multiple busses would significantly increase system performance, because the wailing time of each cache for use of the system bus would certainly be decreased.

\subsection{Testing the Cache Memory}

Testing the circuit is the final step of a VLSI design, to determine if the circuit being lested has both correch logic and circuit operations. Although testing will be 
done only after fabrication, how to complete the lesting task has to be considered during circuit and architecture design.

Testing of the cache memory can be done using a microcomputer. All the I/O lines of the cache memory chip to be tested are connected to the microcomputer via an interface. All the signals for controlling operations of the cache menory and addresses and corresponding data are sent to the tested cache and all the corresponding results are received by the microcomputer. The microcomputer will check to see if the cache operations are correct. The microcomputer, step by step, will test all the functions of the cache memory.

In order to test this cache memory chip, there is a simple additional logic block: in the chip to shift out the cache memory address from the memory register. Therefore, for each main memory address referenced, the corresponding cache menory address and its content can be observed. Thus, we can create a table containing the main memory addresses for testing, the corresponding cache memory addresses, and the corresponding data during testing.

Fig. 64 illustrates the test circuit which can shift out the cache menory at. dresses. The 8 bit cache addresses are imposed on bits $\overline{B I T_{0}}-\overline{B I T}$ and the unt. tiplex is controled by a 3-bit counter. Whenever there is a pulse at TEST - IN, the counter is increased by 1 . One of the 8 bits of the cache address palsses the multiplex to the output TEST - OUT'. 'Thus, for shift-ont of a cache address, 8 pulses are imposed on the TEST - IN and 8 lits of the address can be receivet at TEST - OUT' one by one. 


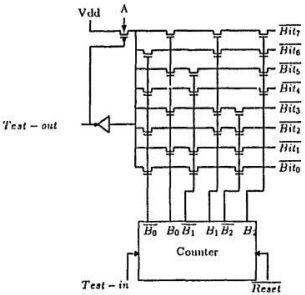

Figure 64: The Testing Circuit for Shifting-out Cache Line Addresses

In this chapter, use of the cache in a multiprocessor environment is described in which a system bus and a shared main memory are assumed. $\Lambda$ write-through with updating stratcgy is proposed and cmployed to kecp data in the system coherent. The system bus and shared memory structures are discussed. A queucing model is created and the system simulations have been done to evaluate the system performance. 


\section{CONCLUSIONS}

This cache meinory system has been laid out within a chip, using the 3 micron NTCMOS3 technology, and simulated. It has an $8 \mathrm{~K}$-byte coche monory ( 4 bytes for each word, 8 words for ench line), and it is organized as an 8-wny sel-associntive: cache. The cache memory is directly accessable to one, two, three, or four byles (onc: word) once by the associated processor. A two-phase clock is used to synchronize and pipeline the system. The clock period is 40 nanoseconds.

In the directory, there are 32 sets, therefore 8 line slots for ench set can be simultaneously compared. The address translation can be finished in 18 nanoseconds. Thus, the cache can safely trun out a result in 20 nanoseconds during read operations without line misses.

The least recently used line replacement strategy is enployed in the replacemeit. unit. There are 32 components, each one implemented by a bit matrix corresponid. ing to a set.

This cache memory can be used in a multiple processor system to improve the system performance; a write-through with updating policy, combination of a write-through and a uptating algorithm, is cmployed to kecp the information in the main menory consistent with that of the caches and to make the multicaches in the system coherent. The hit ratios of this cache memory, in terms of the Cinclie: Design Target Miss Ratios Table, are predicted to be over 95, percent.

Compared with on-chip caclie memory, this rache menory chip has a larger 
cache size and a low miss ratio. Unlike a cache system consisting of a cache controller and RAM chips, it is more flexible to build a cache system which has a larger cache capacity (more than $8 \mathrm{~K}$ bytes) for one processing element with several of the proposed cache memory chips by using the chip select signal; this docs not decreases the system specd. It is also easy to implement a cache system with separate cache memories for data and instructions. This multi-chip cache system also eliminates delay time caused by wire connections between the cache controller and $R \wedge M$ chips (off-chip delay).

Although this cache has many advantages, there are several drawbacks, due to linitations of the VLSl techuology used. It does not have a "snoop" directory which can be used to snoup the system bus for update operations, and in turn to eliminate the directory search time for updates. It dors not further reduce the references to the main memory caused by write operations, which can make heavy interconnection network traffic under the write-thwugh policy, especially in a single-bus sharcd-memory multiprocessor system.

An implementation using a more modern process technology, say a 1.5 micron technology, would permit a larger cache menory chip, or rather a large on-chip cache memory, with dual-directories and faster address translation. Also it could allow the inplementation of both the write-through and writc-back policies in a cache memory, which could make the cache have a great performance improvenent.

l'or higher performance, assuming the data are classified into shared and unsharrd as mentioned before, if there is a request for writing a shared read/write 
variable, the write-through policy is used to keep the multicaches and main menory consistent since the shared read/write variable may be modified by several proces. sors. If the accessed data are an unshared read/write variable, the write-back policy is employed to decrease the network traffic sinec only one valid copy of this uriable can exist in one cache. In the case that the line containing the unshared read/write variables is to be replaced by a new requested one at a carlie miss, if this line has been updated since it existed in the cache, it is written-back to the main menory before transfer of the new line to make information in the main menory correct. If it has been unchanged since it resided in the cache, it, like the line which contains the read-only data (including the shared and unshared reat-only variables as well as instructions), is simply overwritten by the new requested line, since it is consistent with that in the main memory. 


\section{References}

[1] Derek De Solla Price, "A Ilistory of Calculating Machines", IEEE Micro, Vol. 4, No. 1, 1984 pp. 24-36

[2] Harold S. Stone, Migh-performance Computer Architecture, Addison-Wesley, Reading, Mass., 1987

[3] R. E. Matick, Compuler Storage Systems and Technology, Jolın Wiley \& Sons, New York, 1977

[4] 'T. Kohonen, Content-Addressable Memorics, Springer-Verlag, Berlin, 1980

[5] A. V. Pohm and O. P. Agrawal, High-speed Memory Systems, Prentice-Hall, Reston, Virginia, 1983

[6] J. Erller, et al., "Issues Related to MIMI) Shared-memory Computer: the NYU Ultracomputer Approach", The 13th Ann. Int'l Symp. on Computer Archilecture, 1986, pp. 127-135

[i] W. C. Yen, D. W. L. Yen, and K. S. Fu, "Data Coherence Problem in a Multicache System", IEEE Tirans. on Computers, Vol. C-34, 1985, pp. 56-65

[8] S. Frank and A. lnsolberg, "Synapse Tightly Coupled Multiprocessors: a New Approach to Solve Old Problems", AFIPS Conf. Proc., Vol, 53, 1984, pp. 41.50 
[9] A. J. Smith, "Cache Memories", ACM Computing Surveys, Vol. 14, No. 3, 1982, pp. 473-528

[10] A. Gottlieb, et al., "The Ultracomputer - Designing a MIMD, Shared Mem. ory Parallel Machine", The 9th Ann. Int" Conf. on Computer Archilecture, 1982 , pp. $27-42$

[11] A. J. Smith, "Line (Block) Size Choice for CPU Cache Menory", ItKE Trans. on Computers, Vol, C-36, No. 9, 1987, p1). 1063-1074

[12] R. II. Katz, et al., "Implementing a Cache Consistency Protocol", The 12th Ann. Int'l Symp. on Computer Architecture, 1985, pp. 276-283

[13] P. Sweazey and A. J. Sinith, "A Class Of Compatible Cache Consistency Protocols and their Support by the IEEE Futurebus", The 12th Ann. Int't Symp. on Computer Architcciure, 1985, pp. 415-423

[14, ․ R. Goodman, "Using Cache Memory to Reduce Processor-memory 'Trallic", 10th Ann. Int' Symp. on Computer Architecture, 1983, pp. 124-131

[15] P. Bitar and A. M. Despain, "Multiprocessor Cache Synchronization ... Issues, Innovations, Evolution", The 12th Ann. Int'l Symp. on Computer Architecture, 1985, pp. $425-433$

[16] D. R. Cheriton, G. A. Slavenburg, and P. D. Boyle, "Sofl-controlled Caches in the VMP Multiprocessor", The ITh Ann. Int'l Symp. on Computer Amhitecture, 1985, pp. 366-374 
[17] M. Dubois, C. Scheurich, and F. Briggs, "Memory Access Buffering in Multiprocessors", The 12th Ann. Int'l Symp. on Computer Architecture, 1985, pp. $434-443$

[18] M. D. Mill, "A Case for Direcl-Mapped Caches", Computer, Dec., 1988, pp. 25-40

[19] M. Dubois and F. A. Briggs, "Eifects of Cache Coherency in Multiporocessors", IEEE Trans, on Computcrs, Vol. C-31, No. 11, 1982, pp. 1083-1099

[20] N. II. E. Weste, K. Principles of CMIOS VLSI Design, Addison-wesley, Reading, Mass., 1985

[21] B. Randell and P. C. Treleaven, VLSI Architeclure, Prentice-llall International, London, 1983

[22] F. J. Ilill and G. R. Peterson, Digital Systems, John Wilcy \& Son, Toronto, 1987

[23] M. A. Marsan, G. Balbo, and G. Conte, Performance Models of Mulliprocessor Systems, The MIT' Press, Cambridge, Mass, 1986

[24] X. Luo, and P. Gillard, "An Efficient Cache Menory Managenent Unit", Proc. of APICS Computcr Śsience Confcrence, 1988, pp. 1-12

[25] I1. J. Mitchell, 32-Bit Microprocessors, Williarn Collins Sons \& Co. Ltd, London, UK, 1986 
[26] M. Stansberry, "Cache Menory Design in 32-bil Microprocessor Systems", VLSI Systems Design, 1988, pp. 32-42

[27] B. Merril, "370/168 Cache Memory Performance", Share Computer Mleasurement and Evaluntion Netusletter, No. 26, 1974, pp. 98-101

[28] D. Phillips, "The Z80000 Microprocessor", IEEE Micro, Vol. 5, No. 6, 1985, pp. 23-36

[29] D. Alpert, et al., "32-Lit Processor Chip Integrates Major System Functions", Electronics, July, 1983, pp. 113-119

[30] R. Gregory, "Caching Designs Eliminate IVait States to Relieve Botulenecks", Computer Design, Oct., 1988, pp. 65-73

[31] D. MacGregor, D. Mothersole, and B. Moyer, "The Motorola MC68020", IEEE Micro, Vol. 4, No. 4, 1984, pp. 101-118

[32] H. Scales, P. Harrod, "The Design and Implementation of the MC68030 Cache Menories", IEEE Conf. On Computer Design, 1987, pp. 578-581

[33] Goldman, "First Look at Motorola's Latest 32-bit Processor", Electronics, Vol. 59, No. 31,1986 , pp. $71-75$

[34] D. W. Clark, "Cache Performance in VAX-11/780", ACM Trans. on Computter Systems, Vol. 1, No. 1, 1983, pp. 21-37 
[35] R. E. Matick, "Functional Cache Chip for Improved System Performance", IBM J. Res. Develop., Vol. 33, No. 1, 1989, pp 15-32

[36] P. Stenstrom, "Reducing Contention in Shared-Memory Multiprocessors", Computer, Vol. 21, No. 11, 1988, pp. 26-37

[37] S. Thakkar, P. Gifford, and G. Fielland, "The Balance Multiprocissor System", IEEE Micro, Vol. 8, No. 1, 1988, pp. 57-69

[38] J. Archibald and J. L. Baer, "Cache Coherence Protocols: Evaluation Using a Multiprocessor Simulation Modei", ACM Transactions on Computer Systems, Vol. 4, No. 4, 1980, pp. 273-298

[39] ^. Ayyad and B. Wilkinson, "Multiprocessor Sehene with Application to Macro-daLatlow", Alicroproccssors and Mictvsystems, Vol. 11, No. 5, 1987, pp. $255-263$

[40] J. K. Muppala and L. N. Bhuyan, "Arbiter Designs for Multiprocessor Interconnection Network", Microprocessing and Aficroproyramming, No. 26, 1989, pp. $31-43$ 




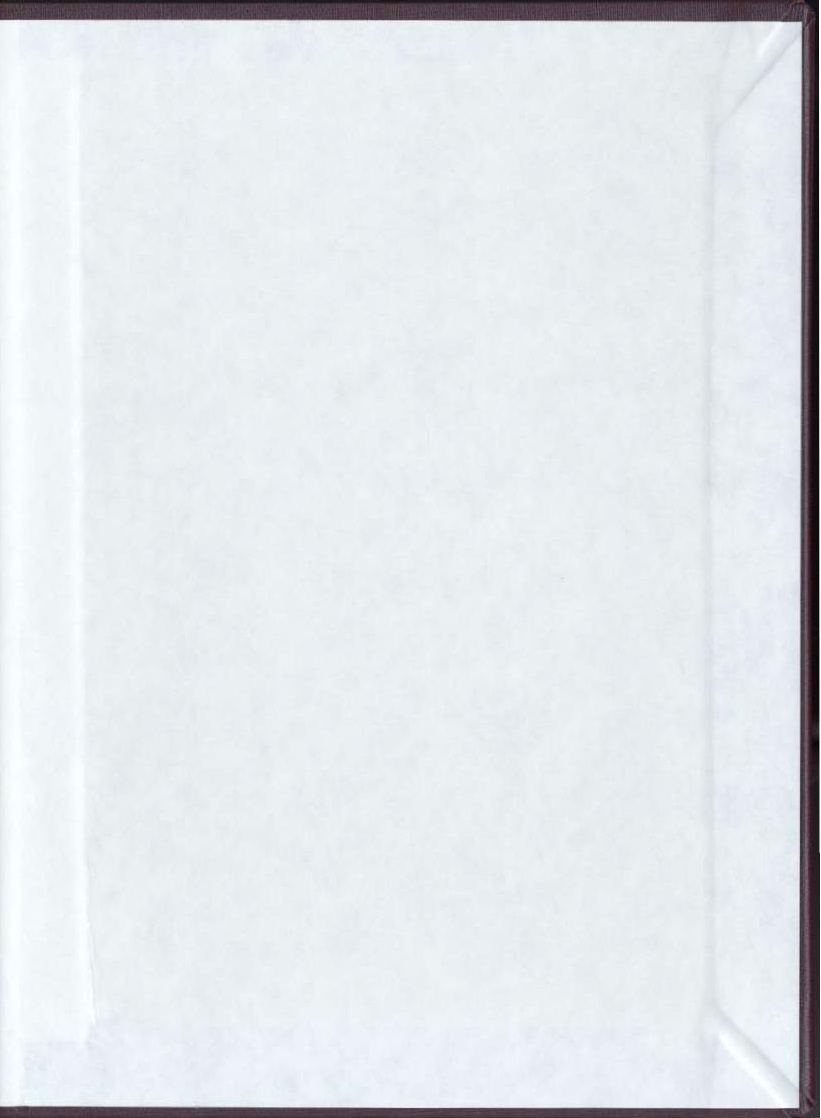


